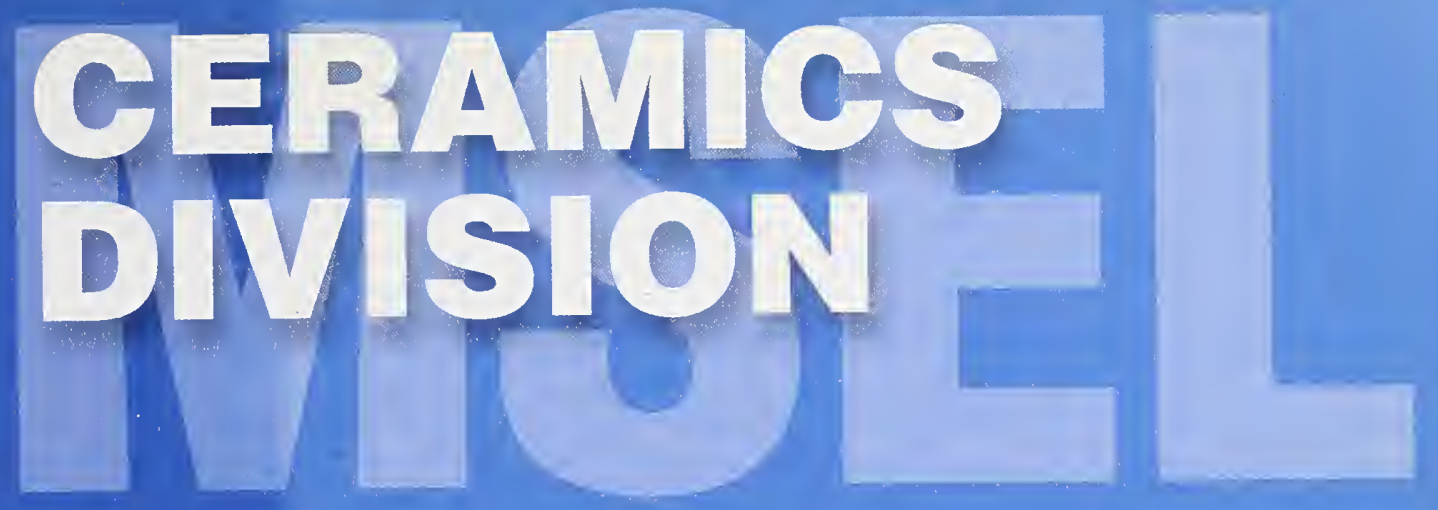

\title{
FY 2000 PROGRAMS AND ACCOMPLISHMENTS
}

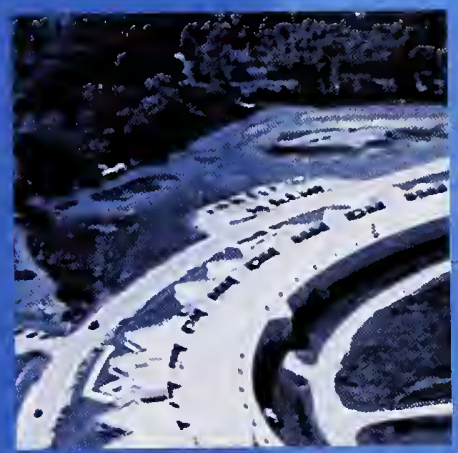

NGا

National Institute of Standards and Technology

Technology Administration

U. S. Department of Commerce NISTIR 6594

QC

100

.156

N0.6594

2001
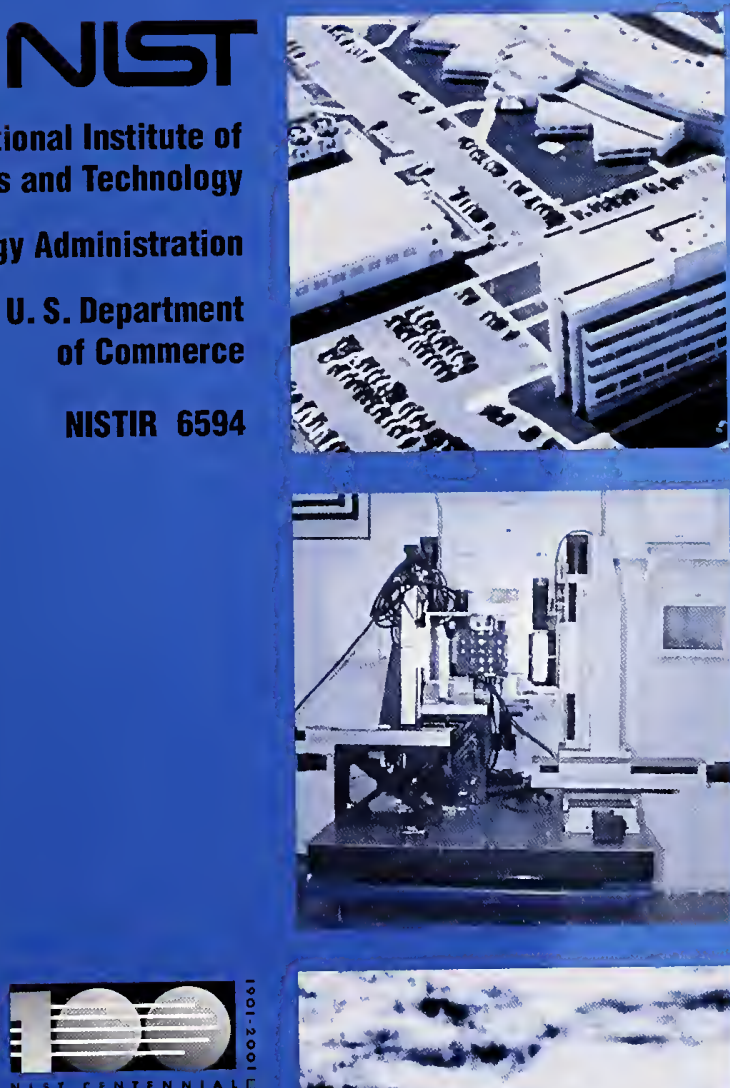
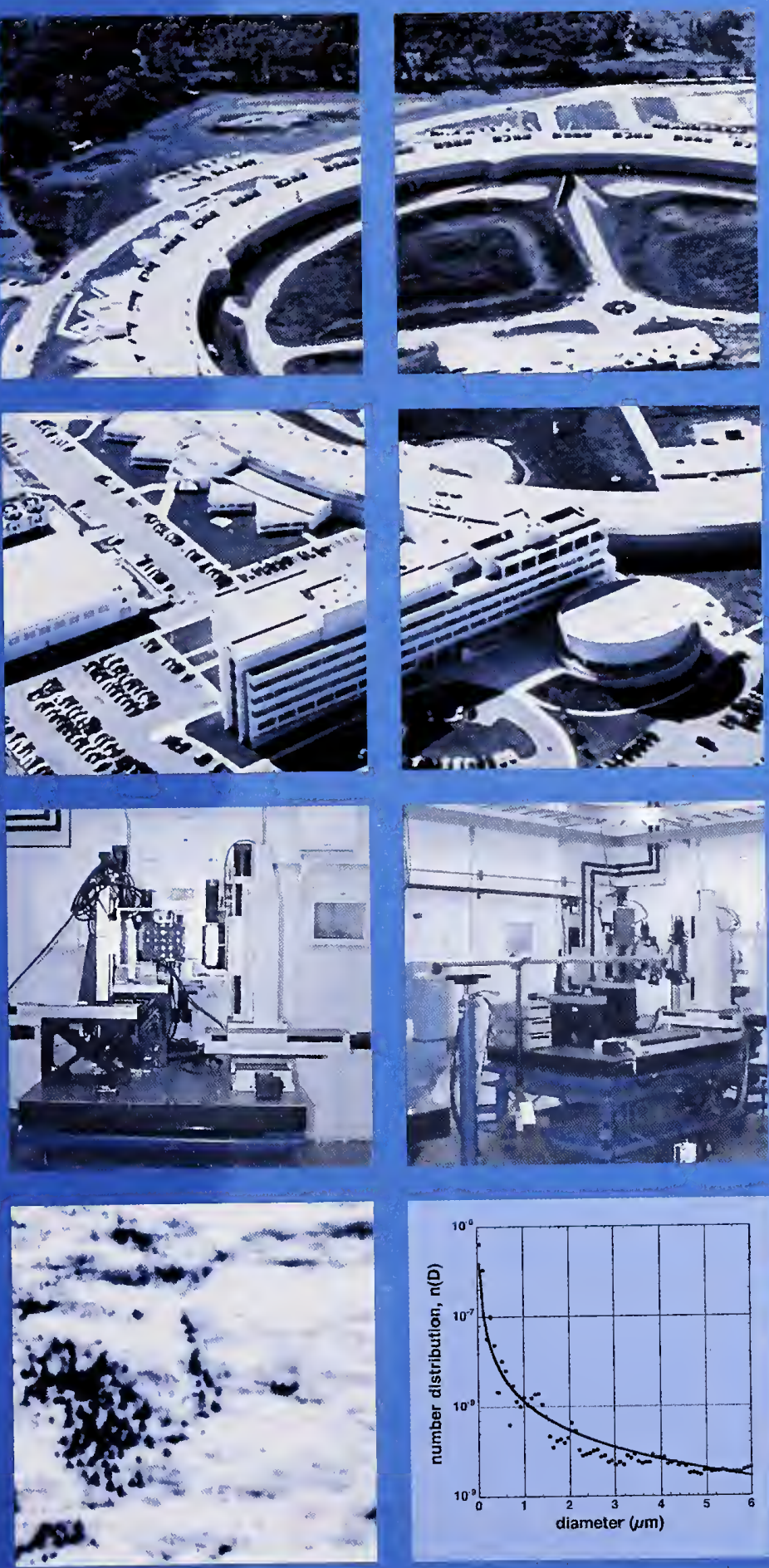
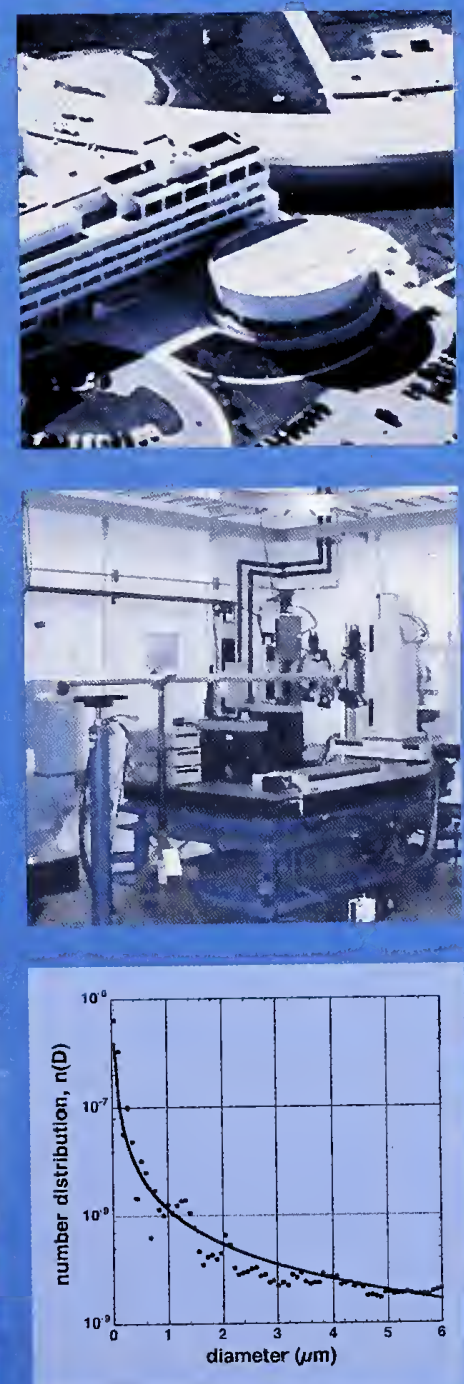
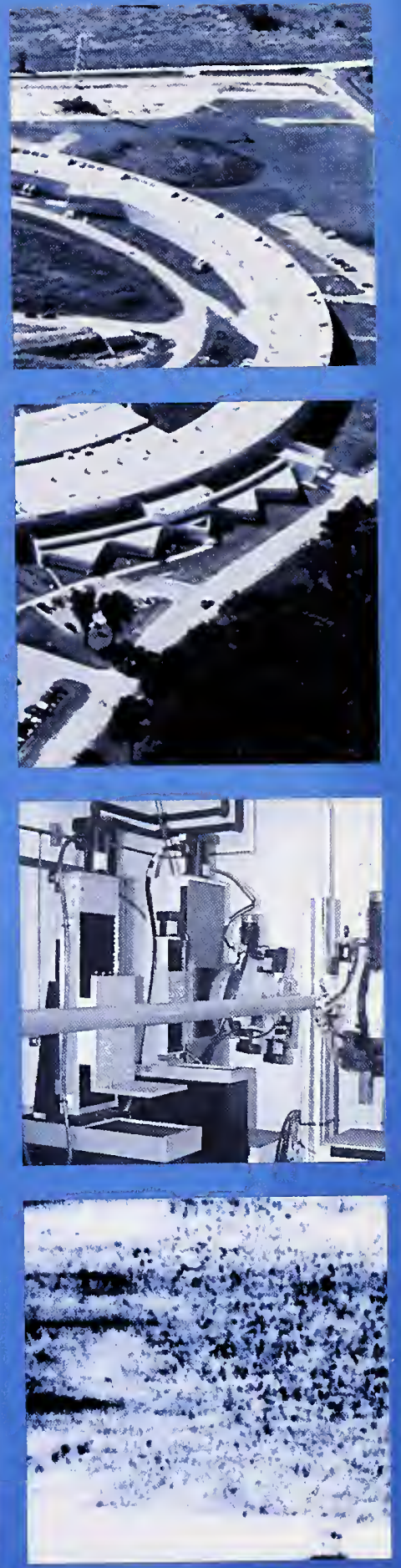


\section{Cover Caption}

Panels 1 - 6: Bird's eye view of the Advanced Photon Source (APS) at Argonne National Laboratory. NIST's Ceramics Division is part of a Collaborative Access Team at the APS known as UNI-CAT, which includes NIST, the University of Illinois, Oak Ridge National Laboratory, and UOP Research, Inc. UNI-CAT provides access to the nation's most intense X-ray beams for basic and applied research in materials science; biological science; physics; chemistry; environmental, geological; and innovative $X$-ray instrumentation.

Panels 7 - 9: The UNI-CAT ultra-small-angle X-ray scattering (USAXS) instrument.

Panel 10: USAXS image of mildly crept copper taken at a scattering vector, $Q$, of $1.3 \times 10^{-4} \AA^{-1}$. At this ultra-low scattering vector, large $(\sim 10 \mu \mathrm{m})$ cavities are seen.

Panel 11: Derived number distribution of creep cavities from statisticallysignificant USAXS data from the same mildly-crept copper sample shown in Panels 10 and 12, assuming spherical creep cavities.

Panel 12: USAXS image of the identical sample volume of mildly crept copper shown in Panel 10, but taken at scattering vector, $Q$, of $7.5 \times 10^{-4} \AA^{-1}$. At this larger scattering vector, smaller $(\sim 2 \mu \mathrm{m})$ cavities become visible. 
UNITED STATES

DEPARTMENT OF

COMMERCE

Donald L. Evans,

Secretary

\section{TECHNOLOGY}

ADMINISTRATION

Karen H. Brown,

Acting Under Secretary of

Commerce for Technology

NATIONAL

INSTITUTE OF

STANDARDS AND

TECHNOLOGY

Karen H. Brown,

Acting Director

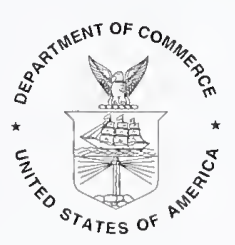

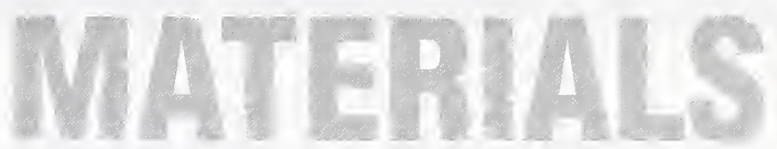
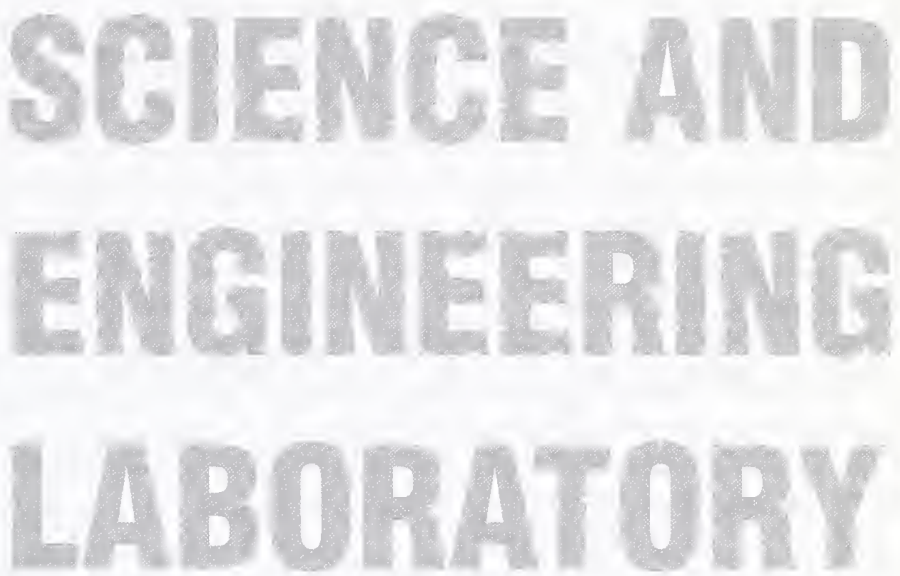

CERAMICS

FY 2000 PROGRAMS

AND

\section{ACCOMPLISHMENTS}

Stephen W. Freiman, Chief

Stanley J. Dapkunas, Deputy

NISTIR 6594

January 2001 


\section{Table of Contents}

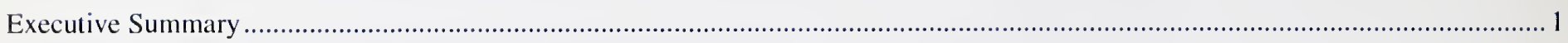

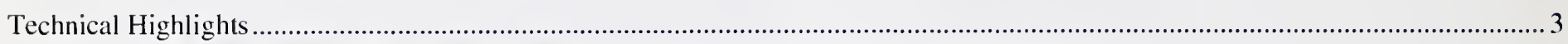

$\mathrm{AO}-\mathrm{Al}_{2} \mathrm{O}_{3}-\mathrm{Nb}_{2} \mathrm{O}_{5}(\mathrm{~A}=\mathrm{Ca}, \mathrm{Sr}, \mathrm{Ba})$ Phase Diagrams Reveal Potentially Useful Wireless Ceramic ....................................................... 4

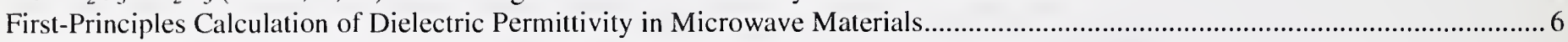

New Methodologies Characterize Machining Damage and Its Effect on Strength in Ceramics................................................... 8

NIST Recommended Practice Guide ................................................................................................................................ 10

Phase Equilibria of Ba-Nd-Cu-O, and Critical Single-Phase Region for Tailoring Superconducting

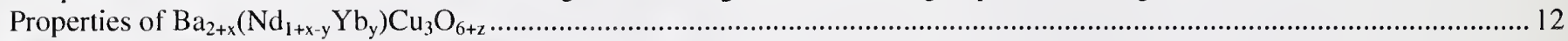

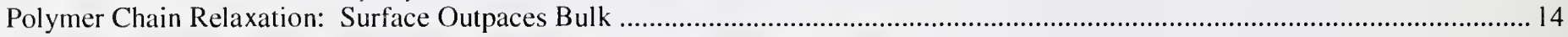

Spatially Resolved Valence Electronic Structure by X-Ray Standing Waves and

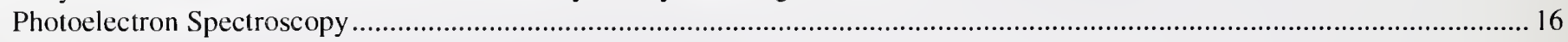

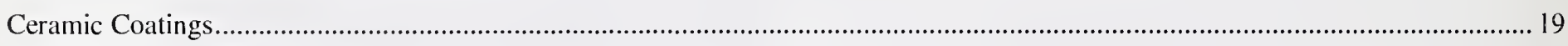

Certification of WC/Co Particle Size Distribution Reference Materials ................................................................................20

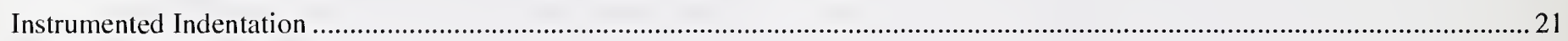

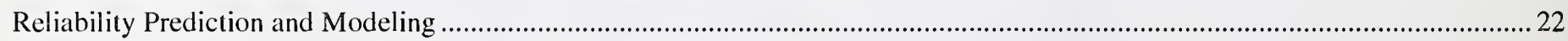

Stress Measurements in Thermally Cycled Thermal Barrier Coatings …...............................................................................2 23

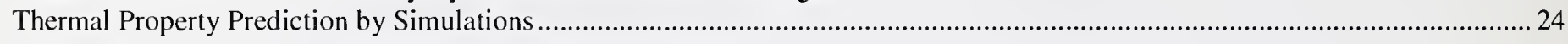

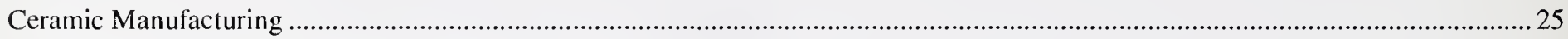

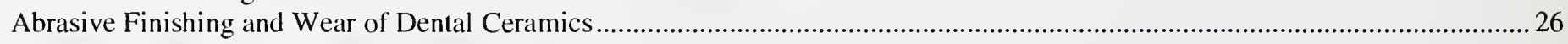

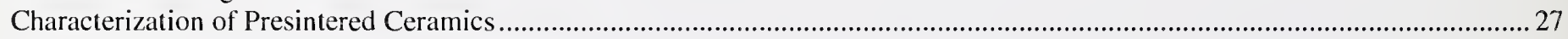

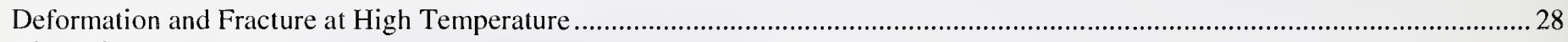

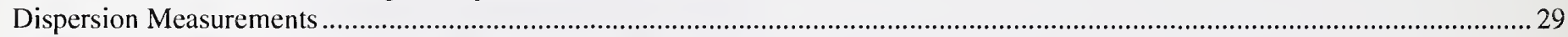

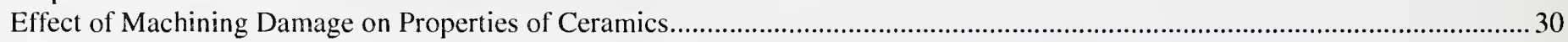

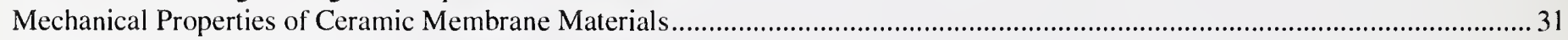

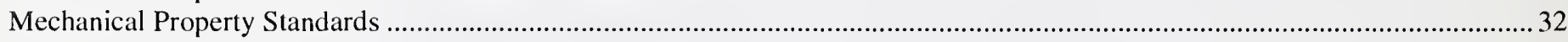

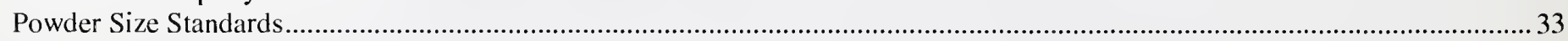

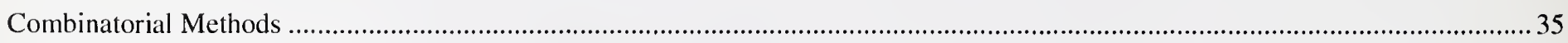

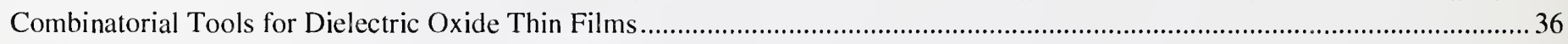

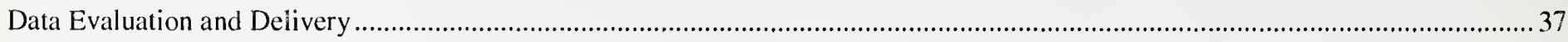

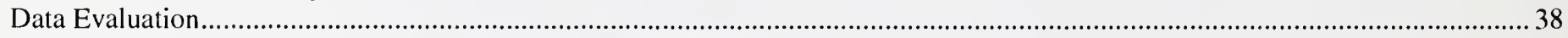

Full Structural Crystallographic Data for Non-Organic Materials...................................................................................... 39

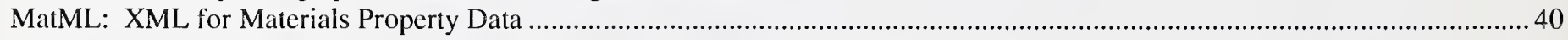

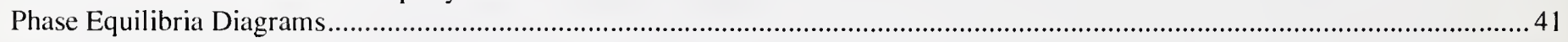

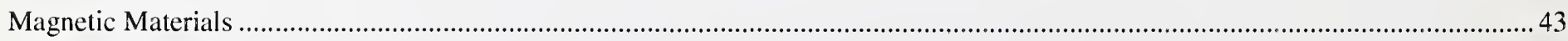

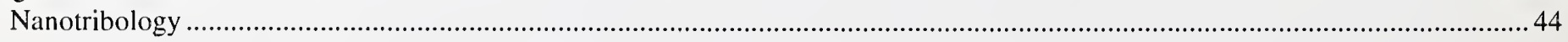

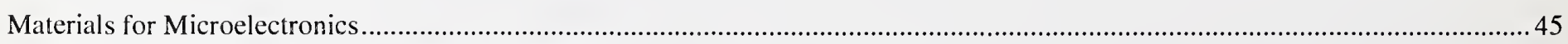

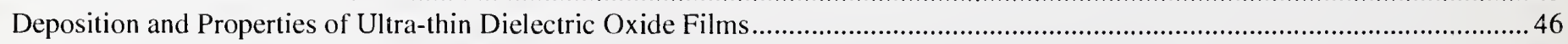

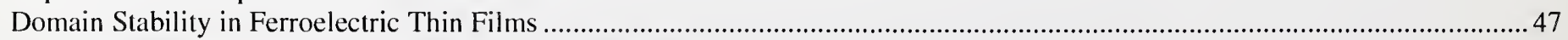

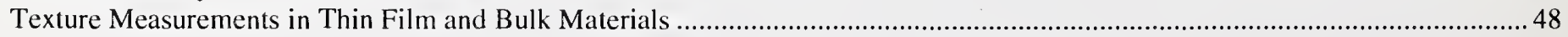


Materials for Wireless Communications.

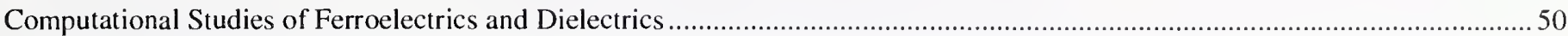

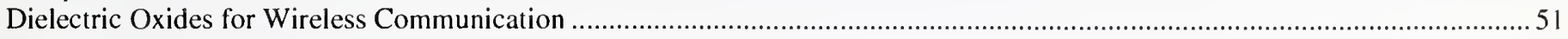

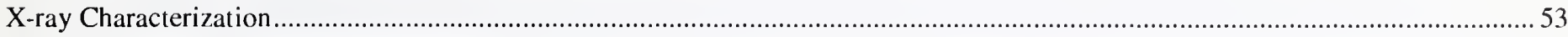

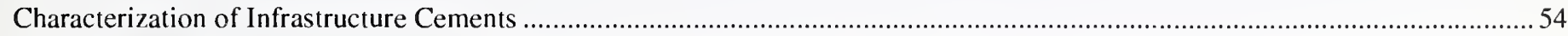

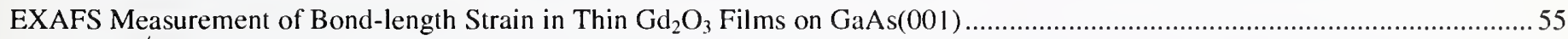

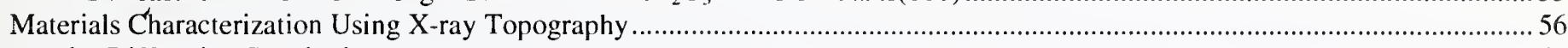

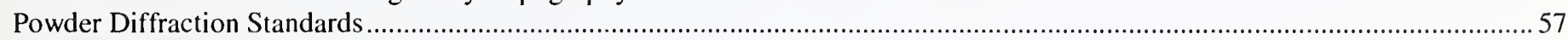

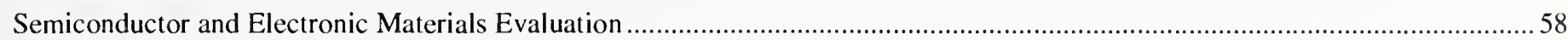

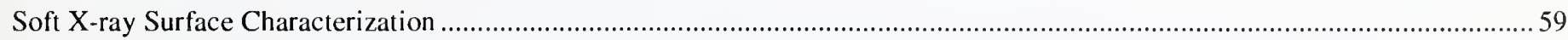

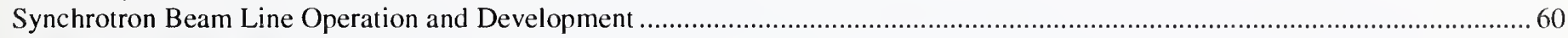

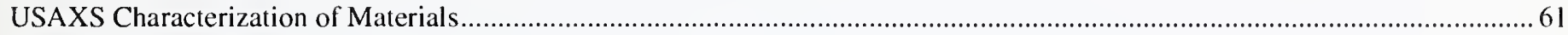

Other

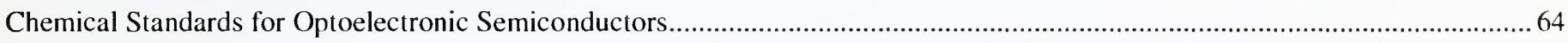

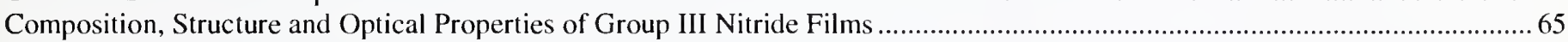

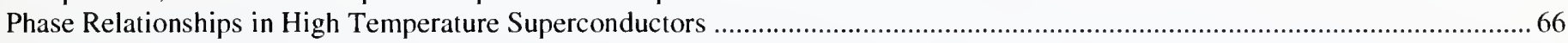

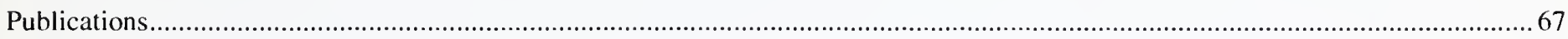

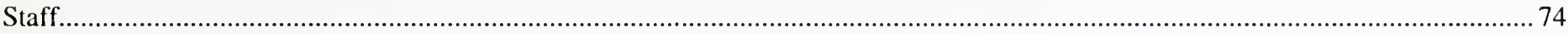

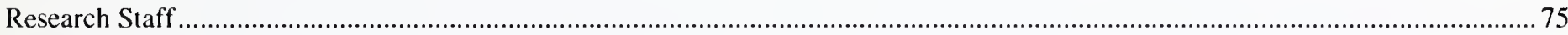

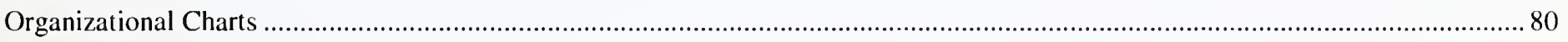

Disclaimer

Certain commercial equipment, instruments, software, or materials are identified in this report to specify adequately experimental procedures, methods of analysis, or material substrates. Such identification does not imply recommendation or endorsement by the National Institute of Standards and Technology, nor does it imply that the equipment, instruments, software, or materials identified are necessarily the best available for the purposes. 



\section{Executive Summary}

The mission of the Ceramics Division is:

Work with industry, standards bodies, academia, and other government agencies in providing the leadership for the Nation's measurements and standards infrastructure for ceramic materials.

The range of ceramic materials on which we work is broad, ranging from oxides and nitrides to materials resembling semiconductors, pertinent to a wide spectrum of important applications. Ceramics in the broadest sense are crucial to many modern technologies including wireless communication, photonics, and biomaterials. Ceramics are used in components, not just as monolithic parts, but as films, coatings, or single crystals. Throughout this range of materials, there are common measurement issues, e.g., phase identification, the role of microstructure on properties, brittle material design, and many others.

We continue to look for improved ways to develop meaningful communication with our customers, i.e. the engineers and scientists in both companies and universities who use the measurement tools that we are developing. In this context, the first NIST Recommended Practice Guide on "Particle Size Characterization", authored by Ajit Jillavenkatesa, Sandy Dapkunas, and Lin Lum, has been completed, and will be published shortly. Practice Guides on "Design with Brittle Materials" and "Nomenclature for Particle Dispersion Technology and Rheological Measurements" will be published later this year.

Workshops continue to be an important way for us to identify industrial measurement needs. During this past year we held workshops related to measurement needs for Low Temperature Co-fired Ceramics (LTCC) for wireless applications, Tribological Issues for Nanotechnology, and Texture Measurements for Ceramic Microstructures.

During the past year, the Ceramic Manufacturing Program, led by Said Jahanmir, was expanded to include topics related to property measurement and reliability prediction of ceramic materials and components, areas of core expertise in the Division for many years. The reorganization was undertaken to better integrate processing with performance prediction.

Based upon the needs expressed at the LTCC Workshop mentioned above, we have also expanded activities related to materials for wireless communication into the area of low temperature co-fired ceramics. In partnership with the NIST Radio-Frequency Technology Division, several follow-on meetings were held with industrial producers and users of LTCC devices. They strongly expressed a need for processing models for these complex, multilayer, devices. This need has led to the initiation of new work within the Division.

Progress was made in our efforts to link the databases in Phase Diagrams and Inorganic Crystal Structures, which we manage with the x-ray diffraction database held by the International Centre for Diffraction Data (ICDD). A Memorandum of Understanding has been agreed to by NIST, the American Ceramic Society, and ICDD. A feasibility study to determine the technical issues involved in this linkage will be initiated shortly.

Our Collaborative Access Team synchrotron beamline at the Advanced Photon Source (APS) in Argonne, Illinois, which we share with the University of Illinois, Oak Ridge National Laboratory, and UOP Corp. was formally dedicated, and is now operational. The APS is the premier synchrotron radiation source in the world, and will provide a unique venue for the development of the next generation of measurements for materials with increasingly smaller features. Our small angle $\mathrm{x}$-ray scattering instrumentation which we developed as part of this partnership is the most frequently used portion of the beamline to date.

Finally, I am extremely pleased that a number of our Division staff have been honored this year. Ed Fuller and Andy Roosen (along with Steve Langer in ITL) received the Jacob Rabinow Applied Research Award for their development of an Object Oriented Finite Element (OOF) analysis; these same individuals, along with Craig Carter at MIT also received a Technology of the Year Award from "Industry Week" for their work on OOF. George Quinn was the recipient of a NIST Bronze Medal. Gabrielle Long received the Maria Goeppert-Mayer distinguished Scholar Award from Argonne National Laboratory. Steve Hsu was made a Fellow of the Society of Tribological and Lubrication Engineers. Winnie Wong-Ng was made a Fellow of ICDD. Doug Blackburn, a retired Division employee, was presented the Sigma Xi Award for outstanding service in support of research scientists. Howard McMurdie, a retired staff member, was given the Barrett Award by the Denver X-ray Conference.

Stephen W. Freiman

Chief, Ceramics Division 



\section{Technical Highlights}

The following Technical Highlights section includes expanded descriptions of research projects that have broad applicability and impact. These projects generally continue for several years. The results are the product of the efforts of several individuals. 


\section{$\mathrm{AO}-\mathrm{Al}_{2} \mathrm{O}_{3}-\mathrm{Nb}_{2} \mathrm{O}_{5}(\mathrm{~A}=\mathrm{Ca}, \mathrm{Sr}, \mathrm{Ba})$ Phase Diagrams Reveal Potentially Useful Wireless Ceramic}

The commercial competitiveness of next-generation base stations depends on improved resonators and filters manufactured from ceramics with better properties and/or reduced processing costs. Experimental phase equilibria and structure-property studies contribute to the fundamental understanding of these ceramics which will eventually permit the rational design of advanced ceramics with controlled dielectric performance. In addition, the existence of potentially useful phases and phase assemblages in selected oxide systems is revealed.

Dielectric oxide ceramics with high permittivity, low dielectric loss, and near-zero temperature dependence of dielectric properties are critical elements in components such as resonators, oscillators, and filters for wireless communications. Relatively few ceramic systems are currently available with the properties needed for practical applications at various operating freqencies. Tantalate-based perovskite derivatives $\mathrm{Ba}_{3} \mathrm{MTa}_{2} \mathrm{O}_{9}$, with $\mathrm{M}=\mathrm{Zn}$ or $\mathrm{Mg}$, are unique in exhibiting permittivities of about 30 together with extremely low dielectric loss tangents $\left(<10^{-5}\right.$ at $\left.1 \mathrm{GHz}\right)$. These studies were motivated by the need for dielectric oxides that could serve as less costly alternatives to the tantalate systems for high-frequency high-power resonator applications. Ternary $\mathrm{Al}_{2} \mathrm{O}_{3}-\mathrm{Nb}_{2} \mathrm{O}_{5}$-containing systems were selected because the polarizability of $\mathrm{Ta}^{5+}$ is intermediate between that of $\mathrm{Al}^{3+}$ and $\mathrm{Nb}^{5+}$, and because similar crystal chemistry is expected.

Subsolidus phase equilibria in the $\mathrm{CaO}: \mathrm{Al}_{2} \mathrm{O}_{3}: \mathrm{Nb}_{2} \mathrm{O}_{5}$ system at $1325^{\circ} \mathrm{C}$ in air were determined. One ternary phase forms, $\mathrm{Ca}_{2} \mathrm{AlNbO}_{6}$, which exhibits a perovskite-related structure with 1:1 or NaCl-type ordering of $\mathrm{Al}^{3+}$ and $\mathrm{Nb}^{5+}$ on the $\mathrm{B}$ sites. Reference $\mathrm{X}$-ray powder diffraction data for this monoclinic compound were prepared. Capacitance methods at $1 \mathrm{MHz}$ were used to determine the dielectric constants and associated temperature coefficients for eleven compounds in this system. The diagram shown at the right includes the resulting relative permittivity values (estimated uncertainty $10 \%$ ) and the signs of their temperature coefficients. As indicated with the red line, $\mathrm{Ca}_{2} \mathrm{AlNbO}_{6}$ and $\mathrm{Ca}_{3} \mathrm{Nb}_{2} \mathrm{O}_{8}$ coexist in equilibria, and were found to exhibit temperature coefficients with opposite signs. Five compositions along this line were prepared and their dielectric properties measured by dielectric resonator methods at 5-7 GHz. A potentially useful temperature-compensated dielectric ceramic was found at the composition

$0.67 \mathrm{Ca}_{2} \mathrm{AlNbO}_{6}: 0.33 \mathrm{Ca}_{3} \mathrm{Nb}_{2} \mathrm{O}_{8}$.

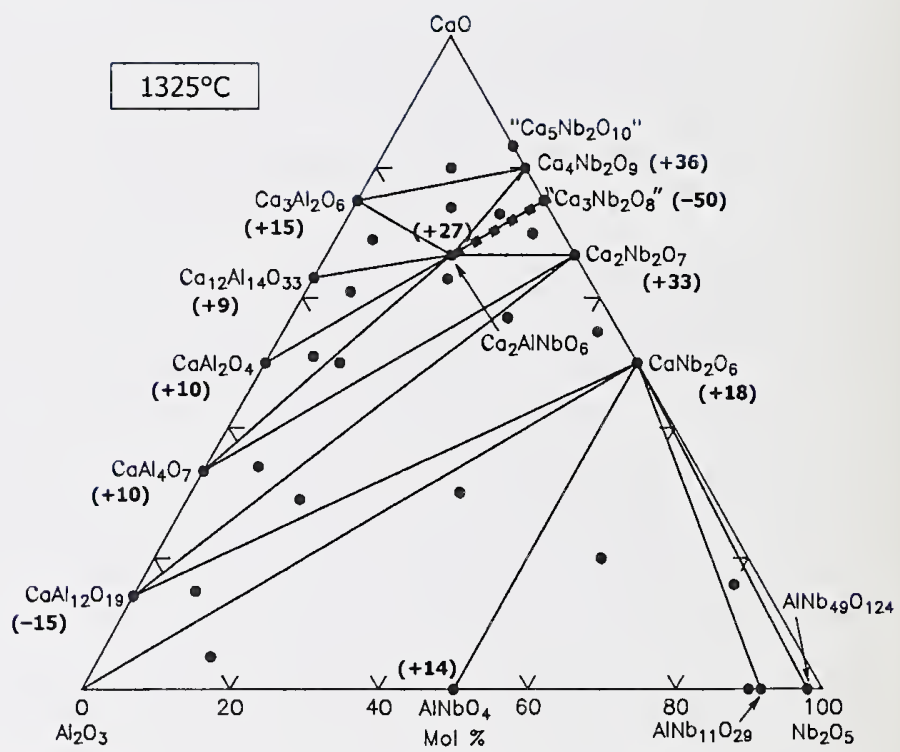

Subsolidus phase equilibria relations found in the $\mathrm{CaO}: \mathrm{Al}_{2} \mathrm{O}_{3}: \mathrm{Nb}_{2} \mathrm{O}_{5}$ system in air. The dielectric constants and the signs of their temperature dependences are included for selected compounds. $\mathrm{Ca}_{2} \mathrm{AlNbO}_{6}$ and $\mathrm{Ca}_{3} \mathrm{Nb}_{2} \mathrm{O}_{8}$ occur in equilibrium with each other and exhibit opposite signs of $\tau_{\varepsilon}$. A stable, temperature-compensated ceramic was found at the composition $0.67 \mathrm{Ca}_{2} \mathrm{AlNbO}_{6}: 0.33 \mathrm{Ca}_{3} \mathrm{Nb}_{2} \mathrm{O}_{8}$.

Subsolidus phase equilibria in the $\mathrm{SrO}-\mathrm{Al}_{2} \mathrm{O}_{3}-\mathrm{Nb}_{2} \mathrm{O}_{5}$ system were determined by synthesis of 75 compositions between $1200{ }^{\circ} \mathrm{C}$ and $1600{ }^{\circ} \mathrm{C}$ in air. As shown in the diagram below, three ternary compounds were observed, including the known double-perovskite, $\mathrm{Sr}_{2} \mathrm{AlNbO}_{6}$, and two new phases, $\mathrm{Sr}_{4} \mathrm{AlNbO}_{8}$ and $\mathrm{Sr}_{5.7} \mathrm{Al}_{0.7} \mathrm{Nb}_{9.3} \mathrm{O}_{30} . \mathrm{Sr}_{4} \mathrm{AlNbO}_{8}$ is unstable above $1525^{\circ} \mathrm{C}$ and forms with a monoclinic unit cell elucidated by electron diffraction and refined using X-ray powder diffraction data; attempts to grow single crystals of this phase for complete structure determination were unsuccessful. $\mathrm{Sr}_{5.7} \mathrm{Al}_{0.7} \mathrm{Nb}_{9.3} \mathrm{O}_{30}\left(\mathrm{Sr}_{6-\mathrm{x}} \mathrm{Al}_{1-\mathrm{x}} \mathrm{Nb}_{9+\mathrm{x}} \mathrm{O}_{30}, \mathrm{x}=0.3\right)$ forms with the tetragonal tungsten bronze structure melts incongruentiy near $1425^{\circ} \mathrm{C}$, and occurs as essentially a point compound, with little or no range of $\mathrm{x}$-values. Difficulties encountered preparing this compound as well as its limited composition region indicate that the tungsten bronze structure 
has a narrow stability region in this chemical system, probably resulting from the relatively small size of $\mathrm{Al}^{3+}$. The existence of an extensive cryolite-like solid solution, $\mathrm{Sr}_{3}\left(\mathrm{Sr}_{1+\mathrm{x}} \mathrm{Nb}_{2-\mathrm{x}}\right) \mathrm{O}_{9}$. $3 / 2 \mathrm{x}$, occurring between $\mathrm{Sr}_{4} \mathrm{Nb}_{2} \mathrm{O}_{9}(\mathrm{x}=0)$ and $\mathrm{Sr}_{6} \mathrm{Nb}_{2} \mathrm{O}_{11}(\mathrm{x}=0.5)$, was confirmed, with cubic lattice parameters. Dielectric properties were measured for the three ternary compounds at frequencies between $3 \mathrm{GHz}$ and $10 \mathrm{GHz}$.

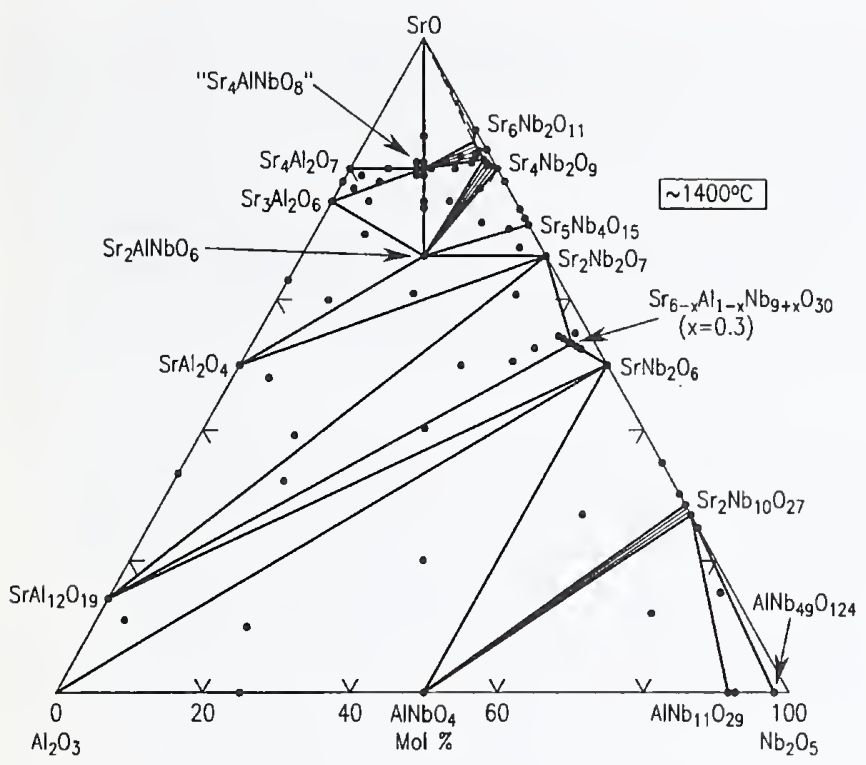

Subsolidus phase equilibria relations in the $\mathrm{SrO}-\mathrm{Al}_{2} \mathrm{O}_{3}-\mathrm{Nb}_{2} \mathrm{O}_{5}$ system determined in air. Three temary compounds were confirmed; " $\mathrm{Sr}_{4} \mathrm{AlNbO}_{8}$ ", $\mathrm{Sr}_{2} \mathrm{AlNbO}_{6}$, and $\mathrm{Sr}_{5.7} \mathrm{Al}_{0.7} \mathrm{Nb}_{9.3} \mathrm{O}_{30}\left(\mathrm{Sr}_{6-\mathrm{x}} \mathrm{Al}_{1-\mathrm{x}} \mathrm{Nb}_{9+\mathrm{x}} \mathrm{O}_{30}, \mathrm{x}=0.3\right)$. The first two compounds exhibit close-packed perovskite-related structures, while the last forms with a framework-type tetragonal tungsten bronze structure.

The subsolidus phase relations in the ternary system $\mathrm{BaO}$ $\mathrm{Al}_{2} \mathrm{O}_{3}-\mathrm{Nb}_{2} \mathrm{O}_{5}$ have also been determined. The phase diagram has revealed the existence of a new ternary composition with the tetragonal tungsten bronze (TTB) structure occurring at the composition $\mathrm{Ba}_{5.75} \mathrm{Al}_{0.75} \mathrm{Nb}_{9.25} \mathrm{O}_{30}\left(\mathrm{Ba}_{6-\mathrm{x}} \mathrm{Al}_{1-\mathrm{x}} \mathrm{Nb}_{9+\mathrm{x}} \mathrm{O}_{30}\right.$, $\mathrm{x}=0.25$ ). As was found in the analogous $\mathrm{SrO}$-system, this TTB-type phase melted incongruently (near $1300^{\circ} \mathrm{C}$ ), and occurs as essentially a point compound, with little or no range of $\mathrm{x}$-values. Variable-temperature capacitance measurements indicated that this compound is nonferroelectric. Solid solutions with TTB-related structures occurring along the $\mathrm{BaO}-\mathrm{Nb}_{2} \mathrm{O}_{5}$ binary were also found to extend into the ternary, dissolving several percent mol fraction $\mathrm{Al}_{2} \mathrm{O}_{3}$.

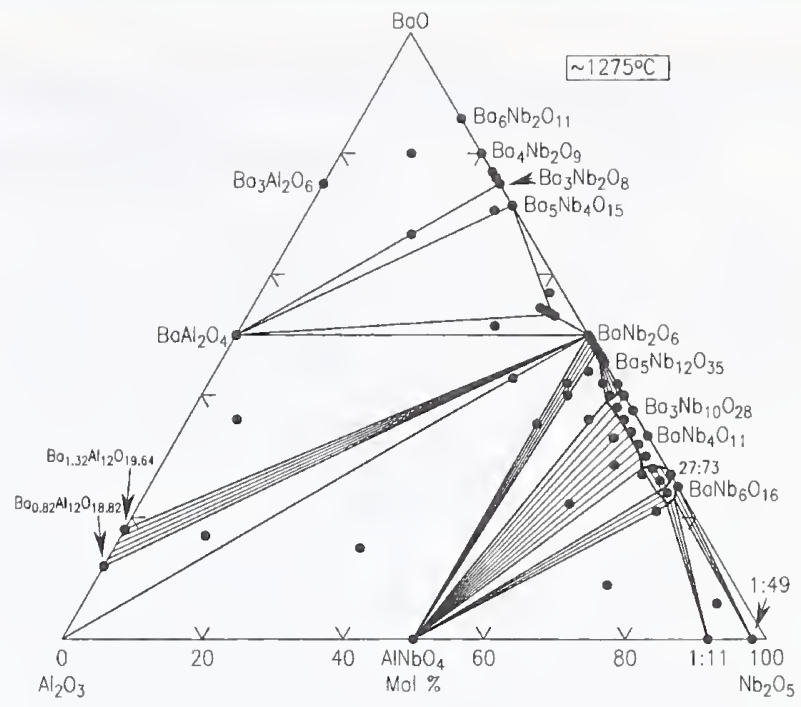

Subsolidus phase relations in the system $\mathrm{BaO}-\mathrm{Al}_{2} \mathrm{O}_{3}-\mathrm{Nb}_{2} \mathrm{O}_{5}$. One ternary compound with the tetragonal tungsten bronze structure was found to occur, along with two single-phase regions with TTB-like structures extending in from the $\mathrm{BaO}-\mathrm{Nb}_{2} \mathrm{O}_{5}$ binary system.

Rolando and Grassi reported phase equilibria in the $\mathrm{MgO}$ $\mathrm{Al}_{2} \mathrm{O}_{3}-\mathrm{Nb}_{2} \mathrm{O}_{5}$ system in 1976 . The study, conducted at $1400^{\circ} \mathrm{C}$, revealed no ternary compound formation, as shown in the diagram here. Considerable solid solution formations were reported; however, the extensive solid solution formation shown here for the phase $\mathrm{AlNbO}_{4}$ was not observed in our studies of the CaO-, SrO-, and $\mathrm{BaO}-$ systems.

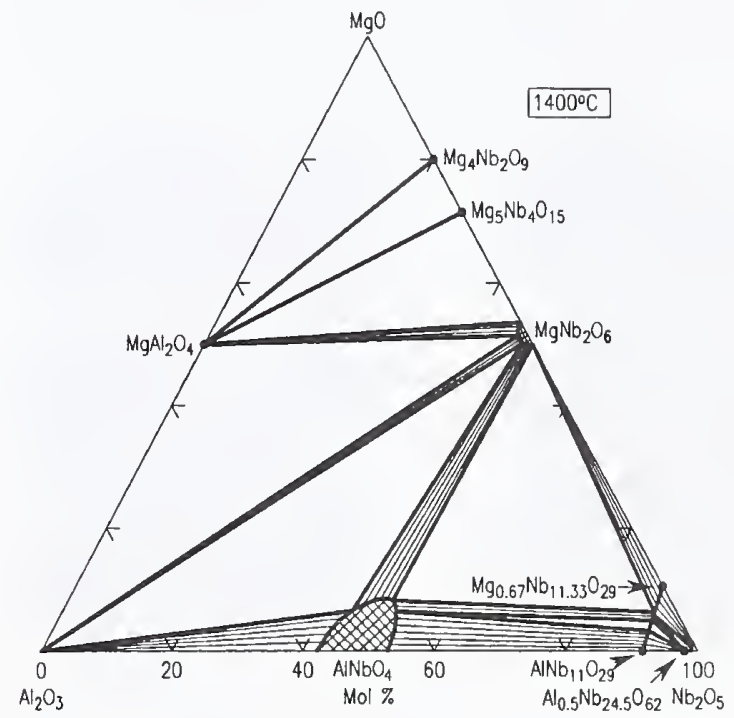

Phase equilibria in the $\mathrm{MgO}-\mathrm{Al}_{2} \mathrm{O}_{3}-\mathrm{Nb}_{2} \mathrm{O}_{5}$ (Rolando \& Grassi, 1976) showing the formation of no ternary compounds, in contrast to the other systerns
For More Information On This Topic
T.A. Vanderah, W. Febo, Julia Y. Chan, R.S. Roth, J.M. Loezos, L.D. Rotter, R.G. Geyer, and D.B. Minor, "Phase Equilibria and Dielectric Behavior in the $\mathrm{CaO}-\mathrm{Al}_{2} \mathrm{O}_{3}$ $\mathrm{Nb}_{2} \mathrm{O}_{5}$ System", J. Solid State Chem. 154, xxx (2000).

Julia Y. Chan, I. Levin, T.A. Vanderah, R.G. Geyer, and R.S. Roth, "Subsolidus Phase Relations and Dielectric Properties in the $\mathrm{SrO}-\mathrm{Al}_{2} \mathrm{O}_{3}-\mathrm{Nb}_{2} \mathrm{O}_{5}$ System", Int' $t$. J. Inorg. Mats. 2(1), 107-114 (2000).

Contact: T.A. Vanderah, I. Levin 


\section{First-Principles Calculation of Dielectric Permittivity}

\section{in Microwave Materials}

One of the criteria of dielectric materials for microwave applications is that they have high permittivity (dielectric constant). In this project, we demonstrated that density-functional theory methods, using only the crystallographic cell parameters and space group as experimental input, can be used to compute dielectric permittivities, and that good agreement with experiment is obtained. In particular, we focused on $\mathrm{CaTiO}_{3}$ and $\mathrm{CaAl}_{1 / 2} \mathrm{Nb}_{1 / 2} \mathrm{O}_{3}$. These two materials have nearly the same structure, yet the pernittivity of $\mathrm{CaTiO}_{3}$ is nearly 10 times larger than that of $\mathrm{CaAl}_{1 / 2} \mathrm{Nb}_{1 / 2} \mathrm{O}_{3}$. Our calculations reproduce the different dielectric behaviors.

Furthermore, they show that the differences in permittivity are due in large part to the different phonon properties of the lowestfrequency phonons in the two systems and suggest that these differences in turn are related to differences in electronic structure.

Dielectric materials for microwave applications must have high permittivity, low loss, and temperature stability. Uncovering the relationship between chemistry, structure, and dielectric properties is of the utmost importance for the rational design of new dielectric materials. The contrasting dielectric properties of the closely related materials $\mathrm{CaTiO}_{3}$ (CT) and $\mathrm{CaAl}_{1 / 2} \mathrm{Nb}_{1 / 2} \mathrm{O}_{3}$ (CAN) highlight the need for fundamental studies of structure-property relationships. CT is a simple perovskite; CAN is a doubled perovskite with a rocksalt-like $1: 1$ ordering of $\mathrm{Al}$ and $\mathrm{Nb}$ on the $\mathrm{B}$-site sublattice. CT and CAN have nearly identical lattice parameters, tolerance factors, and octahedral tilting patterns and magnitudes (Figure 1).
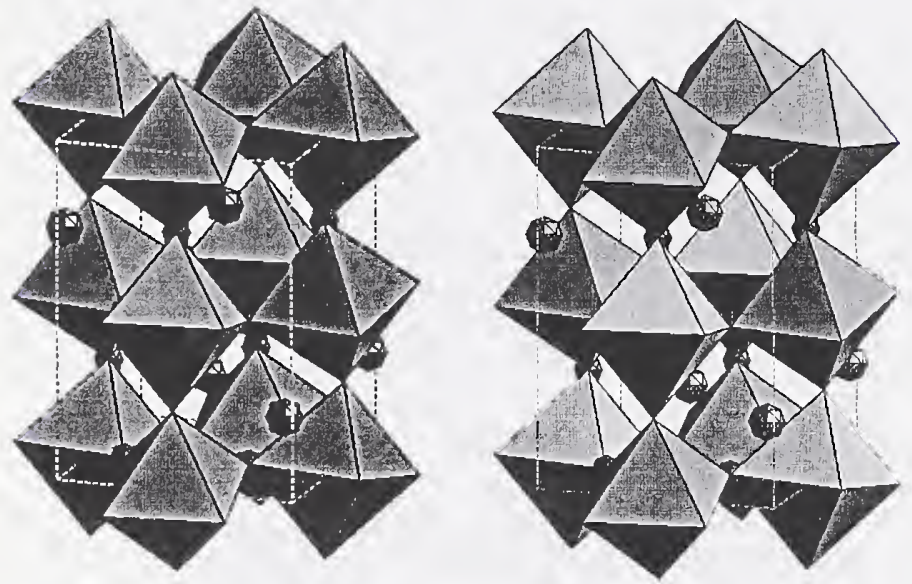

Figure 1

Experimentally, however, CT has dielectric permittivity $\kappa \sim$ 170 and and a temperature coefficient of resonance frequency $\tau_{\mathrm{f}} \sim+900$ at room temperature whereas CAN has $\kappa \sim 27$ and $\tau_{\mathrm{f}} \sim-88 \mathrm{MK}^{-1}$. Furthermore, the CT-CAN system is of experimental interest because it forms a solid solution series across the entire composition range and thus contains a single-phase material with $\tau_{\mathrm{f}}=0$, which is potentially a useful microwave dielectric material.

All calculations are performed using VASP (the Vienna ab initio simulation package). VASP is a code for plane-wave pseudopotential density functional theory calculations. The highest-precision options were used for well-converged results. We used the experimental lattice parameters and space group as the only input. The ionic positions in each system were relaxed to equilibrium. Then, by perturbing each ionic positions in the relaxed structure in turn and calculating the change in interatomic forces and polarization, we obtained the phonon properties of each system.

In order to understand the structure-property relationships that lead the simple perovskite CT to have a higher permittivity than the the double perovskite CAN, we turned to first-principles calculations. All calculations are performed using VASP (the Vienna ab initio simulation package) which is a code for plane-wave pseudopotential density functional theory calculations. We used the experimental lattice parameters and space group as the only input. The ionic positions in each system were relaxed to equilibrium. Then, by perturbing each ionic positions in the relaxed structure in turn and calculating the change in interatomic forces and polarization, we obtained the phonon properties of each system.

Finally, dispersion theory is used to relate the dielectric constant of a system to its calculated phonon properties. The results are shown in Figure 2. The computed dielectric constant is 275.0 for CT and 25.3 for CAN. These compare very well with the experimental values. The reasons for the discrepancies are two-fold. First, whereas density-functional theory is exact in principle, the exact formula is unknown. Any result obtained is inexact. Second, the dispersion formula assumes a harmonic system. In real systems, interatomic forces are anharmonic which affects the dielectric properties.

Nonetheless, the agreement with experiment is good enough to allow us to understand the reasons for the different dielectric behaviors. As can be seen in Figure 2, the dielectric response of CT is dominated by that of its three lowest-frequency phonons near $100 \mathrm{~cm}^{-1}$. The corresponding phonons in CAN occur at higher frequencies and with lower effective charges than in CT. Both trends suppress the dielectric constant, which mathematically depends on the ratio of the effective charge to frequency. 

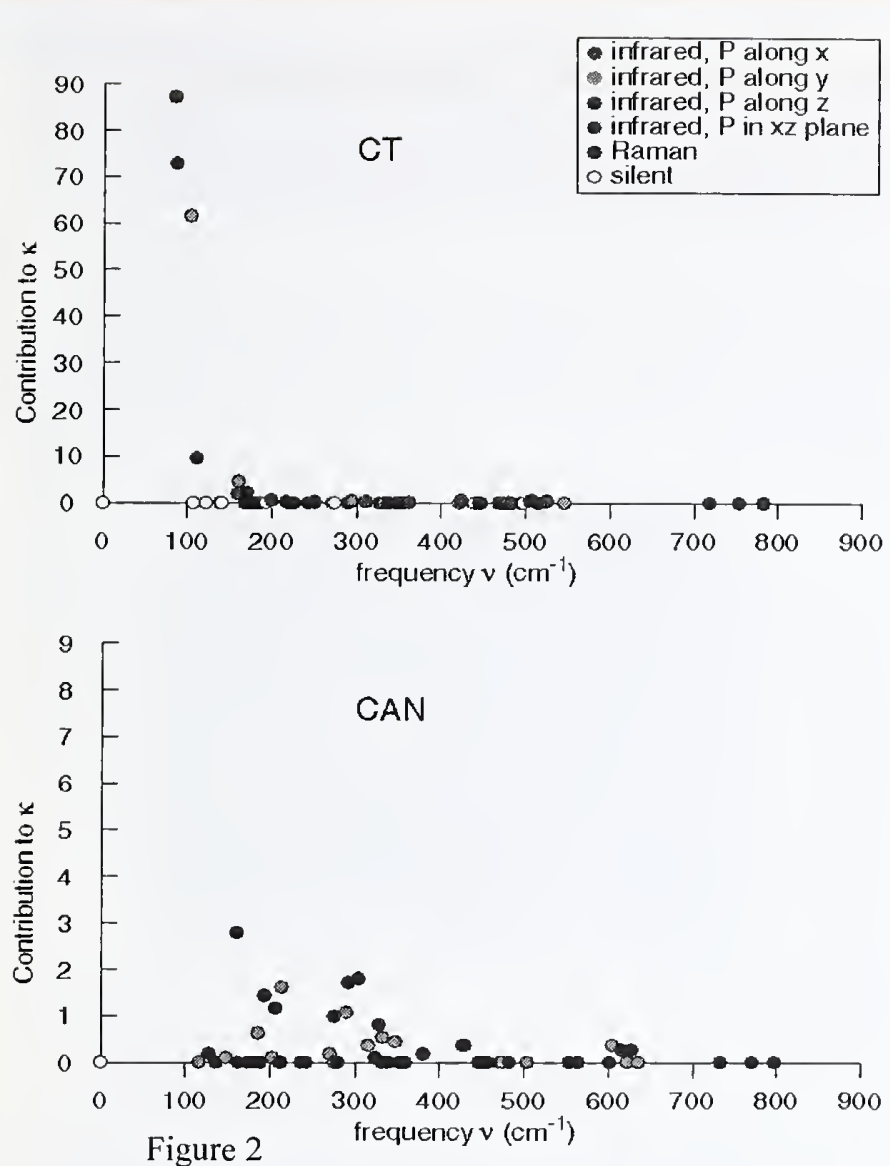

The differences in frequencies arise from all the interatomic forces and it is difficult to pinpoint the reasons for the differences. The different effective charges on the other hand, are clearly related to differences in the nature of the phonons eigenvectors (Figure 3 ). In $\mathrm{CT}$, both $\mathrm{Ca}$ and $\mathrm{Ti}$ move against the oxygens in the lowest frequency polar phonons. The net polarization is maximized when positive and negative charges move in opposite directions. In CAN, on the other hand, $\mathrm{Ca}$ moves against the $\mathrm{O}$, but $\mathrm{Al}$ and $\mathrm{Nb}$ both move with the oxygens, which suppresses the overall charge displacement.
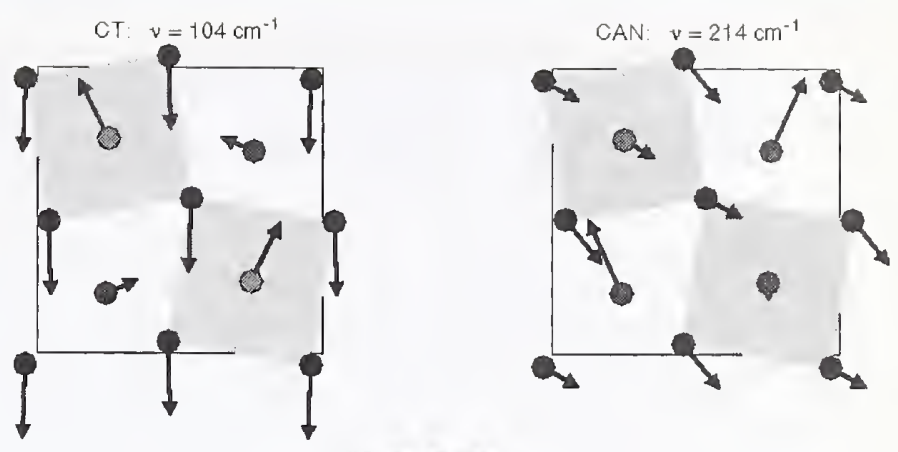

Figure 3

Why do B site cations ( $\mathrm{Ti}, \mathrm{Al}, \mathrm{Nb}$ ) move against the $\mathrm{O}$ in $\mathrm{CT}$ but not in CAN? We first note that in the case of simple perovskites involving $\mathrm{Nb}$, such as $\mathrm{KNbO}_{3}(\mathrm{KN}), \mathrm{Nb}$ moves against the oxygens in the lowest-frequency polar modes. This difference in the behavior of $\mathrm{Nb}$ in CAN and $\mathrm{KN}$ is not due to size effects: the $\mathrm{Nb}-\mathrm{O}$ distances in the two compounds are almost identical. We look instead at differences in electronic structure.

It is well known that hybridization of oxygen $2 p$ and $B$ site transition metal $\mathrm{d}$ electrons is very important for the lattice dynamics of perovskites. One expects an inverse relationship between the importance of covalent bonding in a perovskite and the band gap, all other factors being the same. With this in mind, we calculated the band gaps in CT, CAN, and KN. Our results support the hypothesis that larger band gaps lead to reduced importance of covalent bonding and less tendency of B site cations to move against the oxygens in the lowfrequency phonons. The reason for the larger gap in CAN than $\mathrm{KN}$ is due to the presence of the $\mathrm{Al}$. The $\mathrm{Al}$ ions contribute no low-lying energy levels to the conduction bands. The lowest-lying conduction states come mainly from $\mathrm{Nb}-4 \mathrm{~d}$ electrons. In a simple perovskite with $\mathrm{Nb}$ on every $\mathrm{B}$ site, the lowest conduction state wavefunction is spread over all the B sites and is more plane-wave-like than in a complex perovskite where $\mathrm{Nb}$ and thus the wavefunction is distributed on only half the sites. The more localized wavefunctions in the complex perovskite lead to higher eigenenergies and thus a larger band gap. It will be interesting to see how the type and ordering of the $\mathrm{B}$ site cations affect the electronic structure, phonon behavior, and dielectric properties of complex perovskites in general.
For More Information On This Topic
Cockayne, E., "Comparative Dielectric Response in $\mathrm{CaTiO}_{3}$ and $\mathrm{CaAl}_{1 / 2} \mathrm{Nb}_{1 / 2} \mathrm{O}_{3}$ from First Principles", submitted to J. Appl. Phys.

Contact person: Eric Cockayne. 


\section{New Methodologies to Characterize Machining Damage and Its Effect on Strength in Ceramics}

Cost effective means to machine ceramics with a minimum of machining induced surface damage are of considerable importance. One component of the NIST Ceramics Division's Ceramic Machining Consortium and Mechanical Test Method Standardization programs has been a study to characterize and evaluate the effects of machining on ground surfaces of ceramic cylindrical rods and rectangular test bars. Test methods to evaluate machining damage and fractographic tools to detect machining cracks have been developed and refined.

Manufacturing processes for a variety of ceramic materials and applications require grinding for shaping and final finishing. When applied to load bearing ceramic components, a critical aspect of a grinding process is the extent to which grinding induced damage affects the strength and reliability of the component. Thus, measurements to determine the effects of grinding damage and the characterization of grinding damage are critical to the development of cost effective ceramic grinding processes. Three significant accomplishments for this year have been (1) development of simple fractographic means for identification of machining cracks, (2) finalization of the development of a test method for measuring the flexure strength of cylindrical rod specimens), and (3) completion of a comprehensive analysis on a particular sintered reactionbonded silicon nitride. Much of the work leading to these accomplishments was conducted in association with projects undertaken by the NIST Ceramic Machining Consortium. An important goal of the Consortium has been the determination of the effect of different grinding conditions on the flexure strength of commercially significant ceramic materials. Realization of this goal has required the development of new test methods and better means for the identification machining damage.

Machining cracks can be difficult to detect on fracture surfaces even if a fracture mirror identifies the fracture origin area. Machining cracks that extend beneath the surface by $20 \mu_{\mathrm{m}}$ to $50 \mu_{\mathrm{m}}$ often blend into the background microstructure, especially in ceramics with elongated grain microstructures designed to enhance toughness. The microstructural irregularities create a rougher fracture surface and subtle machining cracks may be impossible or difficult to detect. In the past, the usual practice has been to grind then break some bend bars. If a strength loss relative to some baseline value was detected, it was inferred that machining cracks must have been present. Sometimes this analysis was supplemented by rudimentary fractographic examination to at least verify that the origins were located on the specimen surface. This approach was not infallible, however, since many inherent material flaws may reside by chance at or near the surface. It is unreasonable to arbitrarily characterize such origins as machining flaws.
A new simple fractographic means to detect transverse machining cracks with a minimum of fuss has been discovered. Some cracks have characteristic telltale features that can be detected with low power optical microscopy. In other cases, the fracture mirror itself has certain telltale, noncircular shapes and it could reliably be inferred that machining damage or a scratch was strength limiting without actually seeing the machining crack itself! The new practices are general and not material specific and they may be used by even novice fractographers. The telltale features have been detected in other ceramics, glasses, and even single crystals. These new fractographic methods will be incorporated into several ASTM standards.
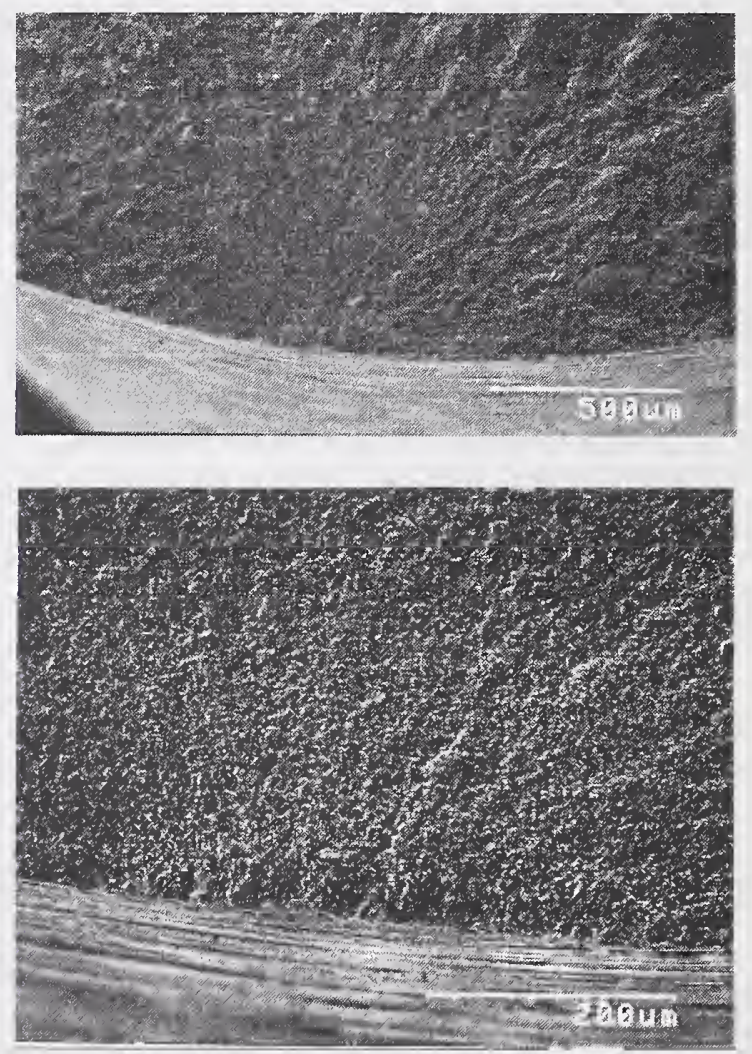

Figure 1 Fracture surface of a ground silicon nitride rod with a machining crack fracture origin. 
Flexure testing may be used to investigation the effects of surface grinding processes on strength. In the past, strength measurements have predominantly been on rectangular bars using standard test methods. However, only planar grinding modes are used to machine rectangular bars, effectively limiting such investigations to those modes of grinding. In fact, the majority of ceramic parts are cylindrical or circular in cross section requiring cylindrical and centerless grinding modes for fabrication. In response to this clearly identified need, a project was undertaken to develop flexure test methods applicable to cylindrical rods. This effort has led to the design of a flexure test fixture (shown below) and verification of its performance.

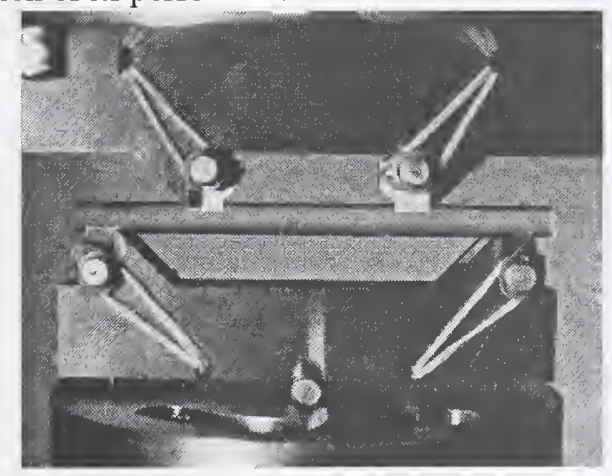

Figure 2 The new fixture for flexural strength testing with a glass rod specimen.

The cylindrical rod flexure test method was applied to a Consortium project designed to investigate the effect of finish grinding conditions on the flexure strength of a commercial sintered reaction-bonded silicon nitride that is in use in heavy-duty diesel engines. The engine component is cylindrical in shape and finish machining by centerless grinding is a key step in the manufacturing process.

Figure 3 shows the results of the study. The figure is a Weibull probability plot showing the distribution of flexure strengths of sets of rods and rectangular bars. The rods were centerless or cylindrically ground. Baseline strength data were obtained by grinding in the longitudinal direction with a 320 grit resin bond diamond wheel. Using the same wheel and grinding conditions, but grinding in the transverse direction, it is seen that flexure strength is substantially lower. When the same experiment was repeated with a finer 600 grit resin bond wheel, the flexure strengths in the longitudinal and transverse directions are nearly the same. Moreover, these strengths differ little from values obtained for the 320 grit wheel in the longitudinal direction.

Accordingly, it might be hypothesized that, for these sets of specimens, fracture did not originate at grinding damage and probably initiated at intrinsic flaws in the material.
A detailed fractographic examination was conducted on all specimens and it confirmed that fracture originated at inclusions, pores, sintering irregularities, or other microstructural imperfections inherent to the material. In contrast, by utilizing the fractography methods described earlier it was found that fracture of the 320 grit transverse ground specimens was due to machining damage. A key finding from this work was that 600 grit transverse finish grinding created negligible or minor damage to this particular silicon nitride. Such machining is simpler and more cost effective than longitudinal grinding in manufacturing cylindrical parts.

The new flexure test methodology for cylindrical rods has been refined to the point that testing is routine and just as easy as testing the more traditional rectangular bend bars. It is anticipated that this will open the way to flexural strength investigations with the associated centerless and cylindrical modes of grinding. A new ASTM standard for cylindrical rod flexure testing will be the focus of work in FY 2001.

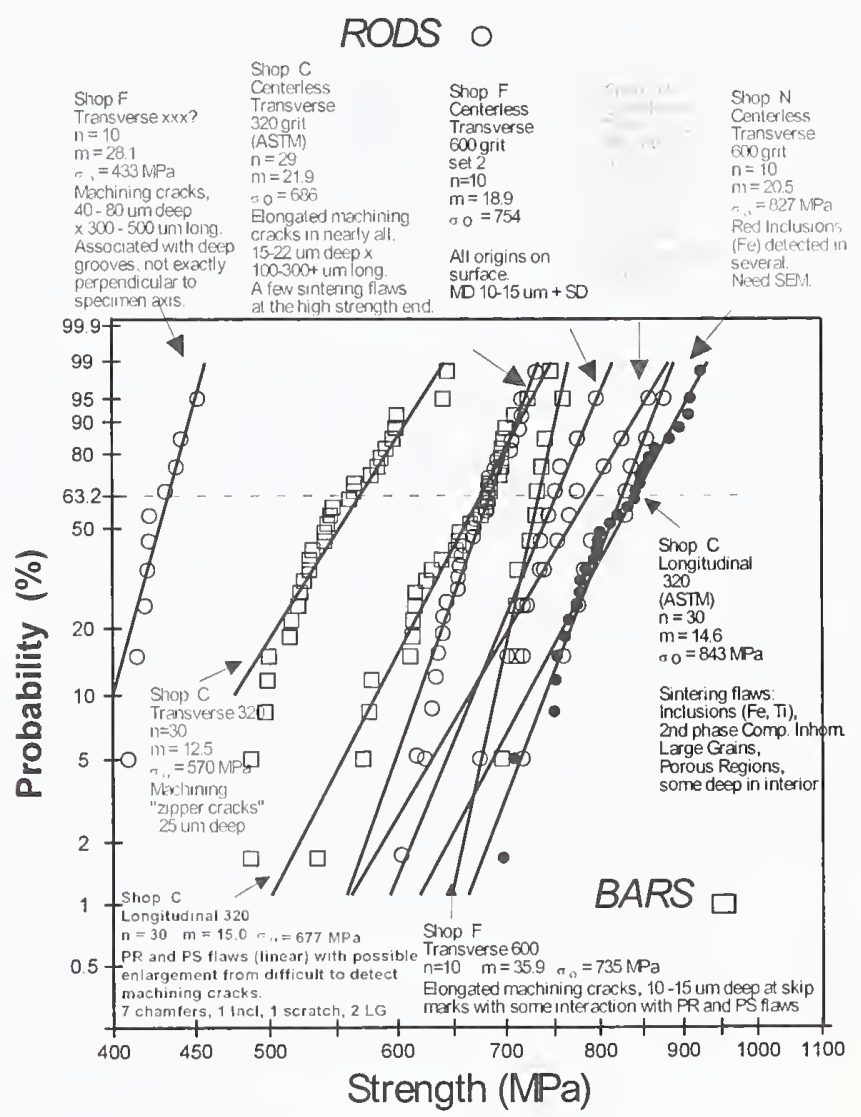

Figure 3 Weibull distribution graph of flexural strengths for a commercial silicon nitride.
For More Information

On This Topic
Publications: G. D. Quinn, L. K. Ives, S. Jahanmir, and P. Koshy, "Fractographic Analysis of Machining Cracks in Silicon Nitride Rods and Bars," presented at the 4th International Conference on the Fractography of Glasses and Ceramics, Alfred University, July 2000, to be publ., Ceramic Transactions, eds, J. Varner, G. Quinn, V. Frechette, American Ceramic Society, Westerville, OH, 2001. 


\section{NIST Recommended Practice Guide}

In 2000, we produced the first NIST Recommended Practice Guide, SP 960-1, “ Particle Size Characterization" by Ajit Jillavenkatesa, Stanley Dapkunas, and Lin-Sien Lum. The Guide synthesizes information on the measurement of ceramic particle size, providing a broad perspective of methods of size measurement, sampling, data analysis, and identification of relevant standards

The Recommended Practice Guide series is a new approach for delivering information to the ceramics community and provides an easily accessed and understandable source of practical knowledge. The Practice Guide series synthesizes the wealth of experience and data developed at NIST over many years on specific topics. This information is generally published in the scientific literature over several years and may not be readily available. The Guide series is intended for scientists and engineers who are not familiar with particular measurement technologies and who require a general perspective.

Determination of particle size distribution is a critical step in almost all ceramic processing techniques. However, particle size analysis techniques are often applied and/or results interpreted inappropriately, primarily due to a lack of understanding of the underlying principles of size analysis, or due to confusion arising from claims and counter-claims of the analytical ability of size determination techniques and instruments. "Particle Size Characterization" has been written to address some of these issues and concerns. The Guide is not an exhaustive and comprehensive text on particle size analysis, but attempts to convey the practical issues that need to be considered when conducting analysis by some of the techniques commonly used in the ceramics community. Data and information from studies conducted over years of participation in international round robin tests and standards development have been used in developing the information in the Guide. The Guide also provides comparative data on different measurement methods applied to the same material, as illustrated in Figure 1.

"Particle Size Characterization" explains the physical principals involved for most broadly used methods of measurement or characterization, provides general procedures for each method, identifies sources of error and variation, describes methods of data analysis, and lists relevant national and international standards. The Guide was reviewed by individuals in industry to ensure that it provides information that is both useful and usable by those concerned with particle size characterization.
SRM 1982 Particle Size Distribution

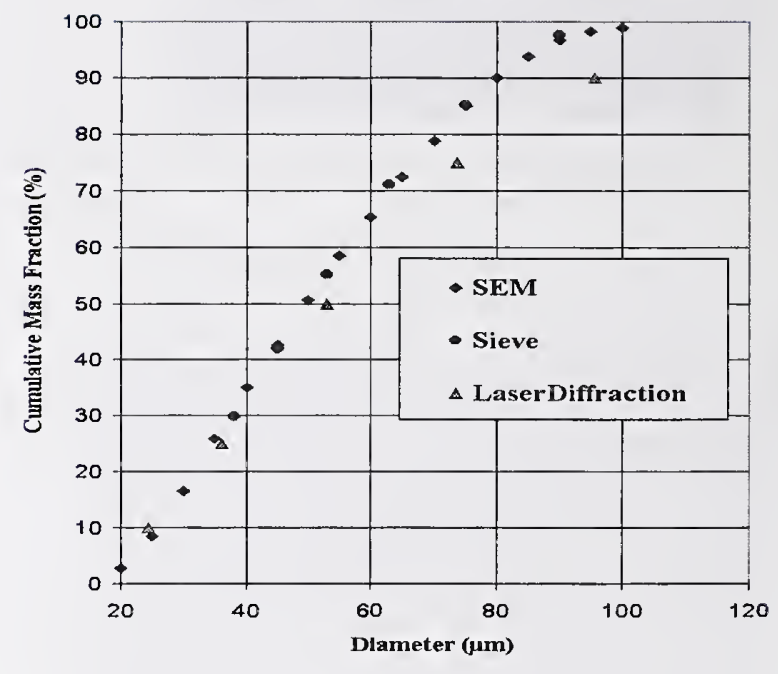

Figure 1. Comparison of Particle Size Distribution for NIST SRM 1982 Measured by Three Techniques

Specific topics covered in "Particle Size Characterization" include:

- powder sampling,

- sieving techniques,

- x-ray gravitational sedimentation techniques,

- microscopy based techniques,

- laser light diffraction techniques,

- reporting size data; and

- a glossary of terms.

The NIST Recommended Practice Guide for Particle Size Characterization, Figure 2, will be followed by two other Guides for the ceramics community: Nomenclature for Particle Dispersion Technology and Rheological Measurements; and, Design with Brittle Materials. 


\section{practice guide}

\section{Particle Size Characterization}

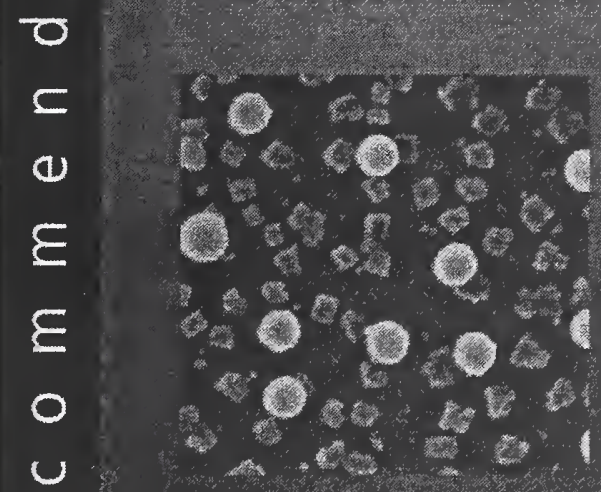

(1) Ajit Jillavenkatesa

Stanley 1. Dapkunas

Lin-Sien H. Lum

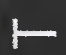

U SP 960-1

- NGT

2

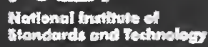

Fodroiogr Adminitwolion

Figure 2. NIST Recommended Practice Guide, SP 960-1, "Particle Size Characterization" (picture of the cover)

The NIST Recommended Practice Guide for Particle Size

Characterization may be obtained from the Ceramics Division office at 301/975-6119 or carolyn.sladic@nist.gov. The Guide will also be available at the Ceramics Division Website, www.ceramics.nist.gov.

\section{For More Information}

On This Topic

Ajit Jillavenkatesa and S. J. Dapkunas, Ceramics Division 


\section{Phase Equilibria of Ba-Nd-Cu-O, and Critical Single- Phase Region for Tailoring Superconducting Properties of $\mathrm{Ba}_{2-x}\left(\mathrm{Nd}_{1+x-y} \mathrm{Yb}_{\mathrm{y}}\right) \mathrm{Cu}_{3} \mathrm{O}_{6+z}$}

By mixing of the smaller $\mathrm{Yb}$ ion with $\mathrm{Nd}$ in $\mathrm{Ba}_{2-x} \mathrm{Nd}_{1+x} \mathrm{Cu}_{3} \mathrm{O}_{6+z}(\mathrm{Nd}-213)$ superconductor, both flux-pinning and melting properties can be tailored and optimized. To provide the basis for this effort, we have determined the phase diagrant of the $\mathrm{BaO}_{-} \mathrm{Nd}_{2} \mathrm{O}_{3}-\mathrm{CuO} \mathrm{O}_{x}$ system, as well as the single plase region of $\mathrm{Ba}_{2-x}\left(\mathrm{Nd}_{1+x-y} \mathrm{Yb}_{y}\right) \mathrm{Cu}_{3} \mathrm{O}_{6+z}$ under atmospherically controlled conditions. The diagrams of $\mathrm{BaO}-\mathrm{Nd}_{2} \mathrm{O}_{3}-\mathrm{Cu}_{2} \mathrm{O}_{x}$ determined under purified air and $0.1 \% \mathrm{O}_{2}$ are similar, but substantially different from the literature data, which were determined using $\mathrm{BaCO}_{3}$. The extent of the solid solution decreases, while tle onset temperature of the $T_{c}$ values increase as the oxygen partial pressure decreases. The locations of the single-phase boundaries provide information important for processing flux-

pinned materials, and will facilitate rapid advancement of the second generation RABiTS/IBAD coated conductor technology.

The relatively recent success in preparing $\mathrm{BaR}_{2} \mathrm{Cu}_{3} \mathrm{O}_{6+2}$ materials with promising superconducting properties via the RABiTS and IBAD processes has revitalized research interest in $\mathrm{BaO}-\mathrm{R}_{2} \mathrm{O}_{3}-\mathrm{CuO}_{\mathrm{x}}$ systems. In particular, the $\mathrm{BaO}$ $\mathrm{Nd}_{2} \mathrm{O}_{3}-\mathrm{CuO}_{\mathrm{x}}$ system is one of the most promising lanthanide systems for the development of high $T_{c}$ superconductors. $\mathrm{BaNd}_{2} \mathrm{Cu}_{3} \mathrm{O}_{6+Z}(\mathrm{Nd}-213)$ demonstrates a high $\mathrm{T}_{\mathrm{c}}$ value as well as high vortex-pinning by comparison with

$\mathrm{Ba}_{2} \mathrm{YCu}_{3} \mathrm{O}_{6+\times}$. Because of the formation of a large region of solid solution in $\mathrm{Ba}_{2-x} \mathrm{Nd}_{1+\mathrm{x}} \mathrm{Cu}_{3} \mathrm{O}_{2}$ associated with the substitution of $\mathrm{Nd}$ on the $\mathrm{Ba}$ site, one can control physical properties by varying the solid solution composition. Furthermore, by substituting $\mathrm{Nd}$ with the smaller $\mathrm{Yb}$ ion, one can tailor both superconducting and melting properties of $\mathrm{Ba}_{2-\mathrm{x}}\left(\mathrm{Nd}_{1+x-\mathrm{y}} \mathrm{Yb}_{\mathrm{y}}\right) \mathrm{Cu}_{3} \mathrm{O}_{6+z}$.

An experimental procedure has been successfully established to study phase diagrams under controlled atmosphere while minimizing the presence of $\mathrm{CO}_{2}$, which results in the formation of carbonates, especially in the presence of $\mathrm{BaO}$, and incorrect phase equilibria data.

The diagrams of $\mathrm{BaO}-\mathrm{Nd}_{2} \mathrm{O}_{3}-\mathrm{CuO}_{\mathrm{x}}$ determined under purified air and $0.1 \% \mathrm{O}_{2}$ (Fig. l) are very similar, but substantially different from the literature data, which were determined using $\mathrm{BaCO}_{3}$. The $\mathrm{BaO}-\mathrm{Nd}_{2} \mathrm{O}_{3}-\mathrm{CuO}_{\mathrm{x}}$ system consists of three solid solutions and one stoichiometric compound. The first solid solution is the high $\mathrm{T}_{\mathrm{c}}$ series, $\mathrm{Ba}_{2-\mathrm{x}} \mathrm{Nd}_{1+\mathrm{x}} \mathrm{Cu}_{3} \mathrm{O}_{2}$. We have confirmed that the extent of this solid solution is greater than previously reported, and that it extends from $x=0$ to 0.95 . Two phase transformations occur within this solid solution instead of the commonly known single transformation. These transformations progress from orthorhombic tetragonal and then to orthorhombic (Fig. 2), according to electron microscopy studies. The second series, $\mathrm{Ba}_{1+x} \mathrm{Nd}_{2-x} \mathrm{CuO}_{2}$, (the 'brown-phase') has only a narrow solid solution region when prepared in air or in $0.1 \% \mathrm{O}_{2}$. $\mathrm{Ba}_{2} \mathrm{Nd}_{4} \mathrm{Cu}_{2} \mathrm{O}_{2}$ was found to be antiferromagnetic, but showed ferromagnetic behavior as $\mathrm{Nd}$ was partially substituted by $\mathrm{La}$ The $\mathrm{Ba}_{2}(\mathrm{Nd}, \mathrm{La})_{4} \mathrm{Cu}_{2} \mathrm{O}_{2}$ phase may provide desirable magnetic pinning centers for processed $\mathrm{Nd}-213$ ceramics. In the high Ba-content region, the orthorhombic solid solution

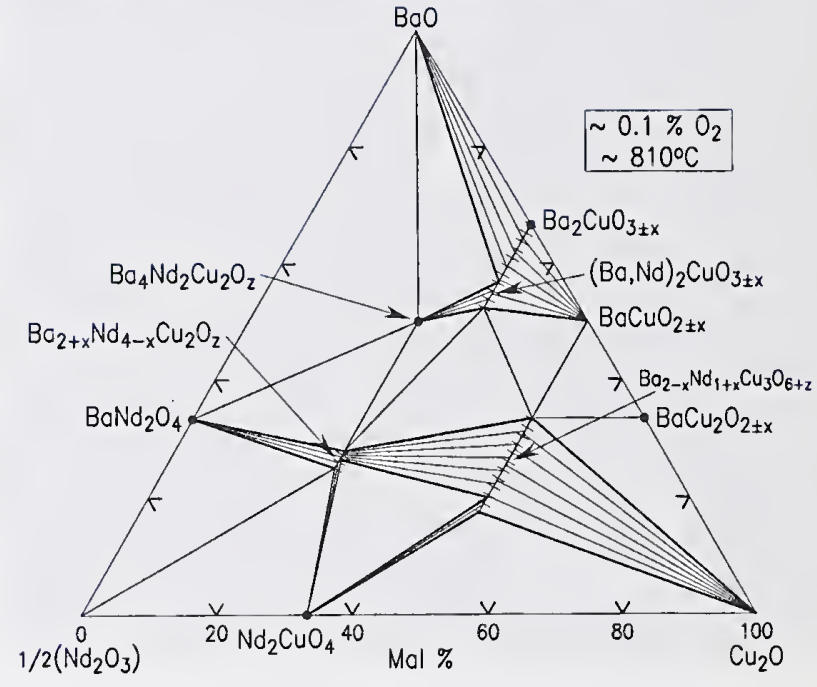

Fig. 1. Phase diagram of the $\mathrm{BaO}-\mathrm{Nd}_{2} \mathrm{O}_{3}-\mathrm{CuO}_{\mathrm{x}}$ system

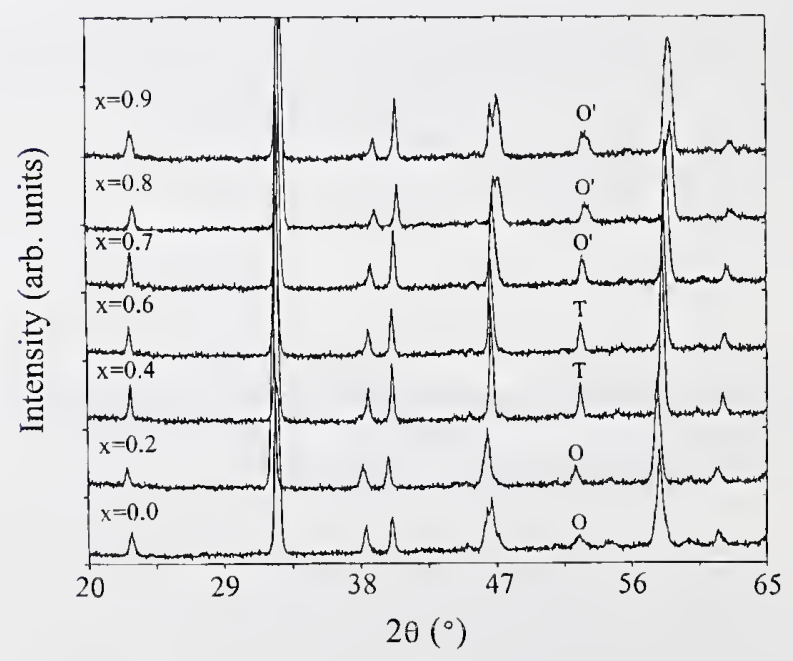

Fig. 2. X-ray patterns of $\mathrm{Ba}_{2-x} \mathrm{Nd}_{1+x} \mathrm{Cu}_{3} \mathrm{O}_{z}$ in $0.1 \% \mathrm{O}_{2}$ 
$(\mathrm{Ba} 2-\mathrm{xNd} x) \mathrm{CuO}_{3} \mathrm{z}$ and a nominally stoichiometric phase, $\mathrm{Ba}_{4} \mathrm{Nd}_{2} \mathrm{Cu}_{2} \mathrm{O}_{2}$, were confirmed. The latter is an insulator which consists of unusual 1-dimensional corner-sharing $\mathrm{CuO}$ s linear chains. In the boundary $\mathrm{BaO}-\mathrm{CuO} x$ system, two phases were found when the system was prepared in air, namely, $\mathrm{Ba}_{2} \mathrm{CuO}_{3}$ and $\mathrm{CuO}$; an additional $\mathrm{BaCu}_{2} \mathrm{O}_{2}$ phase was found in the diagram prepared under $0.1 \% \mathrm{O} 2$. Only $\mathrm{Nd}_{2} \mathrm{CuO}_{4}$ and $\mathrm{BaNd}_{2} \mathrm{O}_{4}$ were found in the $\mathrm{Nd}_{2} \mathrm{O}_{3}-\mathrm{CuOx}$ and $\mathrm{BaO}-\mathrm{Nd}_{2} \mathrm{O}_{3}$ systems, respectively. The structure of $\mathrm{BaNd}_{2} \mathrm{O}_{4}$ was studied by neutron diffraction using the Rietveld method.

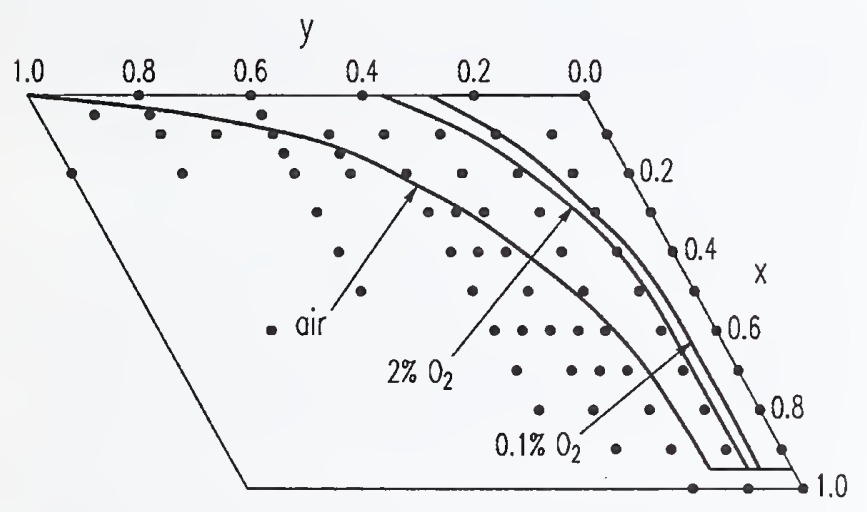

Fig. 3. Single phase regions of $\mathrm{Ba} 2-x\left(\mathrm{Nd}^{1}+x-y \mathrm{Yb}\right) \mathrm{Cu}_{3} \mathrm{O} 6+\mathrm{z}$

The range of the single-phase solid solution region for the phase $\mathrm{Ba} 2-\mathrm{x}\left(\mathrm{Nd}_{1+x-\mathrm{y}} \mathrm{Yb}_{\mathrm{y}}\right) \mathrm{Cu}_{3} \mathrm{O}_{6+z}$ has been determined as a function of oxygen partial pressure. Figure 3 shows a composite diagram of the single phase region (to the right of the curves) under $0.1 \% \mathrm{O}_{2}\left(810^{\circ} \mathrm{C}\right), 2 \% \mathrm{O} 2\left(810^{\circ} \mathrm{C}\right)$ and air $\left(930^{\circ} \mathrm{C}\right)$. In Fig. 3 , parallel to the $\mathrm{x}$ compositional axis, the samples have constant $\mathrm{Yb}$ content, while the $\mathrm{Ba}$ concentration changes as a result of substitution by $\mathrm{Nd}$. Parallel to the $\mathrm{y}$ compositional axis, $\mathrm{Yb}$ and $\mathrm{Nd}$ substitute for each other while the $\mathrm{Ba}$ is kept constant. The extent of the single-phase region decreases as the oxygen partial pressure decreases.
As $\mathrm{Nd}$ substitutes for $\mathrm{Ba}$, a sequence of phase transformations similar to that for the Nd-213 solid solution was determined. Neutron Rietveld refinements on selected compositions indicated that $\mathrm{Yb}$ substituted exclusively for $\mathrm{Nd}$ and not $\mathrm{Ba}$, while $\mathrm{Nd}$ substituted for $\mathrm{Ba}$. As Nd substituted on the Ba sites, the oxygen content of the samples increased, and oxygen and copper site disorder was also observed.

The onset melting temperatures decreased as $\mathrm{Yb}$ substituted for $\mathrm{Nd}$, and also as $\mathrm{Nd}$ substituted for $\mathrm{Ba}$. For example, the initial melting temperatures of the $\mathrm{Ba}_{2}-\mathrm{x}(\mathrm{Nd} 1+\mathrm{x}-\mathrm{y} \mathrm{Yby}) \mathrm{Cu}_{3} \mathrm{O}_{6+z}$ series with $\mathrm{y}=0.1$ decreased from $1080^{\circ} \mathrm{C}$ for $\mathrm{x}=0.1$ to $1058^{\circ} \mathrm{C}$ for $\mathrm{x}=0.7$. The onset $\mathrm{Tc}$ value decreased as the annealing oxygen partial pressure increase $\mathrm{d}_{3+}$ and as the amount of $\mathrm{Nd}$ increased. Substitution of $\mathrm{Nd}^{3+}$ on $\mathrm{Ba}^{2+}$ is thought to cause progressive reduction of the hole concentration on the $\mathrm{CuO}$ plane.

The structural, melting and superconductivity data discussed above are critical for processing and for tailoring flux-pinned materials. Future research on Ba-R-Cu-O phase equilibria will focus on the $\mathrm{R}=\mathrm{Y}, \mathrm{Sm}$ systems, and on the $\mathrm{Nd} / \mathrm{Y}$ mixed lanthanide system under reduced oxygen pressures.

\section{For More Information}

1. Wong-Ng, W., Cook, L.P.,. Suh,J., Coutts,R., Levin, I., Stalick, J. "Phase Equilibria Diagrams of the $\mathrm{BaO}-\mathrm{Nd}_{2} \mathrm{O}_{3}-\mathrm{CuO}$ system in Purified Air and $0.1 \% \mathrm{O}_{2}$ " to be submitted to J. Solid State Chem.

2. Wong-Ng, W., Swartzendruber, L., Kaduk, J.A., Bennett, L.H., "Magnetic and Structural Properties of the 'Brown Phase' Solid Solution Ba(Nd2-xLax)CuOs", submitted to J. Mag. and Mag. Mater.

Contact persons: Winnie Wong-Ng and Lawrence Cook 


\section{Polymer Chain Relaxation: Surface Outpaces Bulk}

The time scale that controls polymer chain relaxation has taken on a new importance as the paradigm for polymer innovation evolves from three dimensions to two. Nanotechnology initiatives, involving the design and fabrication of highly confined polymer layers, drive the need for a comprehensive description of chain dynamics, as bulk polymers become thinner and more surface like. In this study we apply near-edge X-ray absorption fine structure (NEXAFS) to measure directly both surface and bulk segmental relaxation throughout a uniformly deformed polystyrene slab. Using this methodology, in a single experiment, chain relaxation is found to occur almost $50 \%$ faster at the surface than in the bulk.

Rectangular samples $(12.5 \mathrm{~mm} \times 12.5 \mathrm{~mm} \times 6 \mathrm{~mm})$, prepared by vacuum hot-pressing at $150^{\circ} \mathrm{C}$ monodisperse polystyrene $\left(\mathrm{M}_{\mathrm{W}}=228,000 \mathrm{~g} / \mathrm{mol}\right)$, were placed into a steel channel die and uniaxially elongated at room temperature along one of the long dimensions to about $130 \%$ of their original length. This sample preparation allowed us to examine true bulk samples as encountered in practice and to avoid thin film effects that may alter the free surface behavior. Partial electron yield (PEY) and fluorescence yield (FY) carbon K-edge NEXAFS intensities, with probing depths of approximately $2 \mathrm{~nm}$ and $200 \mathrm{~nm}$, respectively, were recorded simultaneously at the NIST/Dow Soft X-ray Materials Characterization end-station (National Synchrotron Light Source, Brookhaven National Laboratory). ${ }^{3}$

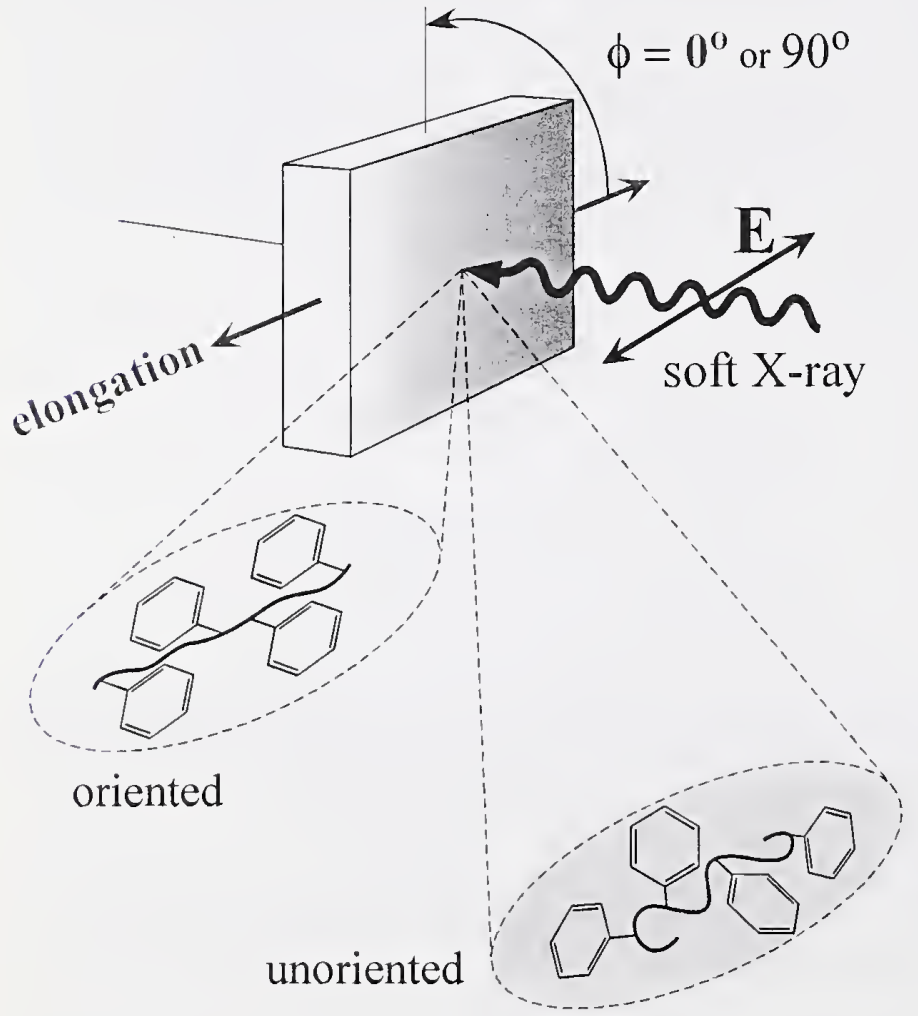

For each sample, PEY and FY were measured with the incident polarized X-ray beam normal to the sample surface and at two azimuthal sample orientations: with the electric field vector, $\mathbf{E}$, parallel $\left(\phi=0^{\circ}\right)$ and perpendicular $\left(\phi=90^{\circ}\right)$ to the elongation direction as shown in the figure to the left.

Orientation of the chain backbone was determined by monitoring the $\mathrm{C}=\mathrm{C}$ phenyl ring NEXAFS resonance intensity at $285.5 \mathrm{eV}$, which involves the excitation of carbon 1s electrons to the unfilled $\mathrm{C}=\mathrm{C}$ antibonding orbitals of the phenyl ring. Enhancement of the $\mathrm{C}=\mathrm{C}$ resonance intensity was observed when $\mathbf{E}$ was parallel to the elongation direction.

Since the phenyl $\mathrm{C}=\mathrm{C}$ orbitals are oriented normal to the phenyl rings, and the phenyl rings, free to rotate around the pendant bond, will have a component normal to the chain axis, the intensity of the $\mathrm{C}=\mathrm{C}$ signal has been shown to be an unambiguous signature of backbone orientation. This orientation, seen both in the PEY and the FY NEXAFS signals of the elongated samples, provides clear evidence of chain orientation at the outset of the experiment. A direct measure of the chain relaxation rates at the surface and in the bulk can be obtained by defining an orientation factor, OF (see fig. caption next page) that is evaluated from the time dependence of the $\mathrm{C}=\mathrm{C}$ resonance intensity in the PEY (relative uncertainty \pm $0.01 \%$ ) and FY (relative uncertainty $\pm 0.05 \%$ ) NEXAFS spectra, respectively, during annealing.

The schematic to the left details the sample geometry for the incident polarized soft X-ray beam normal to the polystyrene sample surface with the elongation direction parallel to $\mathbf{E}$ $\left(\phi=0^{\circ}\right)$. The insets in the lower right represent chain configurations in oriented (before annealing) and unoriented (after annealing) elongated samples. 
The graph shows that while the OF for both surface and bulk chains decays as a function of increasing annealing time at $60{ }^{\circ} \mathrm{C}$, the surface orientation is initially greater and decays faster than for the bulk. Fitting the decay rates to exponential functions gives characteristic time constants of approximately $33 \mathrm{~min}$ and $50 \mathrm{~min}$ (with corresponding $\mathrm{R}^{2}$ values of 0.90 and 0.91 ) for the surface and the bulk, respectively.

These results show conclusively that polystyrene surface chain relaxation dynamics are significantly faster than the bulk. Our results are in accord with recent theory predicting that collective motion on chain loops extended to the sample surface is responsible for a rapid increase of chain mobility near the surface region of the film (top $\approx 5 \mathrm{~nm}$ ). The finding that the polystyrene surface segmental mobility greatly outpaces the bulk is expected to be a universal property of polymeric chains profoundly influencing the design, processing, and application of polymeric materials.

Figure right: Tme evolution of the orientation factor, $\mathrm{OF}$, from an elongated (oriented) polystyrene sample reveals that when annealed at $60^{\circ} \mathrm{C}$ the surface chains relax to an equilibrium (unoriented) configuration faster than the bulk chains. OF is calculated from $(\mathrm{I} i \mid-\mathrm{I} \perp) /(\mathrm{I} \|+\mathrm{I} \perp)$, where $\mathrm{I} \|$ and $I \perp$ are the $\mathrm{C}=\mathrm{C}$ resonance NEXAFS intensities collected with the sample elongation direction parallel $\left(\phi=0^{\circ}\right)$ and perpendicular $\left(\phi=90^{\circ}\right)$ to the electric vector of the soft X-ray beam, $\mathbf{E}$, respectively. The blue circles denote $\mathrm{OF}$ from the partial electron yield NEXAFS signal (surface region) and the red circles represent the fluorescence yield NEXAFS signal (bulk region), measured simultaneously. The closed and open circles depict OF evaluated from data collected on elongated specimens and those not subjected to elongation, respectively. The solid lines represent exponential decay fits to the experimental data on the elongated samples; the rate decay constants are reported in the text.

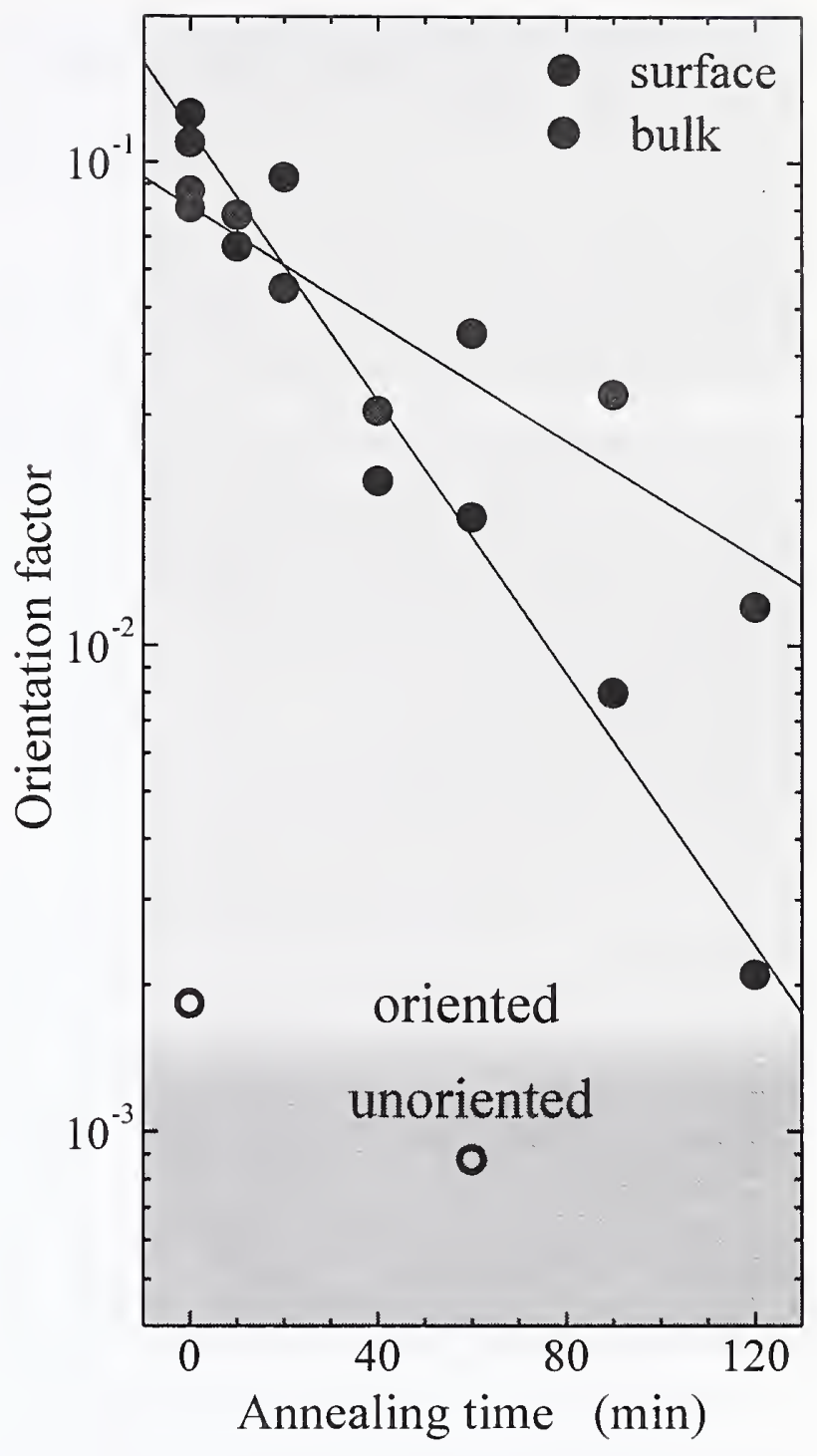

\section{For More Information $\quad$ D. A. Fischer}

W. E. Wallace, W. L. Wu (Polymers Divison)

On This Topic K. Efimenko, J. Genzer (NCSU) 


\section{Spatially Resolved Valence Electronic Structure by X-ray Standing Waves and Photoelectron}

\section{Spectroscopy}

We have developed a new experimental method by which site specific valence electronic structure may be obtained. It utilizes the spatial variation of the electric-field intensity that occurs near a crystal x-ray Bragg reflection in conjunction with high-resolution valence-photoelectron spectroscopy to determine the partial densities of occupied valence states of crystalline materials. Resolution of the anion and cation contributions to the GaAs valence band is demonstrated and compared to an ab initio theoretical calculation of the Ga and As partial density of states.

One of the most powerful experimental tools for examining the electronic structure of a solid or film is photoelectron spectroscopy. Due to the conservation of energy between the incident photon and the ejected photoelectron, direct and important electronic information pertaining to the occupied valence-band density of states has been obtained for many materials. This information has been used to establish the validity of complicated band-structure calculations for metals, semiconductors, insulators, and alloys.

Typical photoemission measurements are performed with excitation sources that are monochromatic plane waves. As the intensity of a plane wave is constant over the dimensions of the crystalline-unit cell, standard photoemission measurements are unable to produce direct, site-specific valence information. However, such information is important for advancing our understanding of how chemical bonding results in the solidstate electronic structure.

Here we describe a new experimental method for obtaining site-specific valence-electronic structure. It utilizes the spatial variation of the electric-field intensity that occurs within the vicinity of a crystal $\mathrm{x}$-ray Bragg reflection which is given by the superposition of the incident $\mathbf{E}_{\mathrm{O}}$ and reflected $\mathbb{E}_{\mathrm{h}} \mathrm{X}$-ray beams that travel with wave vectors $\mathbf{k}_{\mathbf{O}}$ and $\mathbf{k}_{\mathrm{h}}$, polarization vectors $\mathrm{e}_{\mathrm{o}}$ and $\mathrm{e}_{\mathrm{h}}$, and frequency $\omega$ :

$E(r, t)=\left[e_{o} E_{0} e^{i k_{o} r}+e_{h} E_{h} e^{i k_{h}} r^{r}\right] e^{-i \omega t}$. $\mathbf{k}_{\mathrm{O}}$ and $\mathbf{k}_{\mathbf{h}}$ are connected by the Bragg condition $\mathbf{h}=\mathbf{k}_{\mathrm{O}}-\mathbf{k}_{\mathbf{h}}$, where $\mathbf{h}$ is a reciprocal-lattice vector of the crystal. For the $\sigma$ polarization geometry of a symmetric reflection, this field squares to give the electric-field intensity at an arbitrary point $\mathbf{r}$ in space:

$I(\mathbf{r})=\left|E_{0}\right|^{2}[1+R+2 \sqrt{R} \cos (v+\mathbf{h} \cdot \mathbf{r})]$.

$v$ is the phase of the complex-field amplitude ratio $\mathrm{E}_{\mathbf{h}} / \mathrm{E}_{\mathbf{O}}=$ $\sqrt{R e^{i v}}$, and $R$ is the reflectivity function $R=\left|E_{h} / E_{O}\right|^{2}$.

Germane to the method is the unique ability to position the maxima (or minima) of the electric-field intensity at any location within the crystalline-unit cell by experimentally varying the phase of the complex reflectivity function between $0<\nu<\pi$. This is achieved by slightly varying either the sample angle or the photon energy within the natural width of the crystal x-ray Bragg reflection.
Because the probability of emission of an electron from an atom in an external electric field is proportional to the electricfield intensity at the location of its atomic core (dipole approximation), by selectively positioning the electric-field intensity within the crystalline-unit cell and recording highresolution valence-photoelectron spectra, spatially resolved components to the photoemission valence-band density of states may be directly obtained.

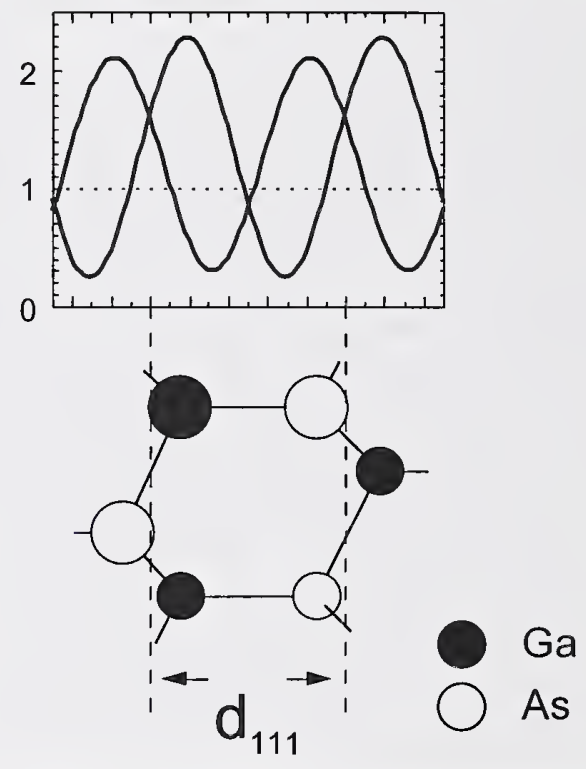

Figure 1: Theoretical calculation of the electric-field intensities and their spatial dependencies for different $v$.

To illustrate the principal of the technique, Figure 1 shows a theoretical calculation of the electric-field intensities and their spatial dependence relative to the GaAs atomic planes for different values of $v$ within the photon-energy range of the GaAs(111) Bragg back-reflection condition. Significant contrast between the $\mathrm{Ga}$ and As contributions to both the core and valence photoelectron-emission spectra can therefore be obtained for this heteropolar zinc-blende crystal by simply experimentally varying the phase $v$ as demonstrated by Figure 2. The data in Figure 2 exhibit the well known $x$-ray standing wave effect for both the Ga $3 \mathrm{~d}$ and As $3 \mathrm{~d}$ core levels, as well as for the crystal valence band. Note either the enhancement or suppression of the relevant emission depending on which of the atomic planes (either Ga or As) was preferentially excited. 


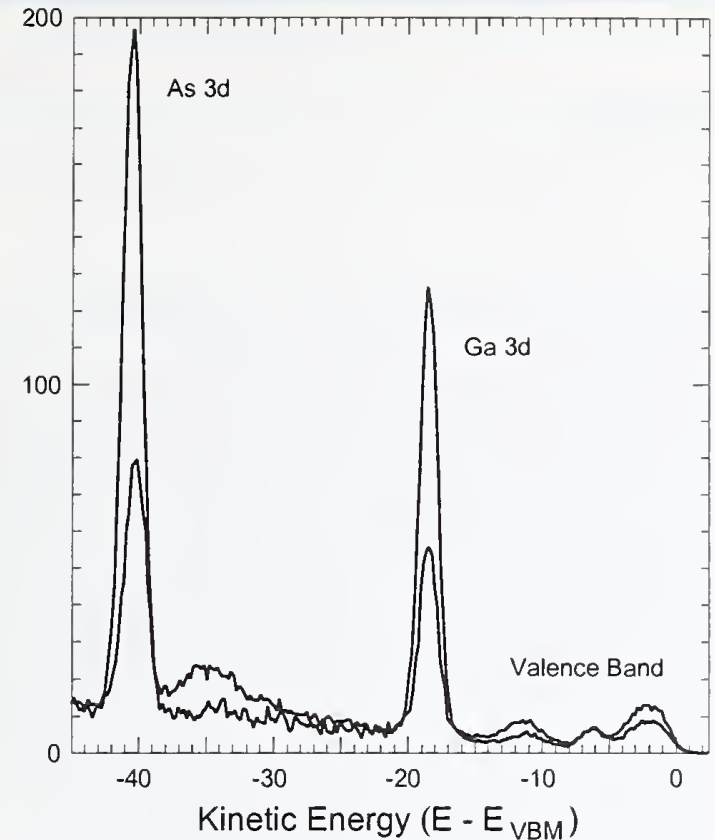

Figure 2: Photoemission spectra recorded from crystalline GaAs under the different electric-field conditions of Fig. 1.

In order to uniquely resolve the individual contributions to the GaAs valence band, we proceed as follows. Under condition of constant electric-field intensity, the valence photocurrent can be approximated as the linear sum of the individual partial-density of states $\rho_{i}(E)$ arising from the different $i$ atoms of the unit cell weighted by the energy dependent, relative photoionization cross sections $\sigma_{i}(E, h \omega)$ of each of the states:

$\mathrm{I}(\mathrm{E}, \mathrm{h} \omega) \alpha \Sigma_{\mathrm{i}} \rho_{\mathrm{i}}(\mathrm{E}) \sigma_{\mathrm{i}}(\mathrm{E}, \mathrm{h} \omega)$.

For the case of a spatially dependent x-ray interference field, this expression must be modified to include the electric-field intensities at each of the different core sites:

$\mathrm{I}(\mathrm{E}, \mathrm{h} \omega) \alpha \Sigma_{\mathrm{i}} \rho_{\mathrm{i}}(\mathrm{E}) \sigma_{\mathbf{i}}(\mathrm{E}, \mathrm{h} \omega)\left[1+\mathrm{R}+2 \sqrt{\mathrm{R}} \cos \left(v+\mathbf{h} \cdot \mathbf{r}_{\mathbf{i}}\right)\right]$.

Consequently, for a two atom unit cell such as GaAs, all that is necessary to uniquely resolve the individual chemical components of the GaAs valence band is to obtain valence spectra at two different electric field conditions and then solve a simple set of two linear equations.

Figure 3 shows the resulting chemically resolved components of the GaAs valence band obtained by taking the appropriate linear combinations of the spectra from figure 2 . These components are compared to an $a b$ initio theoretical calculation of the Ga and As partial-density of states computed by Professor James Chelikowsky's group at the University of Minnesota by using ab initio pseudopotentials within density functional theory with a plane-wave basis.

The site-specific density of states curves were computed by using a sphere corresponding to the Ga-As covalent radius centered on each atom to deconvolve the obtained wavefunctions over atomic orbitals of valence electrons.

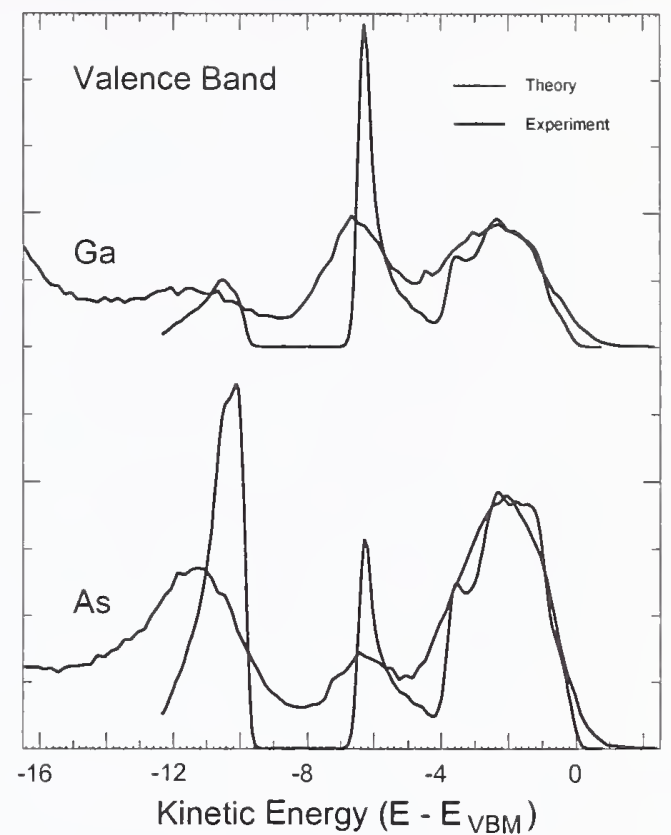

Figure 3: Comparison of the $\mathrm{Ga}$ and As partial density of states curves from both theory and experiment.

The calculations clearly show the differences between the two electronic structures centered around each atomic core. These differences occur due to the natural ordering of the Ga and As atomic $4 s$ and $4 p$ valence states, coupled with the solid-state bonding that has occurred between them and may be directly correlated with the crystalline band structure. Remarkable qualitative agreement between theory and experiment is observed, even though experimental resolution and cross-section effects have not been considered theoretically.

In conclusion, we have demonstrated that valencephotoelectron spectra recorded in the vicinity of a crystal $\mathrm{x}$ ray Bragg reflection can reveal site-specific valence information that is directly related to the partial density of occupied valence states. It is our hope that this method will provide useful information pertaining to the detailed electronic structure of more complex crystalline materials that are currently of interest to the scientific community.
For More Information On This Topic
J.C. Woicik, E.J. Nelson, T. Kendelewicz, P. Pianetta, M. Jain, L. Kronik, and J.R. Chelikowsky, in press, Phys. Rev. B, Rapid Communications. J.C. Woicik, E.J. Nelson, and P. Pianetta, Phys. Rev. Lett. 84, 773 (2000).

J.C. Woicik and E.J. Nelson 



\section{Ceramic Coatings}

The Ceramic Coatings Program addresses plasma spray deposited and physical vapor deposited ceramic thermal barrier coatings (TBCs) used in aircraft, land-based turbines, and diesel engines as well as wear resistant coatings used in many applications. These materials are a significant portion of the nearly one billion dollar North American ceramic coatings market. A primary goal of this program is improve the reliability of ceramic coatings. Collaborations have been established, e.g. Pratt and Whitney, General Electric, Caterpillar, METCO, Praxair Coating Technologies, as well as the Thermal Spray Laboratory at the State University of New York at Stony Brook, NASA Lewis Research Center and the Thermal Spray Laboratory at Sandia National Laboratory to enable research on relevant materials and to transfer results to users. Collaborations are also underway with Bundesanstalt für Materialfurschung und -prufung) (BAM) and Deutsche Forschungsanstalt für Luft-und Raumfahrt (DLR), both in Germany, for the development of characterization techniques for thin, hard coatings and TBCs. A strong attribute of the coatings research program is the use of common materials for which complementary data can provide a more complete understanding of processingmicrostructure-property relationships.

Participants in the NIST program are located in MSEL, i.e., Ceramics Division, Materials Reliability Division, Metallurgy Division, and the NIST Center for Neutron Research, as well as in the Chemical Science and Technology Laboratory.

The program has the following elements:

- Development of predictive models for the long-term reliability of ceramic coatings under operating conditions.

- Relating microstructural characteristics such as fine voids and phase stability to thermal and mechanical properties.

- Developing and validating microstructure based models that predict coating performance.

- Development of measurement methods, such as online instrumentation, that enable improved control of thermal spray processes. 


\section{Certification of WC/Co}

Particle Size Distribution Reference Materials
Feedstock size distribution is a primary parameter controlled in thermal spray deposition of coatings. We have developed standard reference materials for calibration of particle size distribution measurement instruments important in the production of thermal barrier coatings and wear resistant coatings.

\section{James F. Kelly and Patrick Pei}

At the 1992 NIST/Industry Workshop on Thermal Spray, powder characterization and quality were identified as important issues with a major impact on the manufacture of cost-effective coatings. The development of repeatable and reproducible measurement methods especially for particle size distribution (PSD) requires the use of standard reference materials. The two important powders identified at the workshop were yttria stabilized zirconia (YSZ) for thermal barrier coatings and tungsten carbide/cobalt $(\mathrm{WC} / \mathrm{Co})$ for hard abrasion resistant coatings. SRM 1982 for YSZ thermal spray powder was issued in 1996 and the WC-Co reference materials SRM 1984 and SRM 1985 were certified this year as particle size distribution standards. These represent the two major WC powder types used by that industry.

Two WC/Co powders were purchased from OSRAM Sylvania Products Inc. , Towanda, PA. These powders were manufactured by spray drying an aqueous mixture of tungsten carbide and cobalt powders. The spray dried powder was sintered and plasma spray densified. The acicular material used for SRM 1984 (Figure 1) was crushed before final screening to obtain the desired size distribution. The material for SRM1985 was not crushed and retains a spheroidal shape. These powders were selected for their size distribution, resistance to fracture on handling, and low level of aggregation.

Test bottles were analyzed by laser light scattering and SEM. Particle size distributions describing the percentage of powder volume represented by particles with diameters less than a given length were calculated using the weighting factors obtained from the sieving results. The size distribution certification for the thermal spray materials also included round robin studies using laser light scattering methods with industrial participants from powder users, producers and instrument and equipment manufacturers. A graphical comparison of the distributions measured by SEM with the mean distributions obtained by laser light scattering is shown in the Figure for SRM 1984.

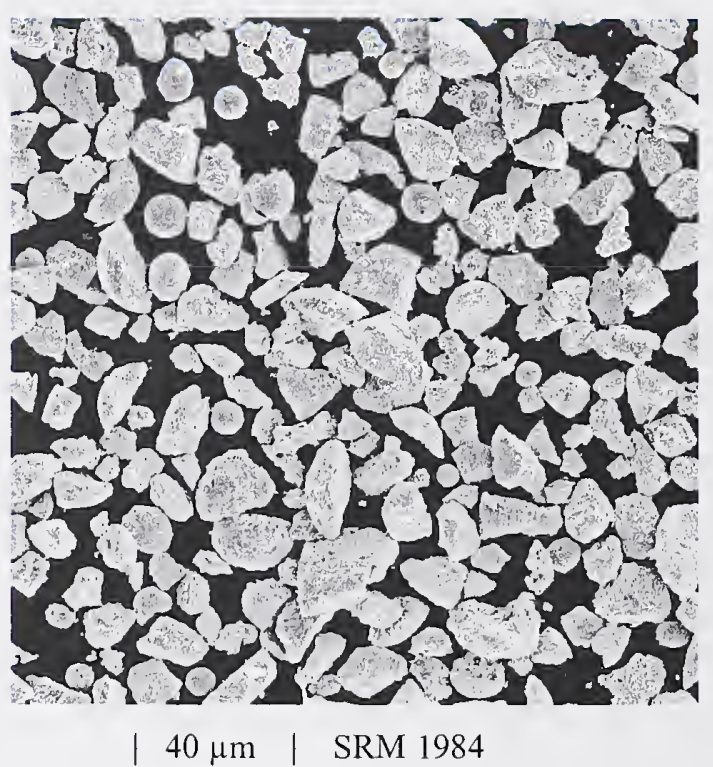

(A)

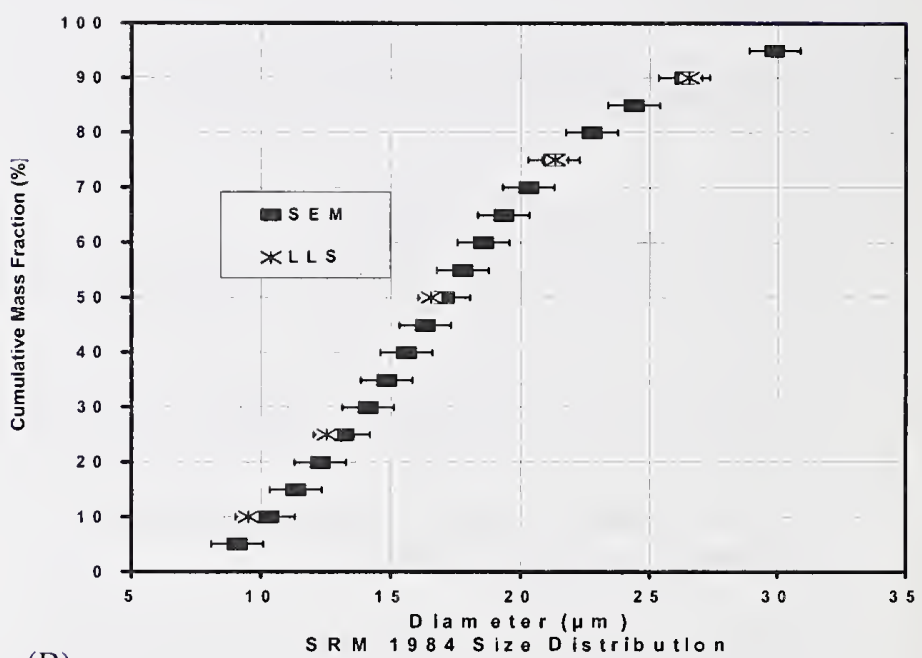

(B)

Fig. 1 Morphology (A) and Size Distribution (B) of SRM 1984, WC/Co for Thermal Spray
Contributors and Collaborators
OSRAM Sylvania, Pennsylvania State University, Sympatec Inc., Dirats Laboratory, Stellite Coatings, Honeywell Microtrac, Horiba Instruments Inc., Duke Scientific Corp. and Beckman-Coulter 


\section{Instrumented Indentation}

\author{
Douglas T. Smith
}

Instrumented indentation is an effective method of characterizing the mechanical properties of thin films and coatings, but its value in coating development and product specifications is limited by the fact that no national or international standard test methods for its use exist. This program seeks to develop standard test methods and materials (SRM's) both for the performance of the test and for the analysis and interpretation of the test results.
This project deals with the use of instrumented indentation to probe the mechanical properties of coatings and thin films. The indentation machines at NIST permit studies at peak indentation loads ranging from $40 \mu \mathrm{N}$ to over $20 \mathrm{~N}$, using Vickers, Berkovich and spherical diamond and WC-Co indenter tips. The resulting experimental load-displacement curves are analyzed to yield the hardness and Young's modulus of the material probed, as well as the energy absorbed in the indentation process. Since elastic modulus measurements are sensitive to discontinuities in the microstructure, such as cracks and crack-like voids, they are well suited to the study of changes that occur as the result of changing fabrication conditions or post-fabrication treatments. Relatively small volumes of material are probed in each test, permitting local mapping of mechanical property variations and the determination of hardness and elastic modulus for thin films as well as coatings.

The focus of the project is on the development of the technique of instrumented indentation, rather than the application of the technique to particular material systems, although data are taken on specific materials of technological interest (e.g., thermal barrier and wear-resistant coatings), as well as candidates for Standard Reference Materials. International workshops, symposia, and round robin standards tests are organized and executed in an effort to guide the instrumented indentation community toward greater standardization in data analysis, to the expand the range of mechanical property characterization possible with the technique, and to develop physical standards for the technique. In the past year, significant progress has been made within ASTM and ISO on the standardization of the test method. Although these standards deal initially only with the study of bulk materials, they lay the groundwork for later application to coating and thin film testing. NIST is a co-organizer of a large international round robin (VAMAS TWA 22, Project 1), now nearly completed, to study the application of the technique to thin films.

In an unusual application of the technique, studies were done on a series of thick (several $\mathrm{mm}$ ) functionally graded thermal barrier coatings prepared at the Materials Engineering Laboratory in Tsukuba, Japan, by a spark plasma sintering process. The coating/substrate system consisted of a pure 410 stainless steel base, a pure yttriastabilized zirconia top coat and nine intermediate layers of mixed composition to transition smoothly from base to top coat. Polished cross-sections of the coating were indented to measure hardness and Young's modulus through the coating thickness. The results, Figure 1 beloww, showed that although the hardness increased monotonically with zirconia content, the modulus of several of the intermediate layers was anomalously low. This was later shown to be due to insufficient sintering in those layers, a problem that was not recognized prior to the indentation work.

Figure 1: Hardness and modulus through the thickness (axial position) of a graded stainless steel/zirconia coating, for several distances from the central axis of the specimen.
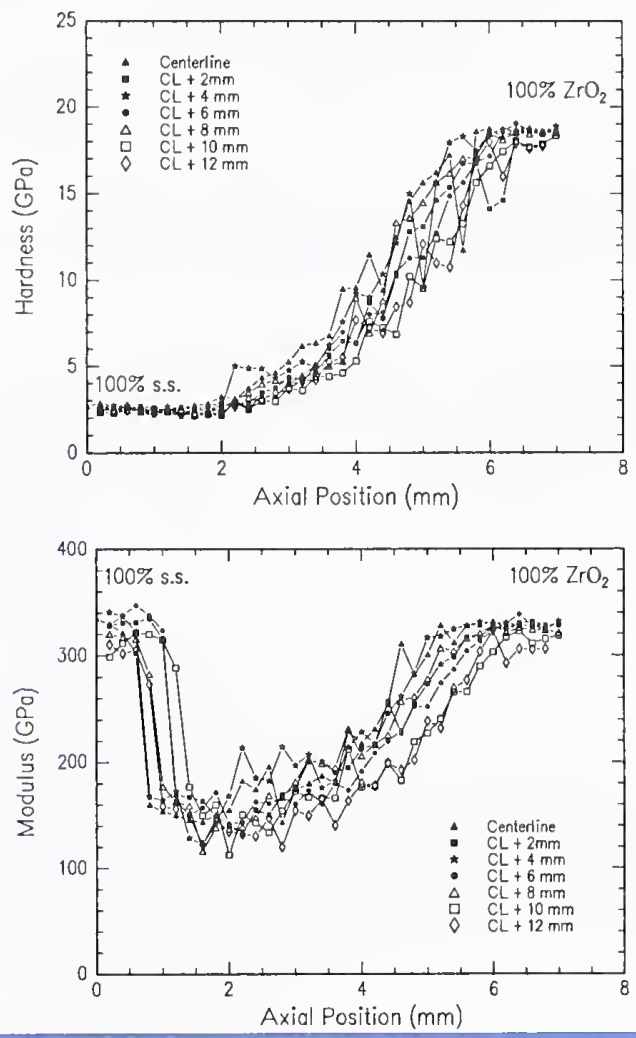

Contributors and Collaborators
Jay Wallace, Ceramics Division, NIST

Donna Hurley, Materials Reliability Division, NIST

Stuart Saunders and Nigel Jennett, National Physical Laboratory, U.K.

Toyonobu Yoshida, University of Tokyo, Japan

Yuji Enomoto, Mechanical Engineering Laboratory, Tsukuba, Japan 


\section{Reliability Prediction}

\section{and Modeling}

Thermal barrier coatings (TBC's) are used on superalloys in aircraft engines and industrial gas turbines as thermal insulation and oxidation barriers. In these applications, the $T B C$ must be able to withstand elevated temperature exposure and thermal cycling. TBC failure occurs when the coating delaminates from the surface over tine due to progressive damage accumulation near the alloy-coating interface. Accordingly, this research seeks to provide insights into the mechanisms that cause TBC failure and to develop predictive models for the long-term reliability of ceramic coatings under operating conditions.
Air-plasma sprayed (APS) thermal barrier coatings (TBC's) are typically a three-layer structure. A metallic bond coat layer, such as a Ni-Co-Cr-Al-Y alloy is applied to the underlying nickel-based superalloy to prevent its oxidation. A ceramic top coat layer, which is usually yttria-stabilized zirconia (YSZ), is used for thermal protection of the alloy. Finally, at elevated temperatures oxygen penetrates the YSZ top coat to form a thermally grown oxide (TGO) between the top coat and the bond coat. The TGO is primarily a-alumina, which forms from the $\mathrm{Al}$ in the bond coat, but other oxides, such as nickel-aluminum spinel, $\mathrm{NiAl}_{2} \mathrm{O}_{4}$, or yttrium aluminum garnet, $\mathrm{Y}_{3} \mathrm{Al}_{5} \mathrm{O}_{12}$, occasionally form. Thickness of the oxide increases over time, affecting spalling or delamination of the top coat. This behavior, however, is strongly influenced by interfacial roughness between the bond coat and the top coat. When a coating is cooled from the operating temperature, this roughness and the differing thermal contraction between the superalloy substrate, the metallic bond coat, the ceramic top coat, and the TGO results in tensile residual stresses normal to the interface, which cause delamination cracking. Interface roughness, while detrimental to reliability, is usually necessary to enhance splat adhesion during the spray deposition of the top coat.

Various phenomenological relationships have been developed in industry to predict spallation life, but few are mechanism based. Accordingly, microstructural parameters controlling reliability are poorly understood. This research develops a fracture-mechanics-based model for crack growth and damage evolution in the top coat above a rough interface. Finite element analysis is used to elucidate the influence of various microstructural parameters, including interfacial roughness and TGO growth, on the residual stress state in the top coat on cooling, and accordingly, damage evolution.

A simple model of periodic asperities with constant curvature was constructed to parameterize surface roughness. Three parameters describe the interface geometry: the asperity wavelength, the peak-to-valley amplitude, and the ratio of the radius of curvature of the peaks to that of the valleys. Virtual experiments were preformed on these simulated micro- structures using the Object Oriented Finite Element (OOF) analysis software developed at NIST. Residual stresses after cooling were determined at different stages of TGO growth and used to elucidate which interfacial roughness parameters had a significant influence on the residual stress state. A factorial design with 12 experimental microstructures was used to identify the important roughness parameters, namely, the peak-to-valley amplitude and the asperity radius-ofcurvature ratio. Asperity wavelength was mainly a scaling variable for the horizontal length scale. TGO growth had a major affect on the residual stresses normal to the interface. When the TGO thickness was below a critical thickness the normal residual stress above the peak of an asperity was tensile. However, when the TGO thickness exceeded this critical value, these stresses became compressive. When this occurred, the tensile stresses in the top coat moved down from the asperity peaks into the valley region.

A fracture mechanics model was used to analyze how the stress state affects crack extension in the top coat as a function of TGO growth and thermal cycling. Initially the crack extension is stable until a critical TGO thickness, when unstable growth gives a crack of sufficient size to cause a buckling delamination, or TBC failure. The fracture mechanics model uses a weight function approach to calculate the stress intensity factors for the two stress states: tensile stresses above asperity peaks and compressive stresses above asperity peaks. The first configuration is straightforward, leading to a stable crack configuration propagating horizontally above the asperity. However, after the stress state at the asperity switches, the compressive stresses cause closure over the central portion of the crack. A new stratagem was developed to handle these crack closure stresses. The net effect is to enhance the stress intensity factor at the crack tip, such that the crack can become unstable under certain conditions. Further work is exploring the influence of top coat modulus, bond coat plasticity, and TGO growth stresses on this phenomenon.
Contributors and Collaborators
Ravi Kacker, Montgomery Blair High School, Silver Spring, MD

James Ruud and Curtis A. Johnson, General Electric Corporate Research and Development, Schenectady, NY

Stanley J. Dapkunas, Ceramics Division (852), MSEL 
Stress Measurements in Thermally Cycled Thermal Barrier Coatings
Thermal barrier coatings are widely used in power generation and transportation engine applications to achieve higher engine efficiency by operating at elevated temperatures. Coating spallation, leaving the bare metal substrate exposed to high temperature, is a major concern. Spallation has been associated with stresses generated in an oxide layer formed during exposure of the coated components to elevated temperature, but the stress evolution and failure mechanisms are not understood. We are measuring these stresses and relating them to microstructure evolution in the oxide layer as a function of thermal exposure.
We are measuring stress evolution in the thermally grown oxide (TGO) that forms in thermal barrier coating (TBC) systems at elevated temperature. The TGO forms between the $\mathrm{TBC}$ and the metallic bond coat and forms an oxidation barrier that protects the metallic substrate from chemical attack. Stresses form in the TGO as it grows, and the eventual spallation of the coatings has been attributed to these stresses. However, the evolution of stress with time and the dependence of the stress on microstructural changes that lead to spallation is not understood.

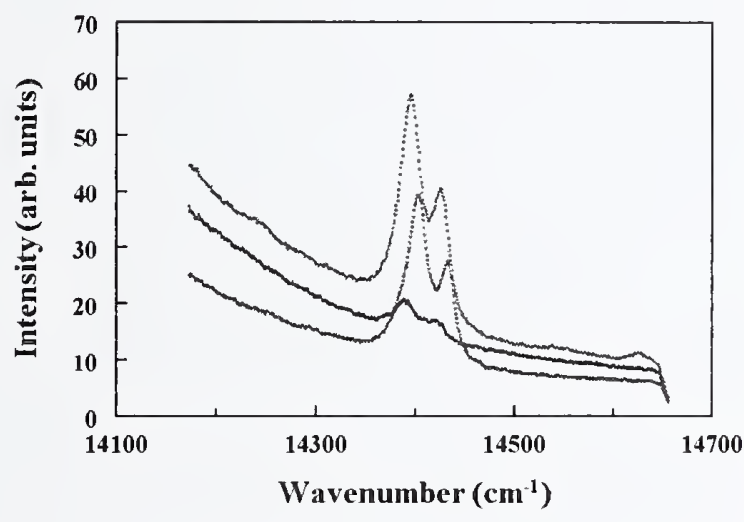

Figure 1: Photoluminescence spectra of the alumina TGO in a plasma sprayed TBC. Differences in peak positions of the three curves reflect different stress states of the TGO.

We are using photoluminescence $(\mathrm{PL})$ of the alumina TGO to monitor the value of the stress tensor as a function of thermal cycles (Figure 1). Our PL data indicate that, after one thermal cycle to $1150^{\circ} \mathrm{C}$, there was a wide range of compressive stress in the alumina (Figure 2). As the number of cycles increased, the range of stress values narrowed substantially. We were surprised to see that the median stress value remained nearly constant during this thermal treatment. After 350 thermal cycles, the coatings completely spalled from the substrates at room temperature, accounting for the stress relief seen in Figure 2.

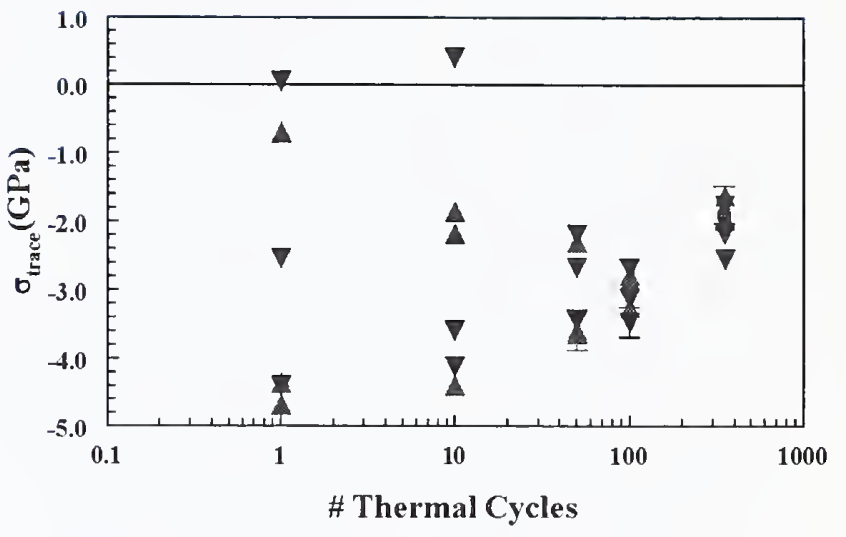

Figure 2: Trace of the stress tensor in the TGO as a function of numbers of thermal cycles. Two specimens were measured after each period of thermal cycles. The wide range of data reflects widely varying stress values from point to point.

Measurements made on the same specimens several weeks after thermal treatment indicate that substantial stress relaxation had occurred (i.e., stress values were still compressive, but were nearer to zero). Micrographs of the TGO show extensive microcracking had occurred. The microcracking was particularly severe for the specimens that had undergone 100 thermal cycles, and the cracks extended into the adjacent TBC.

In contrast, measurements made at room temperature as a function of time after $100 \mathrm{~h}$ exposure to $1150^{\circ} \mathrm{C}$ but without cycling did not exhibit this stress relaxation. The TBC specimens used in this latter set of experiments were obtained from a different source, although the composition was very similar to the first set of specimens. We are in the process of comparing the microstructure of both sets of specimens to try to understand this difference in behavior.
Edwin Fuller, Jr., and Mark Locatelli, Ceramics Division, (NIST)

DoE and NASA were sources of specimens 
Thermal Property Prediction by Simulations
Gas turbine designers are using thermal barrier coatings (TBC's) for more critical applications in power generation and aircraft engines. Knowing basic physical properties of TBC's is essential for the thermo-mechanical design of components using these coatings. In particular, point-to-point knowledge of thermal conductivity is crucial in the design of advanced turbine airfoils to allow more precise part temperature and life assessment. Accordingly, this research seeks to develop, validate, and apply finite-element simulations for the prediction of TBC thermal conductivity from digital images of microstructures.
As thermal barrier coatings (TBC's) are used in more critical applications in advanced engines, extensive materials development effort in industry has been undertaken to produce more reliable and reproducible TBC's. Development has focused on the materials system triangle: Reliability (thermo-mechanical properties), Reproducibility (process control), and Performance (thermal properties). Of the three legs, however, optimization of TBC thermal performance is usually under-emphasized for two reasons. First, as TBC's were historically used to extend life of turbine components, ensuring coating reliability and reproducibility was more critical than optimizing thermal performance. Second, although a critical design parameter, thermal conductivity is difficult, costly, and time-consuming to measure. Equipment for such measurement is specialized and expensive, and few reliable vendors are able to provide them. As a result this key property has been under-emphasized. Extension and validation of the innovative object-oriented finite-element (OOF) software developed at NIST offers the possibility of providing industry with a rapid, inexpensive tool for determining the thermal conductivity from TBC microstructures.

Progress was made in three areas: development of a TBC thermal conductivity database, protocols for microstructure input into OOF, and extension of the OOF code to thermal conductivity.

General Electric Corporate Research and Development (GE CR\&D) selected three archived sets of TBC microstructures for thermal conductivity characterization: a dense (set A) and a porous (set B) microstructure, which were thermally aged under various conditions, and a range of as-deposited microstructures (set C). The three specimen sets, which were attained through variations in process conditions and thermal annealing, encompass a wide variation in TBC microstructure and conductivity, and accordingly, will be used for initial verification of the OOF thermal conductivity simulations. Thermal conductivities for all three sets were measured at room and elevated temperature using a laser-flash technique. The range of thermal conductivities measured for specimen set $C$ varied by a factor of 3 from the lowest $(0.53 \mathrm{~W} / \mathrm{m} \cdot \mathrm{K})$ to the highest $(1.66 \mathrm{~W} / \mathrm{m} \cdot \mathrm{K})$. However, this variation did not correlate with material density. As expected, it is not absolute porosity that determines thermal conductivity, but the detailed distribution of pores and microcracks, thus necessitating the use of a microstructural simulation approach, such as OOF, to predict thermal conductivity. These three specimen sets will be used for initial verification of the OOF thermal conductivity module. Based on these results, designed experiments will be planned with new specimens to validate the predictions.

As robust protocols are needed for inputting and accurately representing TBC microstructures, initial simulations were undertaken to calculated elastic properties. Because thermal conductivity and elastic modulus of porous, cracked materials are cross-correlated, we expect that input protocols that produces reliable elastic modulus predictions will also be robust for thermal conductivity simulations. A subset of specimen set $\mathrm{C}$ with known elastic moduli was used for the elastic property simulations. Effects of three factors on the modulus prediction were studied: 1) magnification of the image, 2) accuracy of the finite-element modulus calculations for ideal microstructures, and 3) accuracy of the meshing. To date, estimates of the elastic moduli via OOF have been substantially higher than measured values. However, modulus for simple ideal ellipsoidal pores in a dense body agreed well with analytical predictions. Causes for the overestimates of modulus are likely the inability of the image analysis and meshing techniques to represent accurately the fine interlamellar porosity and trans-splat microcracks. Work is underway to identify alternate image analysis procedures.

Finally, work to develop an OOF module for thermal conductivity simulations is proceeding along two fronts. Edwin Garcia and Steve Langer developed a preliminary version of OOF that can compute thermal conductivity by modifying a version that performed piezoelectricity calculations. This version is being tested and debugged by N.S. Hari at GE and by Steve Langer and Ed Fuller at NIST. Because the thermal and piezoelectric versions of OOF were created by adding code to the original elasticity version, they lack flexibility, ease-of-use, and a user-friendly interface design. Accordingly, work is underway on a new version of OOF that will be more easily applied to thermal conductivity problems. This version will be faster and smaller than the original, as well as be easier to use.
Contributors and Collaborators
James Ruud, N. S. Hari, and A. Mogro-Campero, General Electric Corporate Research and Development, Schenectady, NY; Edwin Garcia, Massachusetts Institute of Technology, Cambridge, MA; Mark R. Locatelli and Stanley J. Dapkunas, Ceramics Division (852), MSEL 


\section{Ceramic Manufacturing Program}

Most ceramic products are produced by powder processing, where raw material powders are mixed with forming additives and shaped by various means into green bodies, which are then fired to the final product. Depending on the intended application, parts may require machining, the costs of which can represent a significant fraction of the total manufacturing cost. Also, because ceramics are susceptible to brittle fracture, the manufacturing steps used in their production must be carefully controlled and/or monitored to ensure that the required properties are obtained. One key to reliable and costeffective development of new products is the availability of established test methods to analyze the material at its different stages of manufacturing.

The primary objective of the Ceramic Manufacturing Program is to provide measurement techniques, standards, basic data, and predictive models needed by U.S. industry for cost- effective manufacturing of reliable ceramic products. Research activities include off-line and on-line measurement methods needed for processing of ceramic powders and suspensions, and for machining processes applied to ceramics. The research activities are focused on the characterization of particles and of particle suspensions and rheological properties of ceramic slurries, identification of the influence of powder characteristics and processing conditions on the resultant microstructures, homogeneity measurements on pre-sintered ceramics, identification of machining induced damage and the influence of this damage on mechanical properties, and development of test methodologies and mechanistic models of mechanical behavior of ceramics which are required for reliable use in a variety of applications. The results of the research activities in the program are used to develop new measurement methods and Standard Reference Materials that are needed for calibration of measurement instruments used for key manufacturing steps and for reliability analysis. The reliability and precision of various measurement techniques and their suitability for standardization are assessed jointly with industrial partners, international measurement laboratories, and national and international standards organizations. The close working relationship developed between these organizations and NIST not only ensures the relevance of the research projects but also promotes an efficient and timely transfer of research information to industry for implementation. 


\section{Abrasive Finishing and Wear of Dental Ceramics}

The development and application of ceramic dental restorative materials requires knowledge of abrasive finishing response and wear characteristics to assess performance and reliability. This project develops and evaluates test methods to measure the abrasive finishing and wear behavior of dental restorative materials..

\section{Said Jahanmir and Lewis K. Ives}

The use of ceramics for dental restorations has increased significantly due primarily to their desirable aesthetics, and inherent biocompatibility. A number of quantities are needed to determine the suitability of a prospective material or existing material for a given dental application. Among these are fabricability and wear resistance. Abrasive finishing is nearly always used at some stage during the fabrication and fitting of a restoration. Abrasive finishing response can be characterized in terms of material removal rate, surface roughness, and degree of surface damage. To measure these quantities an instrumented test device using a conventional dental handpiece was designed and constructed. As an example of results obtained with this device, Fig. 1 shows the effect of different burr grit sizes on edge chipping for a glass-infiltrated alumina restorative material. Chipping is a

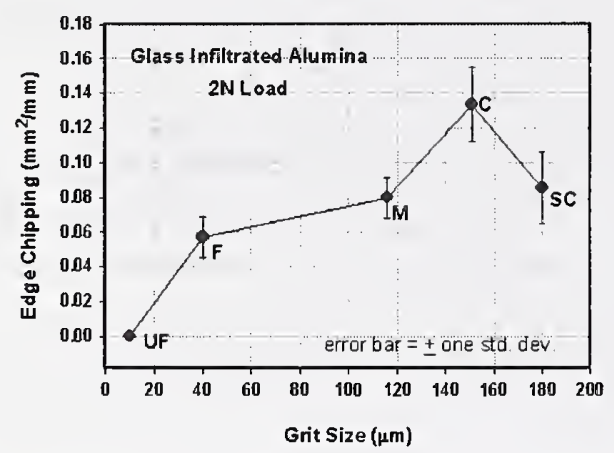

Fig. 1 Effect of bur grit size on edge chipping.

manifestation of the introduction of subsurface cracks, which can seriously degrade the performance and reliability of a restoration. For this material, it is likely that coarse-grit burrs will cause excessive damage that would have to be removed by a subsequent final-finishing step. This project has measured the abrasive finishing response of a number of important ceramic restorative materials and investigated the relationship between finishing response and materials properties and microstructure. These results will assist in the development of improved materials and better methodologies for preparing restorations.

Wear performance is a critical factor in both the development and application of dental restorative materials. Under a given set of conditions it is determined by materials properties and microstructure as well as by surface finish and machining damage. In general, the wear resistance of a restorative material should at least equal that of natural human enamel, but in any case, a material should not cause excessive wear to an opposing tooth. A number of test methods have been devised to assess the wear of dental materials. Although these test methods have shown some capacity to rank different materials, they have not been able to provide a reliable prediction of actual clinical performance. Work on this project has focussed primarily on an investigation of the relatively widely used pin-on-disk test. As is true for all laboratory tests, this test does not simulate directly the very complex tooth contact conditions in the human mouth. However, with careful control of the test parameters, limited but critical aspects of the contact can be evaluated. This requires a detailed knowledge of the possible influence of test parameters on wear mechanisms, extending beyond the usual approach of simply selecting a load and sliding distance. For example, Fig. 2 shows that for the dental glass-ceramic material used in the test, increasing the diameter of the disk wear track results in a substantial increase in wear rate. Careful microscopic examination of the wear tracks indicated that increasing the track diameter decreased the efficiency for formation of a protective compacted debris layer. In other project studies it was shown that contact pressure gave a better correlation with wear rate than applied load. Thus, greater consideration should be given to evaluating performance based on contact pressure.

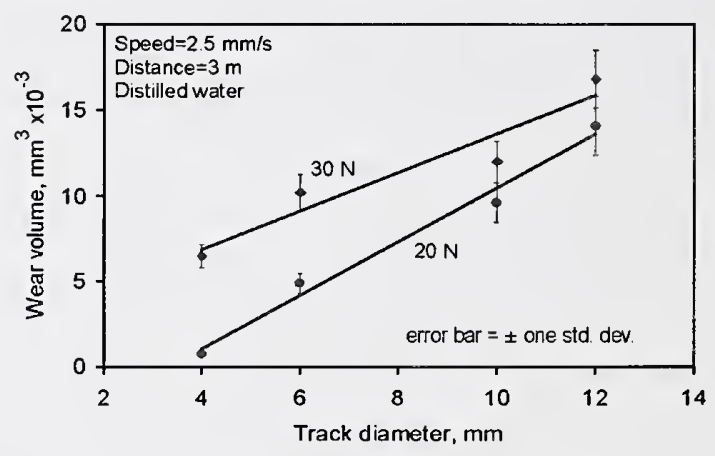

Fig. 2 Wear volume vs. disk track diamete

Test methods and results developed in this project will assist dental materials developers in their search for improved materials, and provide dental practitioners with a sounder basis for selecting and preparing restorative materials. Ultimately the patient will be better served in terms of improved restoration performance and reliability. 


\section{Characteration of Pre- sintered Ceramics}

U.S. industry is pursuing the development of improved processing techniques of advanced ceramics. These improvements require the development and refinement of measurement techniques for bulk density, porosity, moisture, and binder content of pre-sintered ceramics, in both on-line and off-line processing circumstances. Standard reference materials for calibrations of these instruments and evaluated data from round robin tests, such as for bulk density and porosity measurements, provide the necessary foundation for these advances.
Characterization of pre-sintered ceramics directly impacts the cost and quality of the sintered products. Critical information about the pre-sintered green body such as density, porosity, moisture content and distribution predicts the quality of the finished products without actually completing the processing. Consequently, well established characterization techniques are important links in a cost effective manufacturing process.

In this project, parameters and measurement techniques for characterization of porous ceramics are explored, compared, and evaluated. Pre-sintered samples from U.S. ceramic industry and the sensors from equipment manufacturers will be used for the evaluation. The parameters of interest include green density, porosity, moisture content and distribution, binder content and distribution, as well as standard materials for the interlaboratory evaluation and measurement.

A technical report (NISTIR 6543) of assessment in commercially available moisture measurement techniques for ceramic processing was completed. The techniques tested include gravimetric method, near infrared, microwave, proton nuclear magnetic resonance, and magnetic resonance imaging. The strengths and limitations of the techniques were compared. Near infrared can be installed on-line; it is a low cost and simple method; but it has a limited penetration depth and the results are influenced by the color of samples. While microwave techniques allow for deeper penetration depths with advantages of simple operation, low cost, as well as on-line, the high dielectric constant of some materials can complicate the measurement procedure. The emerging NMR and NMR imaging techniques can provide additional information on physical state of water and its spatial distribution in a ceramic sample. These techniques are not widely used due to the high cost, operational complexity and limitation imposed by the size of RFcoil. It is concluded that the gravimetric method should be considered as the primary technique for calibration of all other techniques.

The Materials Science and Engineering Laboratory and the Federal Institute of Materials Research (BAM) of the Federal Republic of Germany have signed an agreement for the joint development, certification (SRM/CRM level), and sales of a calibration material for use with the mercury porosimeter.
This is the first time that an instrumental calibration material will be developed using two different methods of certification that cross national boundaries. The calibration material will be certified to the Standard Reference Material (SRM-NIST) and Certified Reference Material (CRM-BAM) level by both institutions. After certification testing by both institutions, an international round robin testing program will take place with pre-certified laboratories. Statistical analysis of data will be done at NIST and the calibration material will be jointly certified and sold by NIST and BAM.

NIST development and certification testing of SRM 1918 has been completed. A round robin testing program with approximately ten laboratories for the industrial-researchacademic communities is currently under development. Instruments from different manufacturers will be used to check to determine if different intrusion methods will yield differing results for mean pore diameter bulk density and pore size distribution. There is currently no SRM level certified material available to users of this method of analysis. The availability of SRM level materials will enable users to more easily certify their instrumental calibrations with increasingly strict national and international standards for performance and traceability of results.

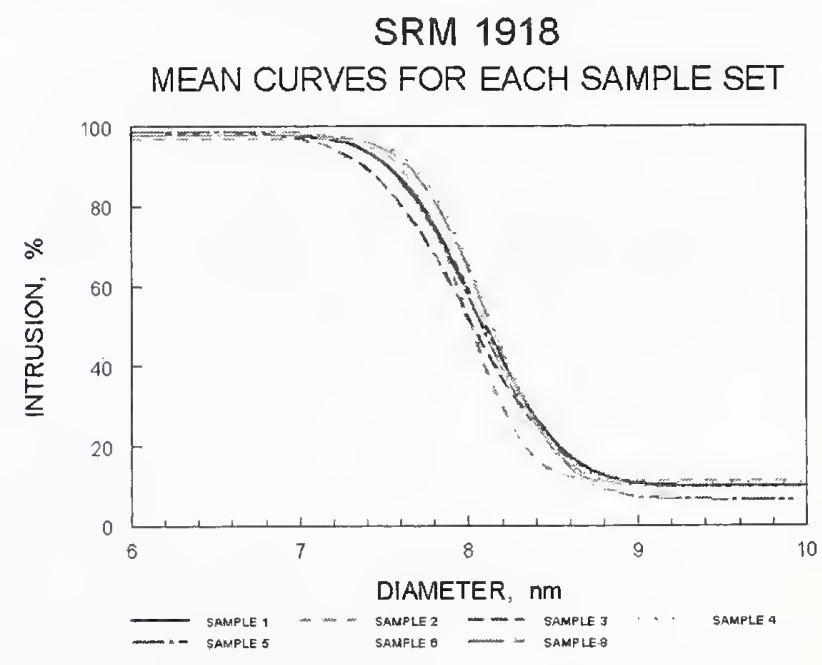




\section{Deformation and Fracture at High Temperature}

This research assists industry in the evaluation, design, and development of advanced structural ceramics for use as hightemperature components in land-based heat engines for power generation and vehicles, and in the development of measurement methodologies for the evaluation of the necessary high-temperature mechanical properties.

\section{Ralph Krause, Jr. and William Luecke}

We are studying the mechanisms and statistics of high temperature creep and rupture in advanced ceramics as well as developing and refining test methods for these measurements.

During 1998 and 1999 we conducted an international round robin for creep rupture of silicon nitride involving 14 laboratories, which comprise a large fraction of the possible laboratories. (Figure 1 shows the results) The analysis of that data is complete and submitted for publication. For $\log _{\mathrm{e}}($ timeto-failure), the within- and between-laboratory coefficients of variation were $15 \%$ and $31 \%$ respectively. The corresponding coefficients of variation for creep rate, which is often the deformation parameter of interest, were $2.5 \%$ and $5.2 \%$, respectively. During 2000 the data and the lessons learned were successfully incorporated into a precision statement for the relevant ASTM standard. The study showed that even reputable laboratories could differ significantly, which was unexpected from previous studies.

We are also characterizing the subcritical crack growth resistance of sapphire, under a program from the Office of Naval Research. Data are needed for lifetime and reliability prediction of windows for missile applications. Preliminary data, the first of its kind, indicate that sapphire has excellent resistance to subcritical crack extension.

We are actively collaborating with industrial as well as academic partners. With Chien-Wei Li of AlliedSignal (now Honeywell) we are characterizing the deformation of a nextgeneration silicon nitride. These data feed into our models for deformation and lifetime. With Michel Barsoum and his student Miladen Radovic we are characterizing the deformation and failure of a new class of machinable, ductile ceramics.
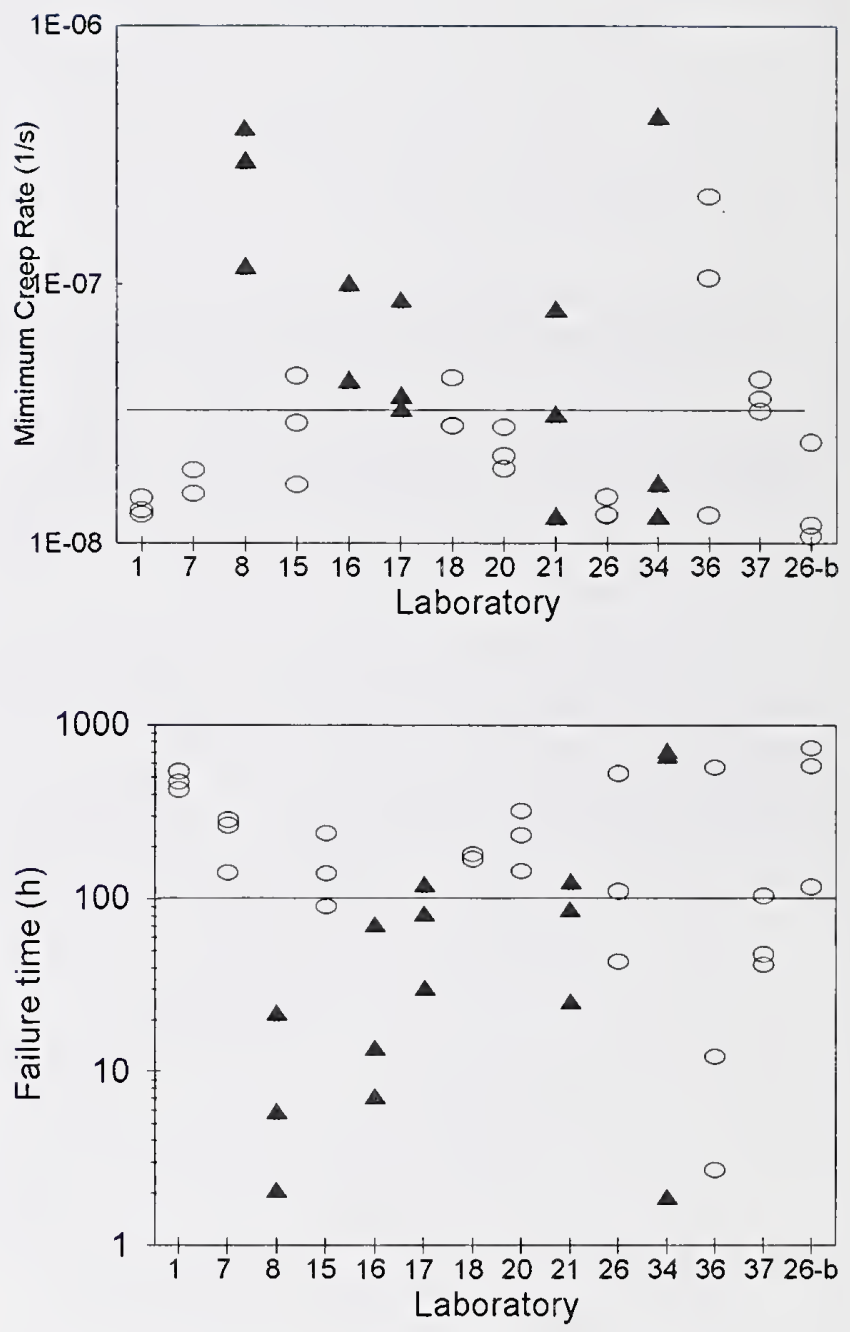

Figure 1. Results of an international round robin on creep rupture of silicon nitride. Solid symbols are from large specimens, open symbols are from small specimens. Solid lines are the grand mean of the data.
Sheldon Wiederhorn (MSEL)

Chien-Wei Li (Honeywell), Frantisek Lofaj (Slovak Academy of Sciences)

Miladen Radovic (Drexel U.) 


\section{Dispersion}

\section{Measurements}

\author{
Vincent A. Hackley
}

The manufacture of advanced ceramics with ultrafine microstructures and enhanced performance requires starting nuaterials that are extremely sniall in scale. To handle such fine particulates, complex mixtures (slurries) are created that include ceramic particles, processing additives and electrolytes in a liquid medium. The distribution of, and chemical interactions between, particulate components and additives in the slurry has a profound influence on the shape forming process. Dispersion measurements play a key role in developing reliable, robust manufacturing processes for advanced materials.

influence of a variety of sample and measurement related factors on the determination of the IEP, including speed of titration, sample preparation, conductivity, aging and particle volume fraction.

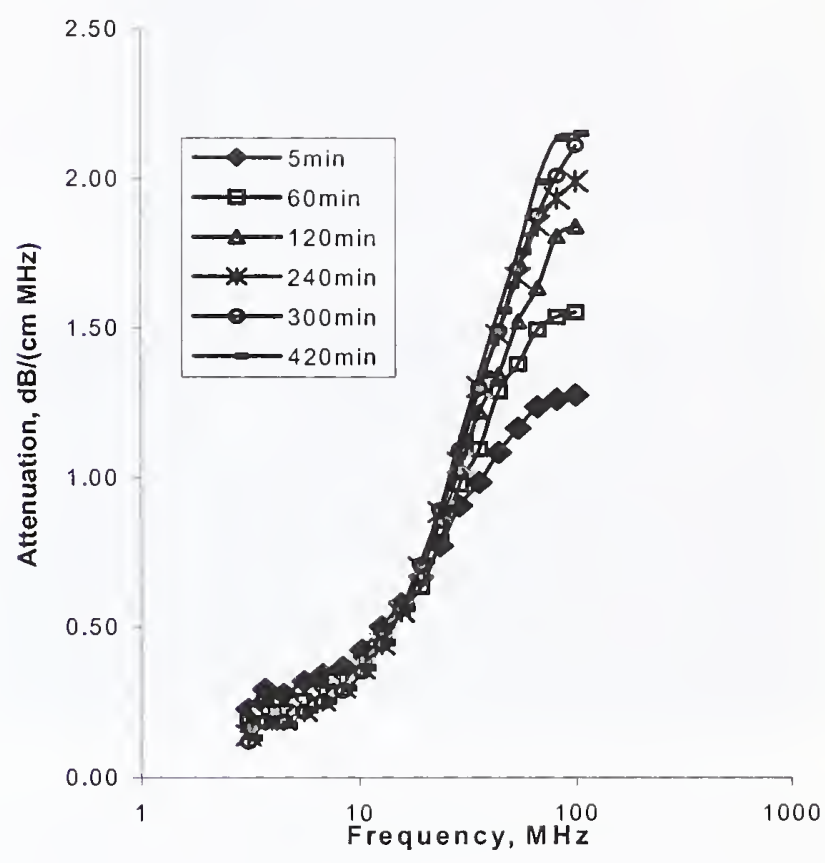

Figure 2. Acoustic attenuation spectra as a function of hydration time for a $2 \%$ volume fraction suspension of portland cement.

We are also developing methods for characterizing industrial suspensions. Such measurements are used to improve reliability and process control, and must cope with high solids concentrations and a complex mixture of components. We are developing methodologies to extract relevant acoustic signatures from amongst substantial noise and under complex and continually varying conditions. This requires that correlations be developed between measured signatures and suspension properties. Figure 2 shows the attenuation spectra as a function of time for a cement suspension undergoing hydration. The shifts in attenuation at specific frequencies is indicative of changes in particle size.

Figure 1. Effect of water quality on electroacoustic mobility and isoelectric point of alpha alumina suspensions.

Vincent Hackley and Lin Lum (Ceramics Division), Chiara Ferraris (Building Materials Division), Ming Tung (Polymers Division), Makio Naito (Japan Fine Ceramics Center), Rolf Waesche (Federal Institute for Materials Research and Testing, Germany), Hemant Pendse (University of Maine), Ungyu Paik

(Hanyang University, S. Korea) 


\section{Effect of Machining}

\section{Damage on Properties of} Ceramics
Manufacturers of ceramic products for diverse applications such as diesel engine fuel injection systems and hip joint replacements require cost effective grinding processes with known or ninimal potential for introduction of performance limiting danlage. This project determines the relationship between machining conditions and performance related requirements such as strength, surface finish, wear resistance, and fatigue life; and provides data, measurement methods to assess the effects of damage, and establishes standard test methods.

\section{Lewis K. Ives and Said Jahanmir}

Grinding with diamond abrasive tools is the predominant machining method for shaping and finishing ceramic components. Because of their brittle nature, ceramics are highly susceptible to the introduction of damage, in particular microfracture, generated during the abrasive/material interaction. This damage can have a critical influence on the capability of finished components to meet performance requirements such as those associated with strength, wear resistance, and fatigue life. An example of microcracks introduce during grinding with a diamond abrasive wheel are shown in the cross-sectioned specimen in Fig. 1. Of particular importance are the large, potentially strengthlimiting cracks (c) extending in from the ground surface.

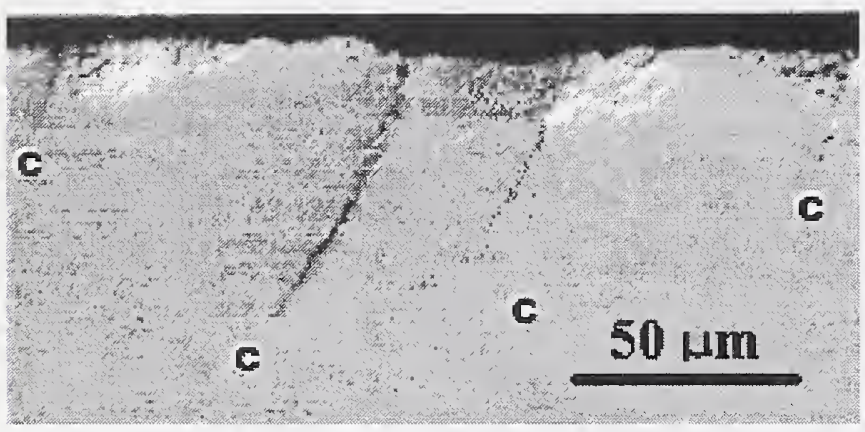

Fig. 1. Cross section through ground silicon nitride surface

In support of manufacturers need for improved grinding processes, this project has focussed primarily on two areas: 1) developing of test methods to measure the extent and effect of grinding damage and 2) determining the influence of grinding conditions on performance related properties of ceramic materials. An example of recent results obtained on an alpha silicon carbide ceramic is shown in Figure 2. The effect on flexure strength of grinding with wheels having two different grit sizes is compared. With a 600 grit wheel, the flexure strength of specimens ground in the transverse and longitudinal direction are about the same, indicating that grinding damage had little or no effect on strength. This was confirmed by fractographic analyses, which showed that fracture was associated with pores and other inherent flaws in the material and not grinding damage. In contrast, specimens ground under the same conditions with a 320 grit wheel in rse direction had a markedly lower strength than specimens

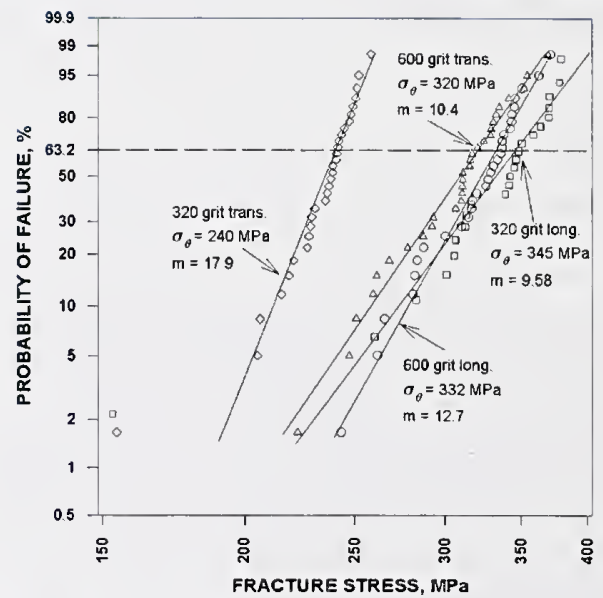

Fig. 2 Effect of grit size on flexure strength of alpha silicon carbide.

ground in the longitudinal direction. Thus, damage introduced by a 320 grit wheel was sufficiently severe to cause a substantial reduction in flexure strength.

In other work, an in-depth evaluation of finish grinding conditions in centerless and cylindrical grinding modes was conducted on a sintered reaction bonded silicon nitride material. Flexure testing of the cylindrical rods was carried out using methods previously developed by this project. This is the first time that such an investigation has been carried out on cylindrical rods and is important because the majority of manufactured parts are prepared by these modes of grinding. In the past evaluation of grinding conditions was done on rectangular specimens prepared with planar grinding methods

Test procedures developed by this project have led directly to the preparation of an ASTM standard test method. The standard is currently undergoing balloting in ASTM Committee C-28 on Advanced Ceramics.

Much of the project work is carried out jointly with members of the NIST Ceramic Machining Consortium. Consortium members are mainly from industry but several universities are also included. The Consortium holds two meetings each year to review ongoing projects and plan future activities. The results generated by the projects are being used by industrial members to optimize their grinding methods and thereby produce more cost effective and reliable products. 


\section{Mechanical Properties of}

\section{Ceramic Membrane}

Materials

\section{Jay Wallace and William Luecke}

This project is divided in two parts. The first part focuses on degradation of candidate ceramic membrane materials during thermal cycling. The second focuses on mechanical properties measurements for next-generation solid oxide fuel cell membranes.

During service, yttria zirconia membranes for solid oxide fuel cells will be thermally cycled as the fuel cell comes on- and off-line. Thermal expansion anisotropy as well as thermal gradient stresses can cause micro- and macrocracking in the membrane. For adequate life-prediction, which is necessary for commercialization, methods to characterize this damage are essential. We have constructed an automated instrumented spherical indenter that can provide local elastic modulus measurements over large areas. Figure 1 shows such a map in a 0.08 mole fraction yttria zirconia (8YSZ) cycled 200 times from room temperature to $900{ }^{\circ} \mathrm{C}$. Clearly there is large variation in the local elastic modulus. We are currently mapping out the time and temperature response. In related work, (Figure 2) we have used constant stress rate testing to demonstrate that $8 \mathrm{YSZ}$ is subject to degradation by subcritical crack growth (SCG). Understanding SCG in this material is central to life-prediction of components.

In a second thrust we have characterized the high-temperature deformation of the prime candidate material to replace $8 \mathrm{YSZ}$ as the fuel cell membrane: Strontium and Magnesium-doped Lanthanum Gallate (LSGM) LSGM is attractive as a solid electrolyte because it operates at much lower temperatures than YSZ. Deformation data indicate that LSGM can possess creep resistance similar to that of $8 \mathrm{YSZ}$. Although the exact mechanism of deformation remains unresolved, evidence points to a diffusional mechanism based on grain boundary sliding.
Solid oxide fuel cells and air separation membranes are expected to perform under severe conditions with lifetimes over $10000 \mathrm{~h}$. To ensure reliable operation, elevated temperature mechanical property data and test methods are required.

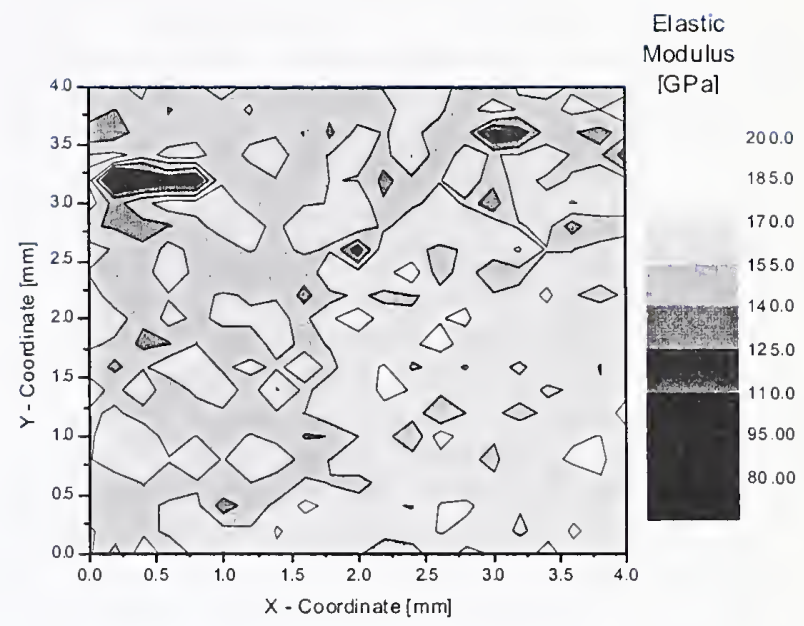

Figure 1. Elastic Modulus variability in $8 \mathrm{YSZ}$ after 200 cycles to $900{ }^{\circ} \mathrm{C}$

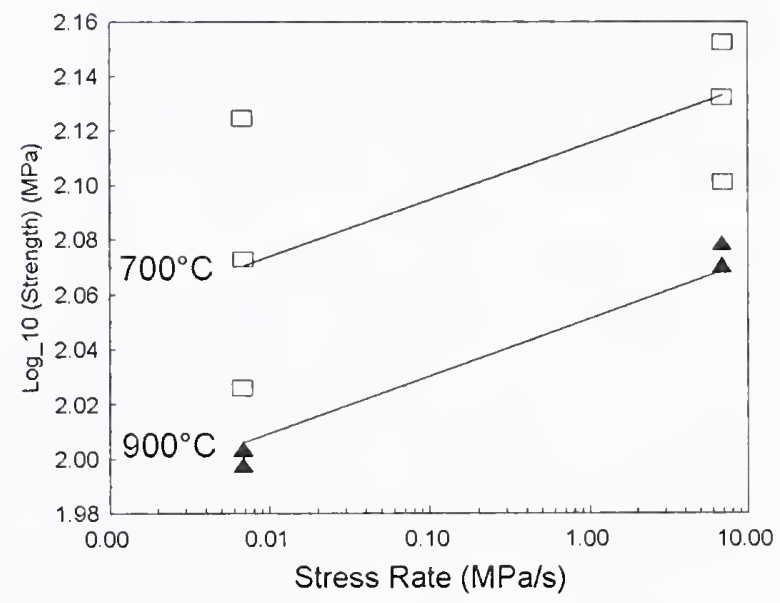

Figure 2. $8 \mathrm{YSZ}$ is subject to subcritical crack growth (SCG) at fuel cell use temperatures, as indicated by the reduction in failure stress at low stressing rates. Data also provide evidence that SCG is thermally activated. 


\section{Mechanical Property Standards}

The goal of this research is to develop standard mechanical test methods and standard reference materials in support of national and international standardization programs. This work encompasses a variety of activities including conducting prestandardization research, performing round robins, writing standards and practices, and creating standard reference materials.

\section{G. Quinn}

The value and impact of this project is growing throughout the advanced ceramics industry. The test methods are sufficiently generic and universal that they may be applied to heat engine, armor, biological, and electronic ceramic materials. For example, a new silicon nitride bearing ball material specification standard, being created in ASTM Committee F-34 with very active European and Japanese participation, utilizes five standards developed in this NIST program. Three ceramic biological material specifications created by ASTM Committee F-04, with very active European participation, include four of the same standards created by this NIST program. Significant cost savings can now be directly attributed to the adoption of several of the standards. Promulgation of a new advanced technique (elastic moduli by impact resonance) has been significantly enhanced by standardization.

This year we completed two ISO standard test methods (room temperature flexural strength and hardness), contributed to the adoption of a third (fracture toughness by single-edged precracked beam), and advanced two others (elevated temperature flexural strength and fracture toughness by the surface crack in flexure method). A major effort on fractographic characterization of strength-limiting flaws in cylindrical rod and rectangular bar flexural strength specimens was completed. This work contributes to four ASTM standards: revision to C 1322, the master fractographic analysis standard; revision to $\mathrm{C} 1161$, the master flexural strength standard, a new standard on evaluating the effects of machining upon ceramic strength; and a new standard test method for flexural strength testing of rods.

We also analyzed and wrote a paper on the effect of chamfers on bend bar resonance for elastic moduli determination in cooperation with Mr. Jeff Swab of the U. S. Army Research Laboratory. The paper will be used as the basis for a revision to 2 ASTM standards that use this method to measure elastic modulus. A series of presentations and publications on the new ASTM and ISO fracture toughness standards as well as the new NIST Standard Reference Material 2100 for fracture toughness were made. SRM 2100 is the world's first reference material for fracture toughness for any class material. We also cooperated with a European round robin on ceramic hardness organized by BAM, Berlin. BAM utilized NIST SRM 2830 Knoop hardness and prototype SRM 2831 Vickers hardness blocks and compared their performance to alternatives. The NIST SRM's were the best in the entire exercise.
Contributors and Collaborators
Collaborations with a variety of USA and International scientists and engineers through ASTM, Versailles Project on Advanced Materials and Standards (VAMAS), International Organization for Standards (ISO), and U.S. Department of Energy programs. 


\section{Powder Size Standards}

\section{James Kelly and Ajit Jillavenkatesa}

We develop and certify glass/ceramic powders as particle size distribution, PSD, Standard Reference Materials (SRMs). These SRMs are needed in industry for instrument calibration, quality control in powder manufacture, and for compliance with ISO 9000 requirements. Necessary to this certification is the development of sampling protocols and size measurement procedures. The primary standards for particle size distribution are a series of glass bead SRMs covering the range from micrometer to millimeter. Maintaining and improving these references is an ongoing effort. Requirements have been identified for several industry specific particle size distribution standards. This year, two reference materials were completed for the thermal spray industry (SRMs 1984 and 1985). These represent the two major WC powder types used by that industry: a fused and crushed powder, and a spray dried and sintered material.

The SRM 1004b (Glass beads with particle size range of $38 \mu \mathrm{m}$ to $125 \mu \mathrm{m}$ ) has been certified for size distribution this year by scanning electron microscopy/computer assisted image analysis. Figure 1 illustrates the PSD for SRM 1004b .

The impact of this work is the availability to industry of a variety of materials and size ranges for particle size standards. There are approximately five hundred (500) units sold each year of the particle size SRMs. Research is underway for the development of glass bead SRMs covering the size range from $1 \mu \mathrm{m}$ to $50 \mu \mathrm{m}$.

A cooperative effort with CENAM has been established with a visiting scientist, Mario Cordero to develop a particle size distribution certified reference material for Mexico.

There is growing interest in the use and application of submicron and nanosized powders. However, the use of such powders is significantly limited by the ability to reliably characterize the particle size distribution of these systems. Efforts have focussed on exploring new techniques and instruments for determination of size distribution. A technique of particular interest that has been studied is based on the settling of particles under the action of centrifugal forces. Various sub-micrometer ceramic particulate systems including silica, alumina, titania, and ceria have been examined by this technique. Influence of techniques of specimen preparation on the observed results have been studied and interpreted. Instrument sensitivity to the state of specimen dispersion, concentration and particle size has been examined. Experience gained through these tests will be utilized in efforts for the development of particle size SRMs in the sub-micrometer range.
This research is directed toward a development of standard reference materials for powder size measurement. Research is extending measurement capabilities to sizes to less than 1 micrometer and a broader range of materials for different applications.
A descriptive text covering important issues encountered during particle size determination has been published as a NIST Recommended Practice Guide. This guide is a compilation of essential facts and some fundamental information about commonly used techniques of particle size analysis in the ceramics industry.

Industrial users have expressed the opinion that such documents give them easy access to varied information about particle size determination in one source. References to national and international standards were of particular interest to all the reviewers. A major raw material producer has expressed interest in providing its national and international customers this practice guide to use as a common basis for comparison of size results in cases of significant discrepancies between the producer and the customer.

NIST testing of SRM 1897 for specific surface area (aluminum silicate) has been completed and a round robin testing program for final certification has begun. Completion of this SRM will conclude a series of new materials available from NIST at the level of SRM certification. The availability of SRM level materials will enable users to more easily certify their instrumental calibrations with increasingly strict national and international standards for performance and traceability of results.

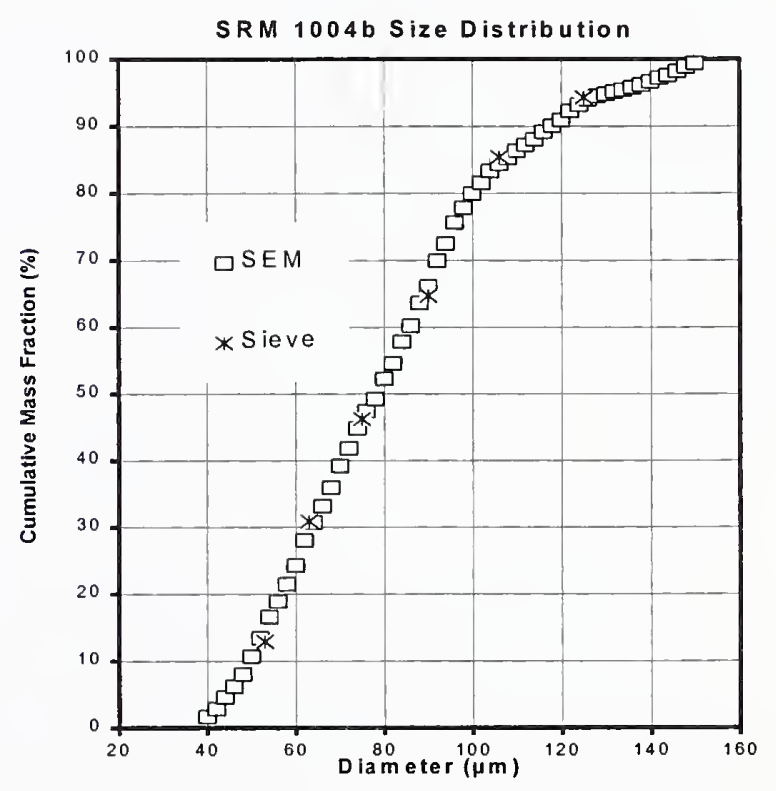

Figure 1. SRM 1004b size distribution
P. Pei, D. Minor (Ceramics Division)

M. Cordero (Mexico), C. Kreller (U.of MD) 



\section{Combinatorial Methods}

The Combinatorial Methods Program develops new measurement techniques and experimental strategies needed for rapid acquisition and analysis of physical and chemical data of materials by industrial and research communities. A multidisciplinary, multi-OU team from the Measurements and Standards Laboratories of NIST participates to address key mission driven objectives in this new field, including needed measurement infrastructure, expanded capability, standards and evaluated data.

Measurement tools and techniques are developed to prepare and characterize materials over a controlled range of physical and chemical properties on a miniaturized scale with high degree of automation and parallelization. Combinatorial approaches are used to validate measurement methods and predictive models when applied to small sample sizes. All aspects of the combinatorial process from sample "library" design and library preparation to high-throughput assay and analysis are integrated through the combinatorial informatics cycle for iterative refinement of measurements. The applicability of combinatorial methods to new materials and research problems is demonstrated to provide scientific credibility for this new R\&D paradigm. One anticipated measure of the success of the program would be more efficient output of traditional NIST products of standard reference materials and evaluated data.

Through a set of cross-NIST collaborations in current research areas, we are working to establish the infrastructure that would serve as a basis for a broader effort in combinatorial research. Within MSEL, novel and elegant methods for combinatorial library preparation of polymer coatings have been designed to encompass variations of diverse physical and chemical properties, such as composition, coating thickness, processing temperature, surface texture and patterning. Vast amounts of data are generated in a few hours that help understand how these variables affect material properties, such as coatings wettability or phase miscibility. Additional focus areas for both organic and inorganic materials include multiphase materials, electronic materials, biomaterials assay, and materials structure and properties characterization. State of the art on-line data analysis tools, process control methodology, and data archival methods are being developed as part of the program.
In order to promote communication and technology transfer with a wide range of industrial partners, an industry-National laboratories-university combinatorial consortium is being organized by MSEL. The consortium will facilitate direct interactions on combinatorial measurement problems of broad industrial interest and efficient transfer of the methods developed to U.S. industry. 


\section{Combinatorial Tools for Dielectric Oxide Thin} Films

\author{
Peter K. Schenck, Debra L. Kaiser
}

Dielectric oxide films are leading candidate materials for next generation wireless, memory and logic devices. Combinatorial methods are ideally suited for the selection of materials for these applications. Industrial participants at the NIST/ARO sponsored Workshop "Combinatorial Materials Science: A National Dialogue" cited a need for measurement tools and databases of information on advanced materials. We are developing novel approaches for library fabrication and high throughput property measurements with the goal of providing a database of processing/property diagrams.
We are engaged in a multi-laboratory project to develop combinatorial tools for the fabrication and characterization of dielectric oxide thin films for wireless communications. These tools will be applied to promising materials for these applications and a database of processing-compositionprocessing information will be generated.

We have designed and fabricated a novel, dual-beam, dualtarget pulsed laser deposition (PLD) system for library film preparation. In the PLD process, the laser beam is split and focused onto two targets of differing composition. The plumes emanating from the two targets (shown below) interact, and a film of continuously variable composition deposits on the substrate. Modifications are planned to introduce additional processing variables (i.e., temperature, laser fluence) into the PLD experiments. In situ imaging and spectroscopic characterization tools in the system will permit real-time control and optimization of the deposition process. The technique is broadly applicable to undoped and doped ceramic, metal and ceramic/metal composite films.

\section{Photograph of plumes from $\mathrm{BaTiO}_{3}$ (yellow) and $\mathrm{SrTiO}_{3}$ (blue) targets in dual-beam PLD system.}

The first system selected for study was $\mathrm{BaTiO}_{3}-\mathrm{SrTiO}_{3}$, a material that is presently being investigated for voltage tunable wireless devices. Library films have been deposited on 50.8 $\mathrm{cm}$ diameter (100)Si wafers at $25^{\circ} \mathrm{C}$ and nominal $10 \mathrm{~mm}$ square $\mathrm{Si}$ substrates at $600^{\circ} \mathrm{C}$ using $\mathrm{BaTiO}_{3}$ and $\mathrm{SrTiO}_{3}$ ceramic targets.
Thickness values, which are required for accurate measurement of the dielectric properties, were mapped by a semi-automated reflectance mode spectrophotometry technique at a spatial resolution of better than $1 \mathrm{~mm}$.

The dielectric properties of $(\mathrm{Ba}, \mathrm{Sr}) \mathrm{TiO}_{3}$ are strongly dependent upon the $\mathrm{Ba} / \mathrm{Sr}$ ratio. Concentrations of $\mathrm{Ba}, \mathrm{Sr}$ and $\mathrm{Ti}$ in a library film were measured by electron microprobe analysis using wavelength dispersive $x$-ray spectrometry (in CSTL). $\mathrm{The} \mathrm{Ba} / \mathrm{Sr}$ ratio, shown in the figure below, varied from 6.5 to 0.5 across the film.

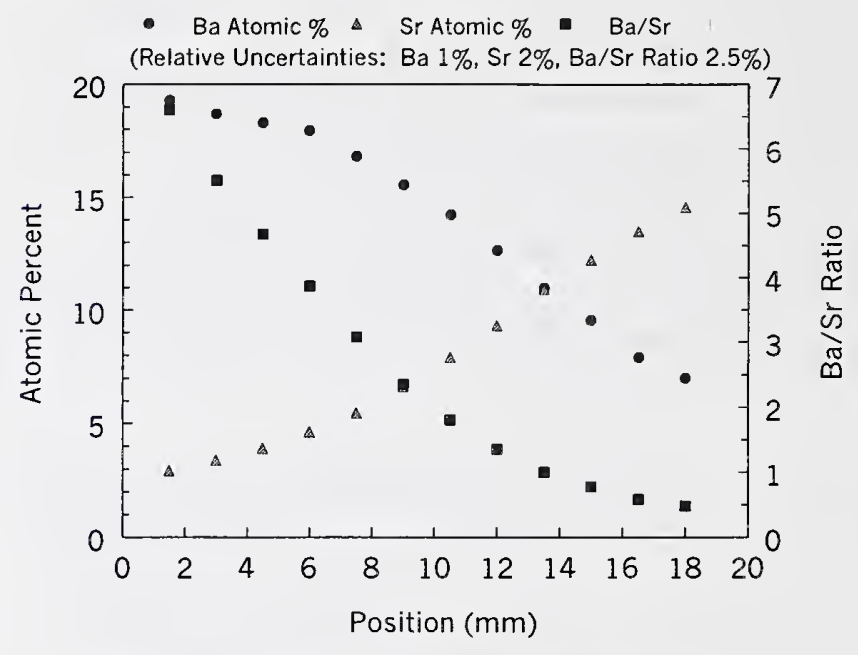

Composition profile of a $\mathrm{BaTiO}_{3}-\mathrm{SrTiO}_{3}$ library.

Our collaborators in CSTL are developing a scanning evanescent microwave microscopy technique for mapping dielectric properties at high frequencies (up to $20 \mathrm{GHz}$ ). Efforts have focused on maximizing the sensitivity and frequency agility of the instrument. Measurements on one of the graded composition $\mathrm{BaTiO}_{3}-\mathrm{SrTiO}_{3}$ film libraries are currently underway.

Combined results on processing conditions, composition and dielectric properties will be stored in a database. Activities are ongoing in ITL to develop informatic protocols and methodologies for handling potentially large amounts of data from this project. 


\section{Data Evaluation and Delivery}

Materials data are critical to the rapid and decentralized design and manufacture of communication, transportation and other devices which characterize 21st century life. The goal of the Data and Data Delivery Program is to provide the producers and users of ceramic materials with the means of fulfilling their data requirements in the most efficient ways. This goal is accomplished by providing improved access to materials data, development of methods for transferring materials data across the WWW, providing protocols for data evaluation, and enhancing the functionality of existing collections of evaluated data. Much of this research is based on information technology and includes the development of a materials mark-up language (MatML), and the linkage of digitized crystallographic information with full structure analysis and phase diagrams produced through the NIST/American Ceramic Society Phase Equilibria Program. Other informatics available to the community is contained in the Ceramic WebBook at the Division Website. The Ceramics WebBook provides links to other sources of ceramic data and manufacturer's information, selected evaluated data sets, structural ceramics and high temperature superconductor databases, glossaries and tools for analysis of ceramic materials. 


\section{Data Evaluation}

Numerous topical studies of the NRC, international workshops of the CODATA, and standards development activities of the ASTM have emphasized the critical role of reliable data in both designing with advanced materials and developing new materials. The issues are two fold, beginning with the need to deduce reliable and consistent property values from diverse, seemingly inconsistent, reported values. Of equal importance is the development of the scientific basis for a general methodology or protocol by which comparable and consistent data evaluations may be pursued independently for other data of special or general interest beyond our own studies.
The issues of data evaluation are being addressed systematically in an approach that recognizes four distinguishable considerations. Each of the four aspects has useful, stand-alone results. When these individual parts are pieced together to comprise a greater whole, the result is the desired, unified methodology. The four stages of data evaluation may be identified as: (I) data collection from selected sources, (II) application of basic evaluation criteria, (III) relational analysis, and (IV) modeling. Stages I and II produce the critical underlying database, while stages III and IV establish the advanced evaluations of the data and the general methodology.

Among the central concerns of data evaluation is the need to understand significant differences among results reported independently for nominally the same properties and materials. Experience has shown that these differences are produced principally by variations in the compositions and mircrostructures of the tested specimens $[1,2]$ or by differences in experimental procedures [3]. By focusing on relatively pure materials, we have found for polycrystalline materials that the principal microstructural effects can be resolved into considerations of grain size and shape, density (porosity or pore size and shape), and the chemical state of grain boundary phases.

In a previous study, for example, we carefully distinguished between the influences of grain size and density on fracture toughness [4] and discovered that the behavior of the critical flaw size was being interpreted incorrectly. Several studies in the literature had reported correlated variations of fracture strength and fracture toughness with the puzzling result that the flaw size was constant. Our analysis however revealed that the flaw size was not constant, but rather that its variation had been masked by concurrent variations in grain size and density.

Pursuing further the influence of microstructure, we are currently examining elastic moduli data for polycrystalline ceramics. This study has the advantage of a well known result, reconfirmed in our analysis, that the elastic moduli are not significantly affected by variations of the grain size. Consequently, we are able to focus fully on the role of density or, more conventionally, the porosity.

Previously studies, dating back more than half a century, had proposed numerous empirical or semiempirical analytical models to describe the porosity dependence. Those efforts ranged from using curves of known shapes to fit the data, to selfconsistent analyses of pores imbedded in an elastic medium. In none of those cases, however, did an analytical expression emerge in closed form from the mathematics of the derivation. Pursuing this goal to obtain a better understanding of the porosity dependence, we applied a theoretical artifice known as an effective medium.

In the effective medium theory [5], we used the classical model of an ionic solid as an idealized reference system in which all the relevant mathematics could be performed. The analysis then imposed a renormalization of the length metric to scale the ideal system to the nonideal system, and the proper retention of porosity, $\phi$, was used as a consistency condition. The result proved to be a simple expression giving the explicit porosity dependence of the bulk modulus in closed form as $B=$ $B_{0}(1-\phi)^{\mathrm{m}}$. We have confirmed that experimental results for a wide range of ceramics indeed are compatible with this model.

[1] R. G. Munro, "Evaluated Material Properties for a Sintered $\alpha-\mathrm{Al}_{2} \mathrm{O}_{3}$, "Journal of the American Ceramic Society, Vol. 80 (8), 1919-1928 (1997).

[2] R. G. Munro, "Material Properties of a Sintered $\alpha$-SiC," Journal of Physical and Chemical Reference Data, Vol. 26, No. 5, pp. 1195-1203 (1997).

[3] R. G. Munro, "Mechanical Properties" in Handbook of Superconductivity edited by C. P. Poole, Jr., Academic Press, pp. 569-624 (1999).

[4] R. G. Munro and S. W. Freiman, "Correlation of Fracture Toughness and Strength," Journal of the American Ceramic Society, Vol. 82, pp. 2246-2248 (1999).

[5] R. G. Munro, "Effective Medium Theory of the Porosity Dependence of Bulk Moduli," Journal of the American Ceramic Society, in press. 


\section{Full Structural}

\section{Crystallographic Data for Non-Organic Materials}

\author{
V. L. Karen and A. Belsky
}

To meet the needs of the industrial, scientific and technical communities, the scope of this project covers three areas: a) building and maintaining state-of-the-art databases containing the structures of non-organic substances, including ceramic, metals and inorganic materials; b) developing software tools for the calculation and standardization of derived data items as well as modules for the intelligent access of these data; and, finally, c) providing access to these databases through modern user interfaces and networking capabilities. Access to crystal structure data can be a key step in solving research and applications problems involving materials, as in the chemical (catalytic materials), petroleum (zeolites), and the electronics (epitaxial growth and thin films) industries. These data are of interest to analysts in areas such as materials design, properties prediction, and compound identification. Better quality data and modern products should help users gain an increased understanding of materials properties and help companies to lower costs and increase research efficiency.

A Cooperative Research Project between NIST and the Fachinformationszentrum Karlsruhe was initiated in June, 1997 to ensure the long term viability and widespread dissemination of the Inorganic Crystal Structure Database, a comprehensive collection of crystal structure data of inorganic compounds containing more than 50,000 entries and covering the literature from 1915 to the present.

In a parallel effort, this year, a collaboration was established with the International Centre for Diffraction Data to fill in missing data in NIST's existing collection of metallic structures. Once completed, this will result in approximately 15,000 new full structural entries abstracted and added to the metals database.

The initial focus of this project has been on been on modernizing and evaluating the Inorganic Crystal Structure Database (ICSD). This has included a complete re-design of the ICSD database structure, conversion and loading of the data into a relational database management system, designing graphical user interfaces to access the data, and creating scientific application modules to analyze the results of a database search. A major effort has been made to design and
The materials community in both science and industry use crystallographic data models on a daily basis to visualize, explain, and predict the behavior of chemicals and materials. Access to reliable information on the structure of crystalline materials helps researchers concentrate experimental work in directions that optimize the discovery process. This project develops, maintains, and disseminates evaluated full structural crystallographic data in modern computerized formats, along with scientific software tools to exploit the content of these databases. implement a Windows-based graphical user interface and underlying search algorithms to create a PC product for the ICSD. This PC prototype is nearing completion. It is tabular in design, allows for searching in five general categories of Chemistry, Crystal Data, Reduced Cell, Symmetry, and Reference Data, and will include enhanced features for the characterization of materials based on lattice and chemistry search modules, and 3-dimensional visualization and powder pattern simulation of inorganic structures.

As entries are added to the ICSD, the data are evaluated by experts in specific disciplines and by specialized computer programs. Several types of evaluation are performed, including examination of an individual data item and looking for consistency within a complete entry. During this year, NIST has initiated a new type of crystallographic evaluation of the ICSD to determine the relationship of an individual entry to the entire database. Scientific strategies and code were developed for the purpose of identifying which entries may represent related and duplicate crystal structure determinations. Database searches have been carried out to locate entries which are identical with respect to reduced cell parameters, space groups, Wyckoff positions, sum formula, among other criteria. Various subsets of the data were prepared and more than 7,000 individual pairs of entries were examined in detail by an expert in crystallography. This expert evaluation will be an ongoing NIST effort.

\section{Contributors S. Young (NIST Standard Reference Data Program) and D. Watson (Cambridge Crystallographic Data Centre, UK) Fachinformationszentrum Karlsruhe, Germany


MatML is an effort to develop a new materials information language and is an application of XML for exchanging materials property data distributed via the World Wide Web.

\section{E.F. Begley}

The materials property data community has expressed the need for an extensible markup language (XML) for exchanging documents containing materials property data on the World Wide Web. NIST is responding to that need by leading the technical effort to develop MatML, a markup language for materials property data. The NIST Systems Integration for Manufacturing Applications Program (SIMA) provided FY00 funding to initiate MatML's development through the establishment of a working group charged with defining the scope and specifications for the new language. The MatML Working Group was established and has not only defined the scope and specifications but also produced a working draft of the new language. Details may be found at $\mathrm{http}: / / \mathrm{www} . c e r a m i c s . n i s t . g o v / \mathrm{matml} / \mathrm{matml} . \mathrm{htm}$.

The MatML Working Group is a cross section of the materials community composed of thirty-eight members from private industry, government laboratories, universities, standards organizations, and professional societies.

NIST participants are from the Ceramics, Polymers, Metallurgy, Semiconductor Electronics, Building Materials, and Materials Reliability Divisions, Center for Theoretical and Computational Materials Science, and Standard Reference Data Program

To learn more about the first six bullets please visit the MatML Web Site at http://www.ceramics.nist.gov/matml/matml.htm.

- Assembly of the MatML Working Group

- Creation of the MatML Discussion Forum

- Design, Development, and Implemention of the MatML Web Site

- Working Group Definition of the Scope and Specifications for MatML

- Development of the MatML Kernel [Note: The MatML Kernel was an early English-language (instead of XML) document outlining the contents of the new language.]
- Development of the Annotated MatML DTD [Note: "DTD" stands for "document type definition" and is the syntactic and semantic formalism that technically describes the new markup language. The Annotated MatML DTD not only contains the working draft of MatML but it also provides English-language descriptions of the components of MatML along with sample code wherein materials property data is "marked up."]

- Publication of the first paper on MatML ["MatML: An XML for Standardizing Web-based Materials Property Data", E.F. Begley and C.P. Sturrock, JOM, July 2000, p. 56]

- Presentation of MatML to visitors as well as NIST staff

\section{Contributors}

ABB ALSTOM Power (UK), Aluminum Assoc., ASM, International, Atomic Weapons Establishment (UK), The Boeing Co., CenTOR Software, Colorado School of Mines, ESM Software, Ford Motor Co., Information Analysis Center, Instron Corp., Iowa State U., Lawrence Berkeley National Laboratory, Massachusetts Institute of Technology, MSC.Software, NRIM (Japan), U., Virtual School of Molecular Sciences (UK), William Andrew Publishing. 


\section{Phase Equilibria Diagrams}

\section{T.A. Vanderah and M.A. Clevinger}

Maintain and develop a state-of-the-art database of critically evaluated ceramic phase equilibria data for industrial and academic customers.

Technical evaluation of phase diagrams culled from the primary literature is carried out by NIST. Preparation of the evaluated diagrams for publication and dissemination is carried out at NIST by personnel of the American Ceramic Society (ACerS) supported by funds raised by the Society from industry, academia, and individuals. This collaboration of more than 60 years represents an agreement with ACerS to provide evaluated phase diagrams for the ceramic industry. The phase diagrams are supplied either in printed form or in computerized versions, and are distributed through the ACerS.

The current database was created in the mid-1980's and is currently undergoing a complete modernization. The HPbased system containing approximately 35,000 text and graphics entries will be integrated into a modern, relational database hosted on a PC-based platform. The new system will be capable of electronic publishing in a variety of formats, including a Web-based version. Much of this year's efforts have been assisting and working with the software vendor to design and build the new system, which must incorporate all of the scientific data relationships embodied in the original database. In addition, NIST-ACerS data center personnel have substantially completed required modernization tasks including upgrading of the digitization software originally written by NIST staff, and input of 2,000 commentaries and 6,955 diagrams from older volumes of the series that did not exist as electronic files.

Electronic input of Volume XIII of the series was also completed. This volume, edited by R.S. Roth, contains phase diagrams pertinent to oxide systems. Publication of this volume will occur upon completion of the modernization process in 2001. A second monograph entitled "Oxides of $\mathrm{Ti}, \mathrm{Nb}$, and Ta: Electronic Ceramics I", is now in progress, and will be edited by R.S. Roth and T.A. Vanderah. Most of the systems to be included in this volume will be of major interest to the fields of dielectric, ferroelectric, and piezoelectric ceramics.

\section{Contributors and Collaborators}





\section{Magnetic Materials}

Magnetic materials are pervasive throughout our society. They are used in magnetic recording media and devices, in all motors and transformers, on credit cards, in numerous types of magnetic sensors, in magnetic resonance imaging (MRI) machines, in microwave communications, in magnetic separation, and in magnetic cooling. Magnetic materials include metals, ceramics and polymers at different size scales ranging from large castings to particulates, thin films, multilayers and nanocomposites.

In the present trend to make devices smaller, new magnetic materials are constantly being developed. One critical need for implementation of these materials is the development of the measurement science needed for their characterization. This is the focus of the Magnetic Materials Program. Proper measurements of key magnetic properties, determination of the fundamental science behind the magnetic behavior of these new materials, analyses of the durability and performance of magnetic devices and development of Standard Reference Materials are key elements of this program. Some information is only obtainable by the use of unique measurement tools at NIST like the neutron diffraction facilities at NCNR, or the magneto-optic indicator film apparatus for observation of magnetic domain motion. Of particular interest is understanding the magnetic behavior of low dimensional systems, in which one or more characteristic dimensions have been reduced to nanometer sizes.

Areas of present study include preparation, characterization, and modeling of multilayers and other low-dimensional systems for optimized giant magnetoresistance effect and magnetocaloric effect, and spintronic systems wherein spin dependent magnetic devices are integrated directly into semiconductor chips. Giant magnetostriction alloys, prepared using combinatorial methods, are similarly analyzed. Observation and micromagnetic modeling of magnetic domains play a key role in understanding magnetization statics and dynamics. Advanced magnetic measurements are developed for a wide range of materials including weld metal ferrite standards, and are applied to a wide range of magnetic phenomena including magnetic exchange bias, magnetic susceptibility of small samples at high frequencies, and magnetization time response to a change in magnetic field. Magnetic measurement standards are prepared and certified.

Nanotribology of magnetic hard disks addresses the issue of durability and performance of the magnetic storage technology. It provides measurement techniques on friction, stiction, and ways to lubricate the hard disks to enable higher and higher areal densities.
By experimentally addressing important issues in magnetism, by bringing together the industrial and scientific communities through the organization of workshops and conferences in the area, and by the development and preparation of appropriate standards, NIST acts to accelerate the utilization of advanced magnetic materials by the industrial sector, and to enable industry to take advantage of new discoveries and innovations. In addition, close linkage with the national storage industry consortium (NSIC) which consists of 38 companies and a score of universities allows industrial relevance and partnership. Additional collaborations with Xerox, General Motors, Hewlett Packard, IBM, Seagate, and Motorola Corporations, for example, enable NIST to leverage its activities with the much larger, but complementary, capabilities of other organizations. 


\section{Nanotribology}

\section{Stephen Hsu, Richard Gates, Patricia McGuiggan}

The effectiveness of the lubricant and the diamond-like carbon overcoat to protect the magnetic hard disks from wear is key to higher data storage density and data transfer rate. Working with the National Storage Industry Consortium Tribology Working Group, we address the measurement needs of friction, stiction, and wear and means of controlling wear at the nanometer scale. We also explore the fundamental concept of designing and organizing novel molecular assemblies to achieve better wear protection for the disks in conjunction with our CRADA partner Pennzoil-Quaker State Company.

\section{Head disk interface study}

Current magnetic hard disks are protected by one nanometer thick perfluoropolyethers (PFPE) and $7.5 \mathrm{~nm}$ thick carbon overcoat. In order to increase the areal density, the magnetic spacing (the distance of the sensor to the middle of the magnetic layer) needs to be decreased from $30 \mathrm{~nm}$, the current distance, to $10 \mathrm{~nm}$. This results in a flight height of about $5 \mathrm{~nm}$. At this flight height, occasional collisions between the head and disk may be unavoidable since manufacturing technology cannot produce such flatness and smoothness. In addition, with the increase of areal density, it is desirable to increase the data transfer rate to take advantage of the sensor capability. Higher data transfer rate means the linear velocity of the head will be increased from the current $10 \mathrm{~m} / \mathrm{s}$ towards $40 \mathrm{~m} / \mathrm{s}$. As the speed is increased, potential high speed impacts due to the waviness of the disk, rotation wobbles, and roughness of the surface increase significantly. We have initiated an effort to develop a onepass high-speed impact test to quantify the resulting damage.

\section{Monolayer film design and organization}

With the decrease in flight height and increase in speed, the need to protect the integrity of the magnetic layer becomes more critical. The decrease of the diamond-like carbon overcoat thickness to allow higher areal density makes the effectiveness of the lubricant layer critical.

Current lubricant Z-Dol (a PFPE with two alcohol functional groups) works by diffusing into the contact area after the head passes over, thus creating a "self-repairing" property. However, the lack of bonding with the surface makes it relatively weak in resisting high shear impacts. We have proposed to organize a monolayer molecular assembly with different molecular species. Some species will bond strongly with the surface providing high shear resistance, some species will be mobile to provide "self-repairability", and some species will cross-link under certain temperature and shear conditions (typical of the contact conditions under high speed sliding contacts). Such a molecular assembly, if successful, will provide a much higher degree of protection of the magnetic sub-layer. There are potential applications of such a concept to microelectromechanical (MEM) and other

\section{microsystems.}

Research on Self-Assembled Monolayers (SAM) suggested poor durability in a hard disk system. Subsequent research suggested that a minimum molecular weight of 1,000 is needed to control friction and wear. Different customsynthesized molecules with various functional groups and different molecular weights are required to validate this theory. Thus, we signed a CRADA with Pennzoil-Quaker Co. to test the validity and viability of implementing such molecular assemblies for wear control.

Using dip-coating (single and successive dipping) and vapor phase deposition (single and successive coating), we were able to deposit 3 to 4 components onto a hard disk surface with an overall thickness of about one nanometer. Durability test results suggested much improved resistance to shear.

\section{Monolayer film characterization}

While we can successfully deposit 3 or 4 molecules on an engineering surface such as a magnetic hard disk, the spatial distribution of different species and their stacking characteristics are of profound significance. Ultra-soft X-ray near edge fine structure spectroscopy at the Brookhaven Synchrotron Radiation Facility has been used and has been successful in determining the relative concentration of each species and the nature of interactions between the monolayers with a disk surface. Collaboration with Wayne State University on high resolution modulated AFM provides additional insight of where the molecules are on a typical surface.

We are now initiating an effort to study the chemical nature of the diamond-like carbon overcoats using Nuclear Magnetic Resonance (NMR) and Electron Spin Resonance (ESR). Disks of different chemistries $(\mathrm{CHx}, \mathrm{CNx}$, and $\mathrm{ChxNy}$ ) from the National Storage Industry Consortium (NSIC) will be characterized in terms of the surface energy, bonding characteristics, and interaction pattern with monolayers.
Contributors and Collaborators
Participants: Dan Fischer (Ceramics), Frank Yin (UMD/NIST), Li Na (NIST), Sujeet Singha (NIST), Singh Batia (IBM), Jun Zhang (DSI), G. Y. Liu (Wayne State), Frank Talke (UCSD), Selda Gunsel (Pennzoil), J. Gui (Seagate), Aric Menon (Readrite), Ben Dekoven (Intevac/MMC), G. Walker (Quantum), M. Dugger (Sandia), J. Luo (Analog Devices), P. Mazumdar (NIST/ATP) 
Today's U.S. microelectronics and supporting infrastructure industries are in fierce international competition to design and produce new smaller, lighter, faster, more functional, and more reliable electronics products more quickly and economically than ever before.

Recognizing this trend, in 1994 the NIST Materials Science and Engineering Laboratory (MSEL) began working very closely with the U.S. semiconductor, component and packaging, and assembly industries. These early efforts led to the development of an interdivisional MSEL program committed to addressing industry's most pressing materials measurement and standards issues central to the development and utilization of advanced materials and material processes within new product technologies, as outlined within leading industry roadmaps ${ }^{1}$. The vision that accompanies this program - to be the key resource within the Federal Government for materials metrology development for commercial microelectronics manufacturing - may be realized through the following objectives:

- Develop and deliver standard measurements and data;

- Develop and apply in situ measurements on materials and material assemblies having micrometer- and submicrometer-scale dimensions;

- Quantify and document the divergence of material properties from their bulk values as dimensions are reduced and interfaces contribute strongly to properties;

- Develop fundamental understanding of materials needed in future microelectronics.

With these objectives in mind, the program presently consists of twenty separate projects that examine and inform industry on key materials-related issues, such as: electrical, thermal, microstructural, and mechanical characteristics of polymer, ceramic, and metal thin films; solders, solderability and solder joint design ${ }^{2}$; interfaces, adhesion and structural behavior; electrodeposition, electromigration and stress voiding; and the characterization of next generation interlevel and gate dielectrics. These projects are conducted in concert with partners from industrial consortia, individual companies, academia, and other government agencies. The program is strongly coupled with other microelectronics programs within government and industry, including the National Semiconductor Metrology Program (NSMP) ${ }^{3}$ at NIST.
The NSMP is a national resource responsible for the development and dissemination of new semiconductor measurement technology.

More information about this program, and other NIST activities in Materials for Microelectronics can be found at: http://www.msel.nist.gov/research.html

${ }^{1}$ International Technology Roadmap for Semiconductors, 1999, and National Technology Roadmap for Semiconductors, 1994 and 1997, Semiconductor Industry Association, San Jose, CA; National Technology Roadmap for Electronic Interconnections, IPC, Lincolnwood, IL, 1995, 1997; National Electronics Manufacturing Technology Roadmap, National Electronics Manufacturing Initiative, Inc., Herndon, VA, 1996, 1998, 2000.

${ }^{2}$ http://www.ctcms.nist.gov/programs/solder ${ }^{3} \mathrm{http}: / /$ www.eeel.nist.gov/810.01/index.html 


\section{Deposition and}

\section{Properties of Ultrathin Dielectric Oxide Films}

\author{
Debra L. Kaiser, Igor Levin
}

The International Technology Roadmap for Semiconductors projects that traditional silicon dioxide gate stacks will need to be replaced by higher $\kappa$ dielectric materials by the year 2005 to meet the industry's device scaling goals. As a result there has been intensive research on alternative gate dielectric materials. We have developed spin coating processes for the deposition of ultra-thin $(<5 \mathrm{~nm})$ films of $\mathrm{ZrO}_{2}$ and $(\mathrm{Ba}, \mathrm{Sr}) \mathrm{TiO}_{3}$, two alternative high $\kappa$ materials. The films are being used to develop nano-scale compositional metrology tools and to investigate processing/structure/property relationships.
It is projected that oxide equivalent thicknesses below $1.5 \mathrm{~nm}$ will be required for the gate dielectric layer in complementary metal oxide semiconductors transistors within the next 5 years ${ }^{1}$. Predictions of unacceptably high tunneling currents in sub-1.5 $\mathrm{nm} \mathrm{SiO} \mathrm{S}_{2}$ dielectric layers have spurred research into alternative higher dielectric constant gate materials. Candidate materials under consideration are unary oxides such as $\mathrm{Ta}_{2} \mathrm{O}_{5}$, $\mathrm{TiO}_{2}, \mathrm{ZrO}_{2}$, and $\mathrm{HfO}_{2}$, complex oxides such as $(\mathrm{Ba}, \mathrm{Sr}) \mathrm{TiO}_{3}$ and $\mathrm{LaAlO}_{3}$, and silicates. Characterization of ultra-thin layers of these materials requires the development and application of electrical, structural and compositional metrology tools.

In collaboration with the CSTL, we initiated a project in FY99 on compositional metrology for alternative gate stack materials. Initial efforts focused on developing capabilities to analyze the composition of a model material, chosen to be $(\mathrm{Ba}, \mathrm{Sr}) \mathrm{TiO}_{3}$ (BST), with film thickness ranging from hundreds of nm (where metrology tools exist) down to a few nanometers. A spin coating process was developed to deposit BST films of precisely known composition from a highly stoichiometric $[(\mathrm{Ba}+\mathrm{Sr}) / \mathrm{Ti}=1.00 \pm 0.01]$ liquid precursor solution onto 50.8 $\mathrm{mm}(100)$ Si wafers. By optimizing the solution composition and spinning conditions, films with thicknesses down to $2 \mathrm{~nm}$ as measured by $\mathrm{x}$-ray reflectometry and high resolution electron microscopy (HREM) have been fabricated. BST films deposited directly on silicon have an $\mathrm{SiO}_{x}$ interfacial layer, as seen below in the image of one of our films. Analytical methods and correction procedures have been developed to measure the composition of BST films by electron probe microanalysis.

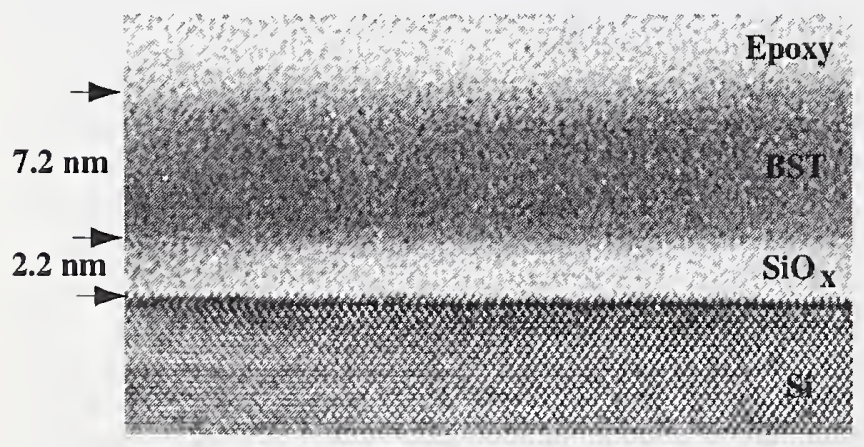

HREM image of a BST thin film $(\approx 7 \mathrm{~nm})$
Subsequent work by groups outside NIST indicated that BST is not a viable gate dielectric material because it is unstable on $\mathrm{Si}$ and has unacceptable electrical properties. Our efforts in FY2000 have shifted to $\mathrm{ZrO}_{2}$, a promising unary oxide gate dielectric for the $100 \mathrm{~nm}$ and $70 \mathrm{~nm}$ technology nodes. We have developed a process for spin coating $\mathrm{ZrO}_{2}$ films onto (100) Si wafers from solutions containing zirconium acetate, propylene glycol, isopropanol and glacial acetic acid. The coated wafers are dried at nominal temperatures of $80^{\circ} \mathrm{C}, 200{ }^{\circ} \mathrm{C}, 450{ }^{\circ} \mathrm{C}$ and $580^{\circ} \mathrm{C}$ in air. By varying the processing conditions (solution composition, ramp rate and spin rate), $\mathrm{ZrO}_{2}$ films with thicknesses ranging from about $40 \mathrm{~nm}$ to $2 \mathrm{~nm}$ have been fabricated. Although the silicon wafers are subjected to an HF strip before coating to remove the native oxide, an amorphous $\mathrm{SiO}_{\mathrm{x}}$ layer is formed between the film and substrate as illustrated in the HREM image below. The $\mathrm{ZrO}_{2}$ layer is crystalline with a preferred $<111>$ orientation. The films appear to be smooth and have uniform morphology.

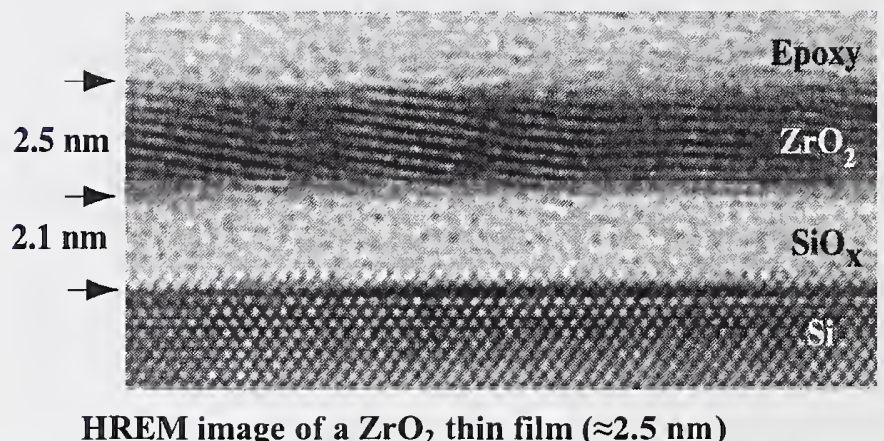

Electrical contacts of $\mathrm{Cr}(5 \mathrm{~nm}) / \mathrm{Au}(100 \mathrm{~nm})$ have been deposited onto BST and $\mathrm{ZrO}_{2}$ spin-coated films. Capacitance and leakage current measurements are in progress in EEEL. Planned work includes exploring alternative processing approaches to reduce the $\mathrm{SiO}_{\mathrm{x}}$ layer thickness and determining the effect of structure (amorphous vs. crystalline) on the electrical properties of $\mathrm{ZrO}_{2}$ films.

${ }^{1}$ International Technology Roadmap for Semiconductors, 1999 Edition, p. 110.
Contributors

and

Collaborators
Chuck Bouldin and Joe Ritter, Ceramics Division

Ryna B. Marinenko, John T. Armstrong, Eric Steel, Chemical Science and Technology Laboratory (CSTL)

Curt A. Richter, Electronics and Electrical Engineering Laboratory (EEEL) 


\section{Domain Stability in Ferroelectric Thin Films}

\author{
Grady S. White, John E. Blendell
}

Ferroelectric thin films have the potential for making major inipacts in applications as diverse as non-volatile RAM, uncooled detectors, and MEMS. The material properties required for these applications, switchable electric dipoles, large pyroelectric coefficients, and piezoelectric response, are all controlled by the domain stability in the films. The relationship between microstructure and the physics of domain stability has never been established. We are developing measurement techniques which allow real time determination of the domain behavior.
We are measuring domain stability in $\mathrm{Pb}(\mathrm{Zr}, \mathrm{Ti}) \mathrm{O}_{3}(\mathrm{PZT})$ thin films as a function of film microstructure and electric history. Although domain stability issues, i.e. pinning, aging, fatigue and retention, limit the use of ferroelectric films in nonvolatile memory and micro-actuator applications, the physics and materials science that affect them are not understood. We have demonstrated that domain pinning in a PZT film appears to be associated with specific grain boundary sites; different sites pin domains either up or down. These sites can occur at different locations along the grain boundary. We are currently examining these sites to ascertain what features of the grain boundary control pinning.

The technique used to monitor domain motion is based on the piezoelectric response of a film to an applied electric field (J. Vac. Sci. Tech. B, 14[2], 602 (1996)). In the measurements, an applied AC electric field $(\approx 1 \mathrm{MV} / \mathrm{m})$ changes the thickness of the ferroelectric film due to piezoelectric response. Such changes are observed by atomic force microscopy (AFM) and the phase shift of the response is related to the orientation of the polarization of the sample. The spatial resolution of the technique is limited to $\approx 20 \mathrm{~nm}$ due to the contact area of the tip and spreading of the electric field. The response varies from in-phase response (polarization in the direction of the applied field) to response which is $180^{\circ}$ out of phase (polarization in the opposite direction to the applied field). Figure 1 illustrates the measurement method.

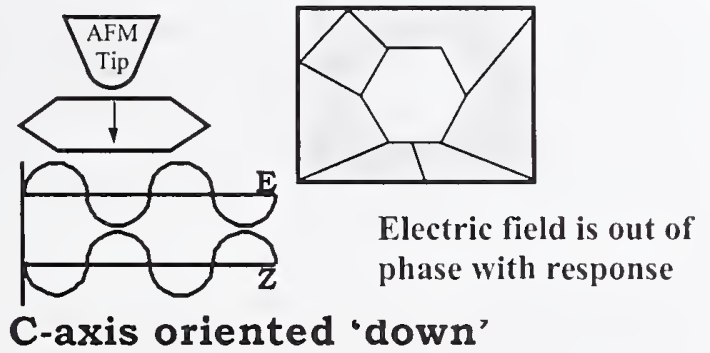

Figure 1. Schematic of AFM domain imaging. A conductive AFM tip is scanned over the film and the center region (bright) indicates that the domain orientation is opposite that of the surrounding regions (dark). Designations of "down" or "up" represent $180^{\circ}$ phase changes and are not aligned with the laboratory coordinate system.
As shown below, grain boundaries are the strongest pinning sites, with the adjacent regions resisting $180^{\circ}$ polarization changes. The grain boundary regions also initiate the rapid relaxation of the domains back to their original orientation. Modeling of the stresses which may drive this reversal are being carried out using an object oriented finite element technique (OOF) developed at NIST.

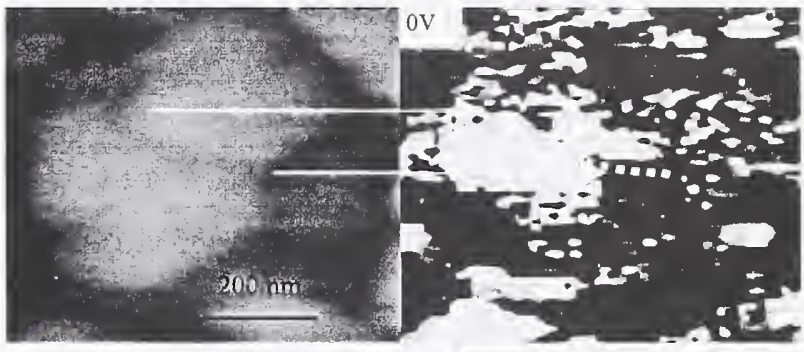

Figure 2: Top left image is AFM topograph of a portion of a PZT thin film. 1mages in the right column show the phase response of the film to $1 \mathrm{~V}_{\mathrm{rms}}$ driving voltage as a function of a applied bias $\mathrm{V}_{\mathrm{DC}}$. From top to bottom, $\mathrm{V}_{\mathrm{DC}}=0 \mathrm{~V},+1 \mathrm{~V},+2 \mathrm{~V}$, and $0 \mathrm{~V}$. The dotted line indicates the location of the grain boundary observed in the topograph image. The out-of-phase response (bright region) around the grain boundary shrinks as $V_{D C}$ increases but reemerges when $V_{D C}$ is removed. Note that the domain behavior is not uniform along the length of the grain boundary.
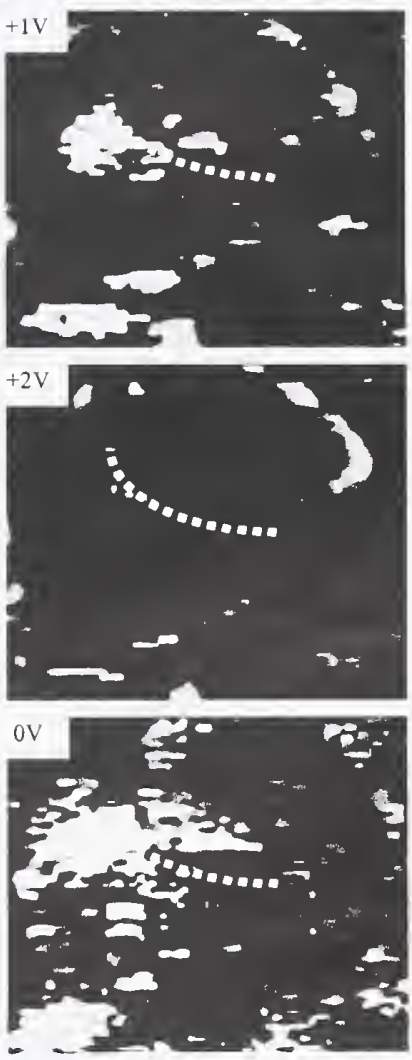


\section{Texture Measurements in} Thin Film and Bulk

\section{Materials}

\author{
Mark D. Vaudin
}

Many thin film and bulk materials used in electronic applications have a preferred crystallographic orientation or texture. Properties of materials can be strongly affected by texture. To optimize the development and application of textured materials, it is desirable to quantify the effects of texture on properties, which requires accurate characterization tools. NIST has developed quantitative texture measurement techniques that employ equipment commonly available in most industrial and academic settings.
The properties and performance of electronic devices can be strongly dependent upon the texture of the various material layers. For example, the remanent polarization in $\mathrm{PbZr}_{x} \mathrm{Ti}_{1-\mathrm{x}} \mathrm{O}_{3}$ (PZT) films used in nonvolatile RAM is orientation-dependent, so the ability to switch domains during a writing operation is strongly influenced by the PZT texture. Quantifying texture effects on properties requires accurate measurement of the texture. This project addresses issues relevant to texture measurement with particular emphasis on electronic materials.

Classical texture measurement methods require specialized equipment that is not routinely available in most laboratories. We have developed an accurate technique for measuring fiber (i.e., axisymetric) texture that uses a conventional $\theta-2 \theta \times$-ray diffractometer. In our approach, $\theta-2 \theta$ and $\omega$ scans are collected from the specimen and corrected for defocusing and absorption effects using custom software developed for this project. Accurate texture profiles are calculated from the corrected data. Volume fractions of various textured populations can be determined for films with multimodal texture from the texture profiles and the $h \mathrm{kl}$ Bragg intensities relative to intensities from untextured films of the same phase. For example, the figure below shows texture profiles for a $\mathrm{Ba}_{\mathrm{x}} \mathrm{Sr}_{1-\mathrm{x}} \mathrm{TiO}_{3}$ (BST) film with mixed (100) and (110) texture. The volume fractions of $(100)$ - oriented $(60 \% \pm 5 \%)$ and (110)-oriented $(40 \% \pm 5 \%)$ material

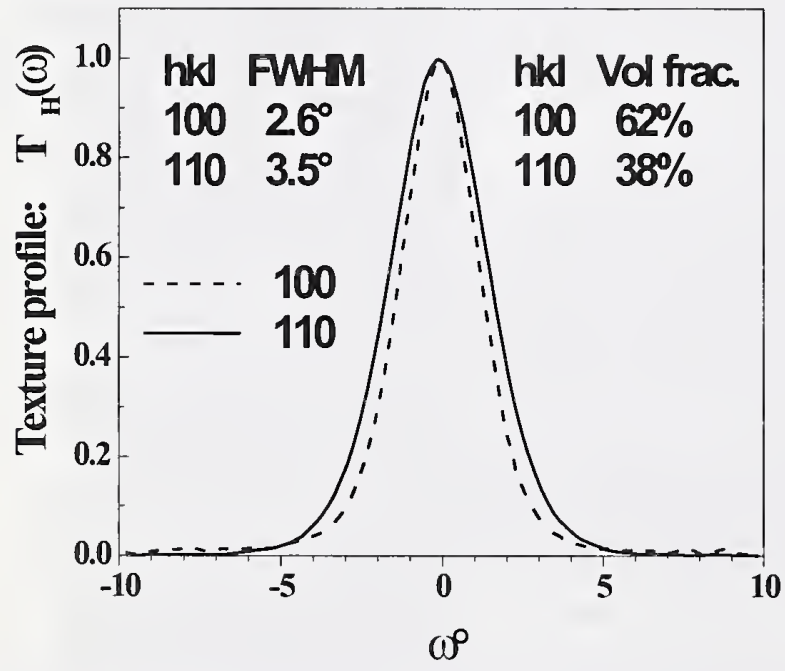

calculated using our more comprehensive method are significantly different from the values of $75 \% \pm 5 \%(100)$ and $25 \% \pm 5 \%(110)$ based only on integrated peak intensity measurements.

Our TexturePlus software package is available on the web at http://www.ceramics.nist.gov/webbook/TexturePlus/texture.htm. Tools for simple analysis of $\theta-2 \theta$ patterns and integration of corrected texture profiles for volume fraction determinations are included. IBM and Ramtron Corp. have used our technique to measure texture in BST DRAM films and PZT nonvolatile RAM films respectively.

We organized and held a workshop on Texture in Electronic Applications at the NIST Gaithersburg site on October 10 and 11, 2000 . The primary goal of the workshop was to provide a forum for the discussion of critical issues relevant to texture and texture measurement. The major topics were:

- Production and control of texture in a variety of different device materials

- Different methods of texture measurement and conditions for which each method is applicable

- Texture analysis procedures

- Effects of texture on properties and performance

- Effects of texture on processing of subsequent layers deposited upon a textured template

A deliberate effort was made to engage participants involved in a broad array of materials, measurement techniques and application areas in order to provide an opportunity for meaningful interchange and collective insight into the measurement needs of the texture community. The nearly 40 attendees were evenly divided between industry, universities and national labs. During roundtable discussions, it became clear that there is a strong need for texture standards. A prerequisite for standards development is interlaboratory comparisons of results obtained on the same specimens with different techniques, and also on the same specimens using the same technique but different equipment. NIST agreed to take the lead in organizing such an activity, and will continue to design and validate texture measurement procedures for the specific needs of the texture community. A report on the Workshop will be published in the NIST Journal of Research. 


\section{Materials for Wireless Communications}

The current revolution in wireless communications would not have been possible without the discovery and development of oxide ceramics exhibiting the coincidence of high, temperature-independent permittivity with low dielectric loss. Advanced ceramics are primarily used as building blocks for filters and oscillators in components that are critical to the performance of the end application. One of the first ceramics widely used for cellular base stations was discovered, and its processing phase diagram determined, in a collaborative effort between Bell Labs and NBS in the early 1970's. Today, wireless technologies constitute one of the most important growth areas in the world electronics industry. Paramount in the costcutting and miniaturization process is the need for research that will facilitate the rational design of advanced materials to provide temperature stability, frequency, and size-reduction requirements for the next generation of devices for cellular, PCS, and many other niches of the wireless communications industry.

Technically important ceramic materials fall into two major dielectric categories; namely, bulk ceramics for base station resonators/filters, and those needed for low-power, miniaturized hand-held devices requiring thick and/or thin films in an integrated structure. Ceramics Division activities include research on both types of dielectric materials. The primary goal of the program is to determine methods that can be used to predict high-frequency dielectric behavior to enable rational design of next-generation ceramics.

Current research on ceramic materials for base station applications includes experimental determination of selected complexoxide phase diagrams integrated with in-depth structural (Xrays, electrons, neutrons), crystal-chemical, spectroscopic, and dielectric property characterization. The objective of this multidisciplinary project is to determine the fundamental relationships between phase chemistry, crystal structure, and dielectric performance at wireless frequencies. This work is performed in collaboration with personnel in the Radio-Frequency Technology Divisions.

Another project uses first-principles (FP) methods to elucidate the roles of cation order-disorder and ferroelastic phenomena in determining the phase relations and physical properties of complex ceramic oxide systems. Typically, these materials exhibit exploitable ferroelectric, dielectric, or magnetic properties, and are widely used in technical applications such as transducers, multilayer capacitors, dielectric resonators, or dielectric filters. First- principles calculations are used to predict cation ordering phenomena, physical properties, and how they vary with chemical composition. Critical experiments are performed to test the predictions. An additional technical objective is to benchmark various FP techniques that are used to compute physical properties and the formation energies on which the calculations are based.

Microstructural modeling and experimental studies are also underway to determine the dimensional changes in lowtemperature-cofired ceramics used for portable communication devices. Models developed in this study were identified by a large segment of industrial producers as being crucial toreducing the time for the design and production of components.

Related work in the Materials Reliability Division includes development of noncontact acoustic metrology to characterize wireless materials in both thin-film and bulk form. The mechanical properties of thin films are investigated using laser-ultrasonic methods to generate and detect surface acoustic waves. The elastic-property information obtained will result in improved predictive modeling of film performance. In another project, resonant-ultrasonic techniques are applied to new piezoelectric materials for SAW devices, used extensively as oscillators in hand-held devices. These measurements probe fundamental damping mechanisms that underlie intrinsic acoustic loss and hence affect device performance.

In the Polymers Division, the electromagnetic properties of polymer composite films are investigated for applications in wireless communications. These materials can be used to construct RLC cells and de-coupling power planes integrated within chip substrates and printed circuit sub-assemblies. Current technologies utilizing discrete components cannot provide adequate solutions at frequencies above $1 \mathrm{GHz}$ that are critical for the high-speed electronics needed by wireless technologies. In partnership with the National Center for Manufacturing Science and industry, a collaborative research consortium was organized to address this inherent problem. NIST staff contribute materials science expertise, and specialized test vehicle designs and procedures for dielectric testing of high-k polymer composite films at microwave frequencies.

\section{Contact Information: Terrell Vanderah}




\section{Computational Studies of Ferroelectrics and Dielectrics}

\section{Benjamin P. Burton and Eric Cockayne}

Ceramic compounds with exploitable ferroelectric, dielectric, or magnetic properties are widely used in technical applications such as actuators, transducers, and dielectric resonators. We are using first-principles calculations to elucidate the roles of cation order-disorder and ferroelastic phenomena in dictating the phase relations and physical properties of these technologically important materials.
Ferroelectric, dielectric, magnetic, and transport properties of ceramics are typically sensitive functions of the state of cation order. Therefore, First-Principles Phase Diagram (FPPD) calculations are used to predict cation ordering phenomena, physical properties, and how these vary with chemical composition. Critical experiments are performed to test the predictions. An additional technical objective is to benchmark various FP techniques that are used to calculate physical properties (e.g. dielectric constant), and the formation energies on which FPPD calculations are based.

The intended outcome is to predict ordering behavior in complex technologically important oxide systems, with the objectives of: (1) minimizing the experimental work necessary to elucidate phase relations; (2) optimizing theoretical techniques; (3) optimizing processing strategies for these materials; (4) predicting the existence of new, technically important ordered phases; and (5) predicting how physical properties vary as functions of composition, temperature, and heat treatment.

The inclusion of degrees of freedom, derived from ionic motion, in the first-principles models will facilitate simulation and physical understanding of important properties. For example, the ferroelastic transitions in PZT, experimentally associated with strong piezoelectricity, can be simulated as a function of temperature, composition and stress. Molecular dynamics methods, with time-varying external fields, can be used to model the dielectric properties as a function of frequency in systems of microwave interest such as $\mathrm{CaTiO}_{3}-\mathrm{CaAl}_{1 / 2} \mathrm{Nb}_{1 / 2} \mathrm{O}_{3}$.

We have addressed several issues important to the processing and application of electronic ceramics and accomplished several advances in the modeling of these materials.

The different roles of long-range Coulomb interactions and short-range $\mathrm{Pb}-\mathrm{O}$ interactions in stabilizing cation ordered perovskite derivatives have been elucidated. The phases studied include Ba perovskites and disordered phases in the corresponding $\mathrm{Pb}$ perovskites. A first-principles based model of cation ordering in $\mathrm{PbMg}_{1 / 3} \mathrm{Nb}_{2 / 3} \mathrm{O}_{3}$ has been formulated and used to predict the ground-state ordered structure and an intermediate temperature phase with partial order. The intermediate $\mathrm{T}$ phase is ordered in a way that is clearly related to the shortrange order which is observed experimentally. The predicted ground-state phase has yet to be synthesized

A preliminary first-principles model of cation ordering in $\left[\mathrm{Na}_{1 / 2} \mathrm{Bi}_{1 / 2}\right] \mathrm{TiO}_{3}$ has also been formulated, and the ground-state ordered structure was successfully predicted. As observed experimentally, the predicted pseudocubic structure has a primitive cell with doubled cell constants in all three (x,y and z) Cartesian directions.

We have obtained first-principles results on phonon modes and static dielectric constants for $\mathrm{CaTiO}_{3}$ and $\mathrm{Ca}\left[\mathrm{Al}_{1 / 2} \mathrm{Nb}_{1 / 2}\right] \mathrm{O}_{3}$. The calculations show that the large dielectric constant in $\mathrm{CaTiO}_{3}$ arises primarily from a set of low-frequency vibrations in which the cations move in a direction opposite that of the oxygen ions. The corresponding modes in $\mathrm{Ca}\left[\mathrm{Al}_{1 / 2} \mathrm{Nb}_{1 / 2}\right] \mathrm{O}_{3}$ involve $\mathrm{Al}$ and $\mathrm{Nb}$ moving in opposition to $\mathrm{Ca}$, which suppresses the dielectric constant. We relate the differences in dielectric behaviors to differences in the electronic structures, namely the larger band gap in $\mathrm{Ca}\left[\mathrm{Al}_{1 / 2} \mathrm{Nb}_{1 / 2}\right] \mathrm{O}_{3}$.

A working group has been formed to develop a Unified Effective Hamiltonian theory for modeling systems that exhibit both cation order-disorder and ferroelastic transitions. The first workshop was held in June 2000. Group members attending were B.P. Burton and E. Cockayne (NIST); K.M. Rabe (Rutgers); L. Bellaiche (U. Arkansas); A. Fillipeti (UCSB); G. Ceder and A. Van der Ven (MIT); Mark Asta (Northwestern); David Singh (NRL); and W.A. Smith (ONR).
Contributors
1. Levin (University of Maryland) and T. A. Vanderah, Ceramics Division G. Ceder and A. Van der Ven (MIT); L. Bellaiche (Univ. Arkansas); J. Petzelt (Czech Academy of Sciences) 


\section{Dielectric Oxides for}

\section{Wireless}

\section{Communications}

\author{
T.A. Vanderah
}

Every modern commercial wireless communication system incorporates dielectric oxide ceramics with unique electrical properties as critical elements. The commercial competitiveness of next-generation devices depends on new ceramics with improved properties and/or reduced processing costs. Experimental phase equilibria determination integrated with systematic chemistry-structure-property studies contribute to the fundamental understanding of these ceramics. In addition, the existence of potentially useful phases and phase assemblages in selected oxide systems is revealed.
Dielectric oxide ceramics with unique electrical properties are used to fabricate a variety of components in cellular communications circuits that store, filter, and/or transfer electromagnetic energy with minimal loss (e.g., resonators, bandpass filters, circulators). The required properties for these ceramics include high dielectric constant, minimal dielectric loss, and essentially zero temperature dependence of relative permittivity. Knowledge of the materials science underpinning the appearance of these useful properties does not exist, hence all ceramic systems currently in use were empirically "discovered" and developed. The absence of practical theoretical guidance has been identified by U.S. industry as the most important R\&D materials issue for this class of electronic materials with regards to global commercial competitiveness (Workshop on Materials and Measurements for Wireless Communications, J. Res. NIST 101(6), 797-802 (1996)). Fundamental understanding of the chemical and structural basis of dielectric performance at wireless frequencies is needed to reduce the processing costs of existing ceramics, and to improve the design and discovery of next-generation materials.

This research activity includes experimental studies of phase equilibria and chemistry-structure-property relations of complex oxide systems containing one or more components or compounds that exhibit potentially useful dielectric properties. Systems are selected for study on the basis of empirical knowledge, results from integrated computational studies, and/or information from industry regarding next-generation needs. In addition to revealing the existence of new, possibly useful materials, knowledge of phase equilibria relations is important because all ceramic components are processed as mixtures to achieve "compensation", i.e., a net overall zero temperature coefficient. The technical work is multi-disciplinary and includes synthesis and crystal growth; structural analysis by $\mathrm{X}$ ray, electron, and neutron diffraction; determination of phase relations; characterization (via collaborative efforts) of dielectric properties at wireless frequencies; and measurement of vibrational spectra.

Recent results include completed studies of the $\mathrm{AO}-\mathrm{Al}_{2} \mathrm{O}_{3}$ $\mathrm{Nb}_{2} \mathrm{O}_{5}$ phase diagrams, where $\mathrm{A}=\mathrm{Mg}, \mathrm{Ca}, \mathrm{Sr}$, or $\mathrm{Ba}$. Using comparisons of polarizabilities and crystal chemistries, these systems were chosen as potential hosts of compounds similar to the costly but useful tantalum-based ceramics needed for high-frequency $(>2 \mathrm{GHz}$ ) base station resonators. The $\mathrm{MgO}$ system contains no ternary compounds. In the $\mathrm{CaO}$ system, the single ternary compound $\mathrm{Ca}_{2} \mathrm{AlNbO}_{6}$ exists, and was found to form a stable temperature-compensated mixture with $\mathrm{Ca}_{3} \mathrm{Nb}_{2} \mathrm{O}_{8}$. The dielectric properties of this potentially useful mixture were characterized at $5-7 \mathrm{GHz}$. In the $\mathrm{SrO}$ system three ternary compounds were found - two new compounds, $\mathrm{Sr}_{4} \mathrm{AlNbO}_{8}$ and $\mathrm{Sr}_{5.7} \mathrm{Al}_{0.7} \mathrm{Nb}_{9.3} \mathrm{O}_{30}$, in addition to the known double perovskite, $\mathrm{Sr}_{2} \mathrm{AlNbO}_{6}$. The $\mathrm{BaO}$ system was found to contain a new, nonferroelectric compound,

$\mathrm{Ba}_{5.75} \mathrm{Al}_{0.75} \mathrm{Nb}_{9.25} \mathrm{O}_{30}$, with the tetragonal tungsten bronze (TTB) structure. In addition, the TTB-type solid solution occurring on the $\mathrm{BaO}-\mathrm{Nb}_{2} \mathrm{O}_{5}$ binary was found to extend into the ternary region, dissolving up to 3 percent mol fraction $\mathrm{Al}_{2} \mathrm{O}_{3}$.

In another recently completed study, the effects of cation ordering on dielectric properties were isolated from chemical composition by investigating three polymorphs of $\mathrm{Ca}\left[\mathrm{Ca}_{1 / 3} \mathrm{Nb}_{2 / 3}\right] \mathrm{O}_{3}$. The three phases feature different arrangements of the $\mathrm{Ca}^{2+}$ and $\mathrm{Nb}^{5+}$ cations on the B-sites of the perovskite structure. Dielectric measurements at frequencies above $1 \mathrm{GHz}$ revealed a systematic dependence of the properties on the type of cation ordering. In particular, the structure with 2:1 ordering exhibited a lower dielectric constant and a significantly more negative temperature coefficient of resonance frequency than the structures with $1: 1$ and newly described $k=1 / 4[111]^{*}$ ordering. Rietveld refinements of structural models for the three $\mathrm{Ca}_{4} \mathrm{Nb}_{2} \mathrm{O}_{9}$ polymorphs were conducted using $\mathrm{X}$-ray and neutron powder diffraction data to elucidate structural details that could be correlated with the changes in dielectric properties. The most significant crystalchemical difference between the three polymorphs was in the coordination environment about $\mathrm{Nb}^{5+}$. Analysis of the refined bond distances indicated increasing average distortion of the $\mathrm{Nb}$ coordination sphere in going from the $1: 1$ to the $\mathrm{k}=1 / 4[111]^{*}{ }_{\mathrm{c}}$ to the $2: 1$ ordered structure. The increased fraction of strongly compressed $\mathrm{Nb}-\mathrm{O}$ bonds in the $2: 1$ structure associated with the large distortion was correlated with the decrease in dielectric constant and more negative value of temperature coefficient of the resonant frequency obtained for this polymorph. Raman spectra obtained for the three polymorphs exhibited differences that were consistent with the observed structural chemistry.
Contributors

and

Collaborators
D. Minor, W. Wong-Ng, J.Y. Chan, B.P. Burton, (Ceramics Division) R.D. Shull, Metallurgy Division), R.G. Geyer, EEEL; J.E. Maslar, CSTL, I. Levin, R. Coutts, Univ. Maryland; E. Cockayne, Catholic Univ., T. Lindsey, Appalachian State Univ., V. Miller, Rider Univ., T. Negas and S.Bell, TRAK Ceramics, Inc. 



\section{X-ray Characterization}

Synchrotron radiation sources provide intense beams of X-rays for leading-edge research in a broad range of scientific disciplines. The Synchrotron Radiation Program at NIST/MSEL includes the development and operation of beam stations at the National Synchrotron Light Source (NSLS) at Brookhaven National Laboratory, and at the Advanced Photon Source (APS) at Argonne National Laboratory. NIST is a partner at the APS with the University of Illinois at Urbana/Champaign, Oak Ridge National Laboratory, and UOP, in a collaboration called UNICAT. The emphasis is on microstructure characterization, where NIST scientists and researchers from industry, universities and other government laboratories perform state-of-the-art measurements on advanced materials.

Scientific studies currently underway include microstructure characterization of ceramics and plasma-sprayed ceramic coatings, crystal perfection of basic and applied materials, microstructure evolution as a function of deformation, and the atomic-scale and molecular-scale structures at surfaces and interfaces.

Active programs at the NSLS include the operation of the X23A2 beam station where highly monochromatic $\mathrm{X}$ rays in the energy range $4.9 \mathrm{keV}$ to over $30 \mathrm{keV}$ are provided for $\mathrm{X}$-ray absorption fine structure (XAFS) and diffraction anomalous fine structure (DAFS) measurements. These techniques enable the solution, for example, of the microscopic structures of technologically important strained thin films and rare earth doping of ceramic matrix composites. Another significant program at the NSLS is the operation of the soft-X-ray materials end station on beam line U7A, where X-ray photoemission spectroscopy and X-ray absorption spectroscopy are used to study the structure and chemistry of surfaces and bulk materials either in vacuum or under atmospheric reaction conditions. Recent studies include in situ catalyst characterization and an investigation of magnetic hard disk lubricant chemistry.

The first UNICAT APS beam line became operational November 1, 1999. It incorporates the newest technology, which enables NIST scientists to significantly improve our ultra-small-angle $\mathrm{x}$ ray scattering, in situ X-ray topography, real-time $\mathrm{X}$-ray microscopy, and X-ray absorption fine structure (XAFS) capabilities. It also offers opportunities for cutting-edge experiments in structural crystallography and time-resolved scattering, surface / interface scattering, diffuse scattering, and magnetic scattering. We anticipate extending our present portfolio of characterization capabilities to include an even wider range of materials measurements.

Experimental capabilities include:

- The brilliance at the APS makes it possible for the first time to monitor surfaces and interfaces in situ during MBE or CVD;

- Our sensitivity is increased by a factor of 100 in ultrasmall-angle $\mathrm{x}$-ray scattering from ceramics, coatings, biological materials and polymers;

- Resolution for imaging of defects in semiconductor crystals, window and dome materials, and superconducting crystals reach approximately $1 \mu \mathrm{m}$;

- Time resolved structural studies during melting or phase transitions in crystalline materials are now possible;

- Diffuse X-ray scattering can be used to determine structures and lattice imperfections in ceramics, metals, semiconductors, and superconductors;

- An extension of ultra-small-angle X-ray scattering (USAXS) into USAXS imaging is currently under development.

Future opportunities for development include the possibility of becoming a part of UNICAT-II: a synchrotron X-ray micro-beam capability for probing materials at the sub-micrometer level. High-throughput parallel detection X-ray diffraction and X-ray fluorescence on this instrument would offer a unique opportunity for combinatorial materials science. Additional advantages include the possibility of characterizing sub-micrometer strain and stress and selected interface structures. Commissioning exercises on a prototype instrument are currently underway to evaluate the final design.

Certification of Standard Reference Materials(SRMs) is an important aspect of the $\mathrm{x}$-ray characterization research. These materials are critical to the calibration of $x$-ray diffractometers found in most laboratories. Providing SRMs to the technical and scientific communities is a NIST responsibility coordinated with American constituents and other national laboratories. 


\section{Characterization of Infrastructure Cements}

Like many ceramics, cement and concrete display complex performance and reliability variations, related to their processing and service-life conditions, as given by the morphology and composition of cement and additives, and by the hydration parameters and environment. The hydrogenous, uncertain, nature of the binding gel hydration product further complicates attempts to improve cement and concrete reliability as needed, for example, in the nation's highway infrastructure. Here, small-angle scattering is used to elucidate the significant process-microstructure-property relationships.
Improvement in the performance and reliability of cements and concretes used in the nation's infrastructure (particularly bridges) requires more accurate models to predict the evolution of cement and concrete properties during hydration for the range of environments and additives used. This project applies small-angle neutron scattering (SANS) and ultra-small-angle $x$ ray scattering (USAXS) to characterize the microstructures of hydrating cements. The studies have explored the separate effects on cement hydration of adding silica fume (SF) and coal fly ash (CFA), each in increasing use as relatively inexpensive additives intended to enhance cement and concrete durability. Present technological interest lies in combining SF and CFA additives to optimize the durability of concrete structures. Thus, a ternary study is underway to link cement microstructure evolution during hydration to the quantity, morphology and composition of a range of SF/CFA combinations.

Our SANS and USAXS studies have focussed on an a priori determination of representative density and formula data for the main strength-giving cement hydration product, amorphous calcium-silicate-hydrate (C-S-H) gel, as hydration proceeds. Uncertainty in the composition and nanoscale structure of the amorphous $\mathrm{C}-\mathrm{S}-\mathrm{H}$ gel has presented a major obstacle in building reliable cement hydration models, as well as in interpreting the small-angle scattering experiments.

Discrepancies in the published results of SANS and SAXS studies have also complicated the situation. Taking a standard 28 day hydration time as a starting point, we have used $\mathrm{H}_{2} \mathrm{O} / \mathrm{D}_{2} \mathrm{O}$ and $\mathrm{CH}_{3} \mathrm{OH} / \mathrm{CD}_{3} \mathrm{OH}$ saturated-pore-fluid SANS contrast-variation experiments, together with direct comparisons of absolute-calibrated SANS and USAXS data, to obtain C-S-H formulae and densities for selected cement mixes.

While absolute calibration of SANS data utilizes secondary standards, absolute SAXS intensity calibration is carried out via primary methods. The large dynamic intensity range of our USAXS instrument permits direct measurement of the smallangle scattering intensity normalized to that of the primary beam. This has established that no discrepancy exists between our SANS and USAXS results. Furthermore, we have established, for the first time, that the C-S-H forming in the pore space between the cement clinker grains has a solid density and composition close to that postulated for so-called D-dried cement. This means a C-S-H formula of $(\mathrm{CaO})_{1.7} \cdot\left(\mathrm{SiO}_{2}\right) \cdot\left(\mathrm{H}_{2} \mathrm{O}\right)_{1.4}$ and a solid density of $2.7 \mathrm{~g} \mathrm{~cm}^{-3}$ and describes the solid form of $\mathrm{C}-\mathrm{S}-\mathrm{H}$ assumed in nitrogen sorption studies of cement. Thus, any additional $\mathrm{H}_{2} \mathrm{O}$ units associated with $\mathrm{C}-\mathrm{S}-\mathrm{H}$ in a saturated system are loosely-adsorbed surface layers that can be investigated by inelastic scattering methods. Finally, the studies have established the existence of a finelydivided $\mathrm{Ca}(\mathrm{OH})_{2}$ phase that forms to a varying degree within the nanoscale $\mathrm{C}-\mathrm{S}-\mathrm{H}$ gel, depending on the nature of the cement mix. Such experiments can provide valuable input information for building numerical dynamic models for cement hydration and durability.

Fig. 1 shows a comparison of absolute-calibrated SANS and USAXS data versus the scattering vector, $Q$, where $Q=(4 \pi / \lambda)$ $\sin \left(\phi_{\mathrm{s}} / 2\right)$ and $\phi_{\mathrm{s}}$ is the angle of scatter. The USAXS/SANS ratio for scattering from the $\mathrm{C}-\mathrm{S}-\mathrm{H}$ gel/pore interface is consistent with the above interpretation for the C-S-H gel.

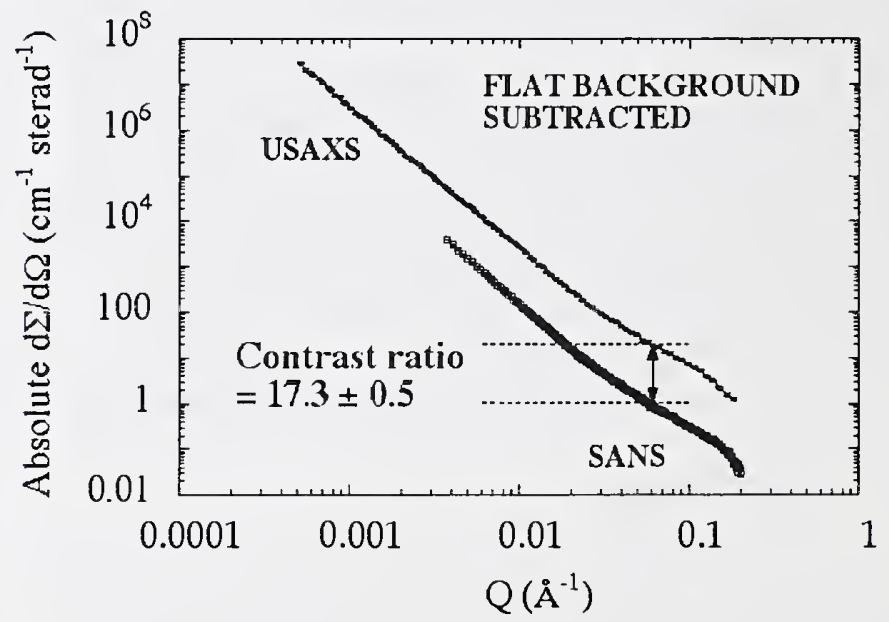

Fig. 1. Absolute-calibrated SANS and USAXS data versus the scattering vector for scattering from the C-S-H gel/pore interface.
Contributors and Collaborators
D.A. Neumann (NIST Center for Neutron Research) and E.J. Garboczi (Building Materials Division, BFRL); R.A. Livingston and W. Bumrongjaroen (FHWA); J.J. Thomas and H.M. Jennings (Northwestern University) 
EXAFS Measurement of

\section{Bond-length Strain in Thin $\mathrm{Gd}_{2} \mathrm{O}_{3}$ Films on $\mathrm{GaAs}(001)$}

The extended x-ray absorption fine structure (EXAFS) technique was used to measure the bond length in a $2.3 \mathrm{~nm}$ thin $\mathrm{Gd}_{2} \mathrm{O}_{3}$ film grown epitaxially on $\mathrm{GaAs}(001)$. The $\mathrm{Gd}-\mathrm{O}$ bond length is determined to be $0.2390 \pm 0.0013 \mathrm{~nm}$, which is a +2.7 $\pm 0.6 \%$ bond-length strain relative to that in bulk $\mathrm{Gd}_{2} \mathrm{O}_{3}$ powder. We develop a model for the thin film relating macroscopic strain to microscopic atomic displacements.

\section{E.J. Nelson and J.C. Woicik}

The long search for a stable, passivating surface oxide for GaAs possibly has ended with the development of $\mathrm{Gd}_{2} \mathrm{O}_{3}$ films. These epitaxially grown films are single crystal with a low number of defects and interfacial states, and they successfully have been used to fabricate metal-oxide-semiconductor structures on GaAs. We studied a $2.3 \mathrm{~nm}$ thin $\mathrm{Gd}_{2} \mathrm{O}_{3}$ film grown epitaxially on the $\mathrm{GaAs}(001)$ substrate at Bell Laboratories, Lucent Technologies.

EXAFS experiments were performed at NIST beamline X23-A2 at the National Synchrotron Light Source. The Gd L $\alpha$ fluorescence yield around the Gd LIII edge $(\mathrm{h} v=7243 \mathrm{eV})$ was monitored using a single-element SiLi detector. EXAFS data were recorded with the sample surface normal $\mathbf{n}$ either parallel $(\varepsilon \| \mathbf{n})$ or perpendicular $(\varepsilon \perp \mathbf{n})$ to the polarization vector $\varepsilon$ of the synchrotron radiation. In addition, EXAFS data from a pure $\mathrm{Gd}_{2} \mathrm{O}_{3}$ powder were collected in transmission, to determine the EXAFS phase and amplitude standards for the Gd-O bond length.

Figure 1 shows the $k^{2}$-weighted Gd LIII edge EXAFS from the $\mathrm{Gd}_{2} \mathrm{O}_{3}$ powder. Also shown are the EXAFS from the $\mathrm{Gd}_{2} \mathrm{O}_{3}$ epitaxial film recorded in the two polarizations. The frequency of the EXAFS oscillations and, therefore, the Gd-O bond length $r$ in the film is increased significantly relative to the powder. The best fits produce $r=0.2391 \pm 0.0017 \mathrm{~nm}$ for $\varepsilon \| \mathbf{n}$ and $r=0.2389 \pm$ $0.0019 \mathrm{~nm}$ for $\varepsilon \perp \mathbf{n}$, so together we determine an average Gd-O bond length $r=0.2390 \pm 0.0013 \mathrm{~nm}$. This is a $+0.0063 \pm 0.0013$ $\mathrm{nm}$ or $+2.7 \pm 0.6 \%$ increase relative to the $0.2327 \mathrm{~nm}$ bond length in bulk $\mathrm{Gd}_{2} \mathrm{O}_{3}$.

In addition, the data from the two polarizations are identical within the noise throughout the entire $k$ range. This indicates that the first and second shell local structures are similar along the [110] and [110] directions of the strained $\mathrm{Gd}_{2} \mathrm{O}_{3}$ film; therefore, the strains along [110] and [-110] are equal, as suggested by the crystal symmetry of $\mathrm{Gd}_{2} \mathrm{O}_{3}$ (see Figure 2).

We developed a simple model for the strained film, with a unique Poisson distortion that matches the [001] and [-110] axes of $\mathrm{Gd}_{2} \mathrm{O}_{3}$ with the [110] and [1-10] axes of the $\mathrm{GaAs}(001)$ surface. This model assumes that the microscopic displacements of the atoms are proportional to the macroscopic changes in the lattice

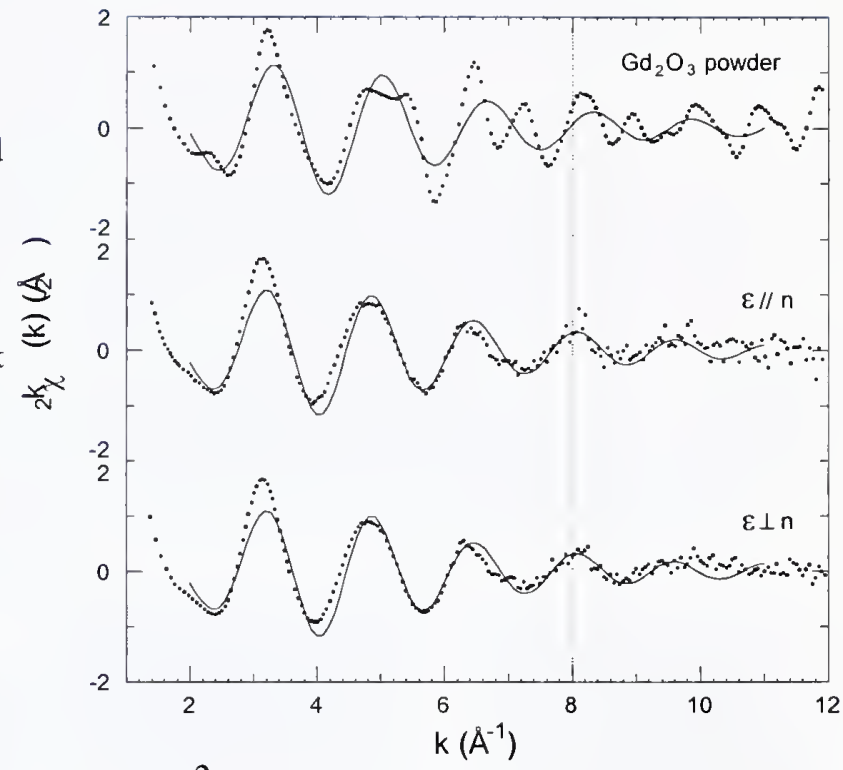

Figure 1. $k^{2}$-weighted Gd LIII edge EXAFS data from the bulk standard $\mathrm{Gd}_{2} \mathrm{O}_{3}$ powder (top). Also shown are the EXAFS from the thin $\mathrm{Gd}_{2} \mathrm{O}_{3}$ film on $\mathrm{GaAs}(001)$, with the Xray polarization vector aligned parallel (middle, $\varepsilon \| \mathbf{n}$ ) and perpendicular (bottom, $\varepsilon \perp \mathrm{n}$ ) to the $\mathrm{GaAs}(001)$ surface normal. Lines are best fits to the first-shell component.

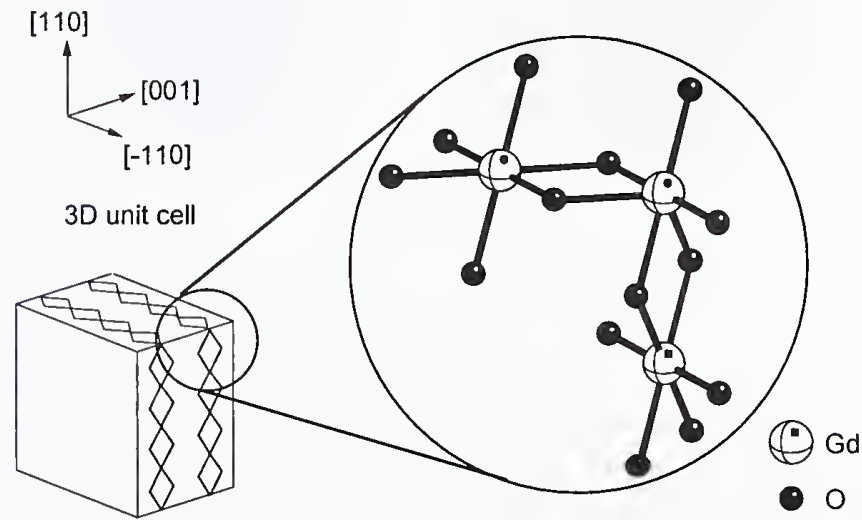

Figure 2. Local environment of the Gd atoms (right). Each $\mathrm{Gd}$ atom is surrounded by a distorted octahedron of $6 \mathrm{O}$ atoms. The Gd-O bonds form "diamond" chains along the [110] and [-110] directions of the unit cell (left).
Contributors and
M. Hong, J. Kwo, and J. P. Mannaerts

Bell Laboratories, Lucent Technologies

Murray Hill, NJ 07974 


\section{Materials}

\section{Characterization Using}

\section{X-ray Topography}

\section{David R. Black and Harold E. Burdette}

The NIST X23A3 high-resolution X-ray topography facility at the NSLS is the only dedicated monochromatic instrument of its type in this country. It is used in studies of a wide range of basic and applied materials. In the coming year, we will commission our new monochromatic $X$-ray topography and imaging facilities on UNICAT sector 33 at the APS.

\section{X-ray Topography of Protein Crystals Grown in Space}

One of the outstanding questions in protein crystal growth is what affect does gravity have on the growth process. The crystallization of proteins can be strongly affected by gravity through sedimentation and convection. Will the elimination of these processes, through growth in microgravity, produce better quality crystals? To answer this question a comparison has been made between ribonuclease crystals grown on the space shuttle and control crystals grown at the same time on Earth. Comparisons of X-ray topographs recorded from Earthgrown and space-grown crystals showed that the space-grown crystals were of higher crystal perfection than the ground grown crystals can be seen below.
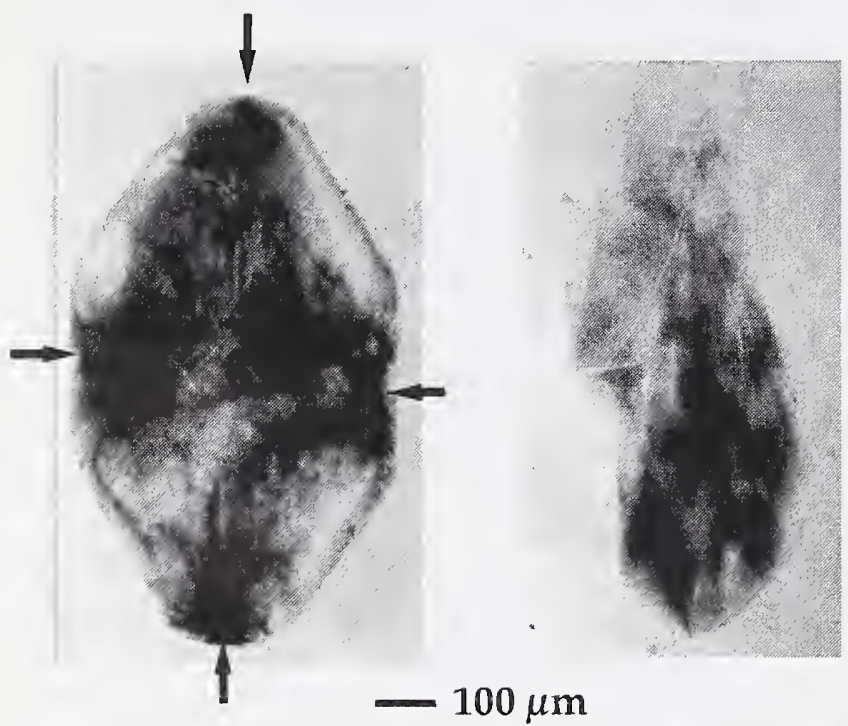

$100 \mu \mathrm{m}$

(006) X-ray diffraction topographs of a space-grown crystal on the left, and an earth-grown crystal on the right. The space crystal has symmetry about the vertical and horizontal axes consistent with growth sectors. As the Bragg angle is changed, the four dark regions indicated together migrate toward or away from the center of the crystal, consistent with a central nucleation point followed by homogeneous growth. The microstructure of the earth crystal is less well defined consistent with a higher defect density.

\section{Characterization of Surface Damage in Sapphire Bend Bars}

X-ray topography has identified strength limiting surface damage in sapphire bend bars. A group of 39 bars, which had been fabricated to identical specifications, was found to contain two distinct microstructures as shown below. The Type 2 microstructure is typical of a nearly damage-free surface, where the dislocation structure is visible and no residual fabrication damage is observed. The Type 1 microstructure is typical of a damaged surface, where no underlying dislocation structure is visible and the linear nature of the surface defects is consistent with damage caused by a grinding operation that has not been completely removed by subsequent polishing. The average fracture strength, in four point bending, of the Type 1 bars was less than $70 \%$ of the strength of the Type 2 bars.
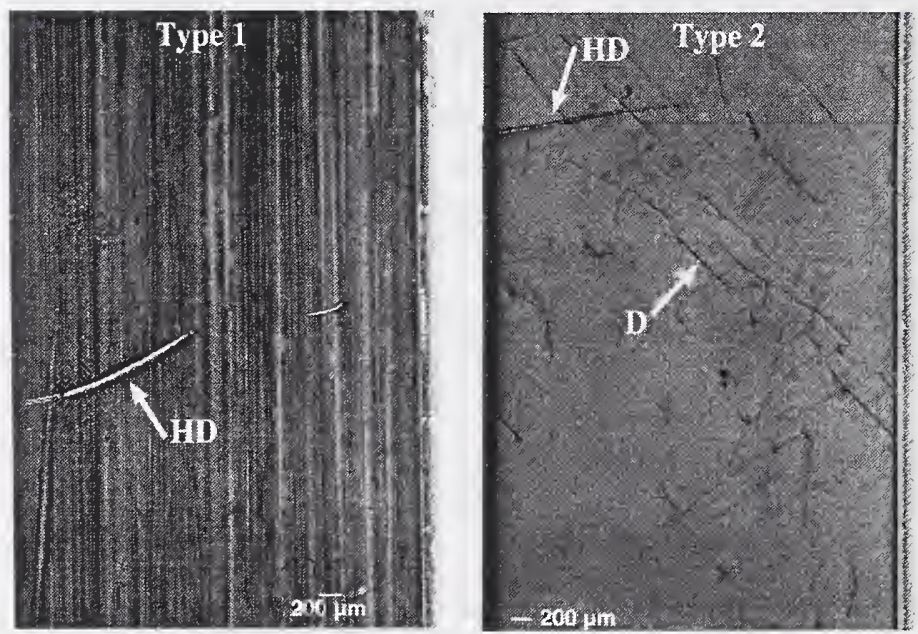

Representative topographs of the Type 1 and Type 2 bars. Dislocations (D) are visible in the Type 2 bar but are not visible in the Type 1 bar. The damage in the Type 1 bar obscures the underlying microstructure. Both types of bar show evidence of inadvertent damage (HD). 


\section{Powder Diffraction}

\section{Standards}

\author{
James P. Cline
}

The defining virtue of the powder diffraction technique is that it allows the user to probe all crystallographic reflections of a given d space range with a single scan. However, the parafocusing geometry of laboratory X-ray powder diffractometers offer data beset by a complex error function. Characterization of this error function, which is critical to the determination of accurate crystallographic and microstructural parameters, is one of the prime functions of NIST Standard Reference Materials (SRMs) for powder diffraction. Additional SRMs are used for quantitative analysis of multiphase mixtures.
While the $d$ spacing and intensities of powder diffraction patterns can be calculated accurately from the crystal structure, the data within experimental patterns themselves embody aberrations specific to the powder diffractometer used for the measurement. The effect of these aberrations, convoluted with the profile of the radiation source, is generally referred to as the instrument profile function (IPF). Requisite to accurate characterization of the IPF is a standard which, due to its microstructure, imparts no discernable broadening to the diffraction profiles, and for which the lattice parameter is accurately determined. Production of a NIST Standard Reference Material, SRM, which meets these two criteria presents both a material and a metrological challenge.

The primary NIST line position SRM for powder diffraction, SRM 640b, silicon powder, was renewed this year as SRM 640 c. Concurrently, the line profile SRM 660, lanthanum hexaboride, was renewed as SRM 660a. The line positions of both SRMs $640 \mathrm{~b}$ and 660 were certified to a few parts in $10^{5}$, which is entirely suitable for conventional data analysis techniques. However, to remain relevant for modern data analysis methods, it was necessary to improve the accuracy of the certified values by an order of magnitude. Furthermore, it was also desired to address the microstructure of both SRMs to render SRM 640c suitable for use as a line profile standard and to remove the slight amount of strain broadening known to affect SRM 660.

The uniformity of the ultra high purity, intrinsic silicon boules used as the feedstock for SRM $640 \mathrm{c}$ was verified prior to comminution. A total of 70 measurements covering the longitudinal and radial boule directions were performed on the NIST lattice comparison apparatus. The relative lattice variation of the input material implied from these measurements was $\pm 4 \times 10^{-8}$ (95\% confidence level).

Comminution of the silicon boules was done via a jaw crusher followed by a jet mill. The powder was then annealed under gettered argon at $1000^{\circ} \mathrm{C}$ for two hours. SRM 660a was prepared via a solid state process, jet milled, and annealed. Both SRMs offer a narrow particle size distribution; the mean of SRM $640 \mathrm{c}$ is $\cong 4.9 \mu \mathrm{m}$ while that of while that of SRM $660 \mathrm{a}$ is $\cong 8.8 \mu \mathrm{m}$. However, the powder of SRM $640 \mathrm{c}$ consists of single crystal particles while that of SRM 660 a consists of aggregates of crystallites.

The certification measurements were performed on a diffractometer built for first principles lattice parameter measurements. The metrologically intractable components of the IPF associated with a conventional diffractometer, i.e., flat specimen, specimen position, beam penetration, and axis centration errors, were eliminated with the preparation of a parallel incident beam. The preparation optic also transmitted the $\mathrm{K}_{\alpha 1} / \mathrm{K}_{\alpha 2}$ emission spectrum of copper without distortion establishing the linkage to the International System of Units (SI). An auto-calibrating optical encoder that resulted in an angular measurement uncertainty of approximately $9.7 \times 10^{-7}$ rad was used for in situ angle measurement. The $2 \theta$ zero error was eliminated by collecting profile data on either side of the incident beam. Diffracted beam analysis was performed with a high-resolution equatorial Soller collimator. Axial divergence of the incident and diffracted beam was limited by Soller slits.

The IPF of the parallel beam diffractometer was rigorously modeled using the Fundamental Parameters Approach to obtain the "true" profile positions. The refinement strategy constrained certain parameters, such as the one describing the transmission function of the equatorial Soller analyzer, across the entire pattern. Parameters specific to each profile, such as $2 \theta$ position, were refined independently. Results were considered as a plot of lattice parameters determined for each profile $v s$. 20. With ideal instrumentation and data analysis, the lattice parameters do not vary across $2 \theta$. The most problematic aspect of the IPF was the axial divergence, which led to deviations at high and low angle. Certified values were those obtained from profiles of the 80 to 130 degrees $2 \theta$ region wherein the effects of axial divergence are minimal.

The lattice parameters of SRM 640c were certified to an accuracy of 4 parts in $10^{6}$, those of SRM 660 a were certified to an accuracy of 5 parts in $10^{6}$. Furthermore, the preparation procedure of these SRMs resulted in microstructures which have virtually eliminated strain broadening in SRM 660a and will allow the use of SRM 640c as a line profile standard. 
Semiconductor and

\section{Electronic Materials}

\section{Evaluation}

\section{J.C. Woicik and E.J. Nelson}

We have developed a new experimental method by which sitespecific valence electronic structure may be obtained. It utilizes the spatial variation of the electric-field intensity that occurs near an $X$-ray Bragg reflection, together with highresolution valence-photoelectron spectroscopy, to determine the partial densities of occupied valence states of crystalline materials. Resolution of the anion and cation contributions to the GaAs valence band is demonstrated.
Spatially-resolved valence electronic structure

One of the most powerful experimental tools for examining the electronic structure of solids or films is photoelectron spectroscopy. Photoemission yields direct electronic information pertaining to the occupied valence-band density of states for many materials. Results have been used to establish the validity of complicated band-structure calc-ulations for metals, semiconductors, insulators, and alloys.

Typical photoemission measurements are performed with excitation sources that are assumed to be monochromatic plane waves. As the intensity of a plane wave is constant over the dimensions of a unit cell, standard photoemission measurements are unable to produce direct, site-specific valence information. However, such information is paramount for advancing our understanding of how chemical bonding results in the solid-state electronic structure.

Here we describe a new experimental method for obtaining site-specific valence electronic structure. It utilizes the spatial variation of the electric-field intensity that occurs within the vicinity of a crystal $\mathrm{X}$-ray Bragg reflection which is given by the superposition of the incident $\mathbf{E}_{\mathrm{O}}$ and reflected $\mathbf{E}_{\mathrm{h}} \mathrm{X}$-ray beams that travel with wave vectors $\mathbf{k}_{\mathrm{O}}$ and $\mathbf{k}_{\mathrm{h}}$, polarization vectors $\mathbf{e}_{\mathrm{o}}$ and $\mathbf{e}_{\mathrm{h}}$, and frequency $\square$ :

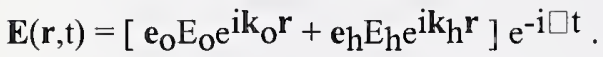

$\mathbf{k}_{\mathrm{O}}$ and $\mathbf{k}_{\mathbf{h}}$ are connected by the Bragg condition $\mathbf{h}=\mathbf{k}_{\mathbf{h}}-\mathbf{k}_{\mathrm{O}}$, where $\mathbf{h}$ is a reciprocal-lattice vector of the crystal. For the $\square$ polarization geometry of a symmetric reflection, this field squares to give the electric-field intensity at an arbitrary point $\mathbf{r}$ in space:

$I(r)=\left|E_{O}\right|^{2}[1+R+2 \sqrt{R} \cos (\square+\mathbf{h} \cdot \mathbf{r})]$.

is the phase of the complex-field amplitude ratio $E_{h} / E_{0}=$ $\sqrt{ } \mathrm{Re}^{\mathrm{i}} \square$, and $\mathrm{R}$ is the reflectivity function $\mathrm{R}=\left|\mathrm{E}_{\mathrm{h}} / \mathrm{E}_{\mathrm{O}}\right|^{2}$. With this method, we can position the maxima/minima of the electric field at any location within the crystalline-unit cell by experimentally varying the phase of the complex reflectivity function between $0<\square<\pi$. This is achieved by slightly varying either the sample angle or the photon energy within the natural width of the X-ray Bragg reflection.
Because the probability of emission of an electron from an atom in an external electric field is proportional to the electric-field intensity at the location of its atomic core (dipole approximation), by selectively positioning the electric-field intensity within the unit cell and recording highresolution valence-photoelectron spectra, spatially resolved components to the photoemission valence-band density of states may be directly obtained.

To illustrate the principle of the technique, Fig. 1 shows the partial density of states of the GaAs valence band obtained by decomposing the GaAs photoelectron valence-band spectra obtained under different electric-field conditions according to Eqn. 2. These spectra are compared to ab initio theoretical calculations of the Ga and As partial density of states. Remarkable qualitative agreement between the theoretical and experimental spectra is observed. The differences between the two electronic structures are due to the natural ordering of the $\mathrm{Ga}$ and $\mathrm{As} 4 \mathrm{~s}$ and $4 \mathrm{p}$ valence states, together with the solid-state bonding that has occurred between them.

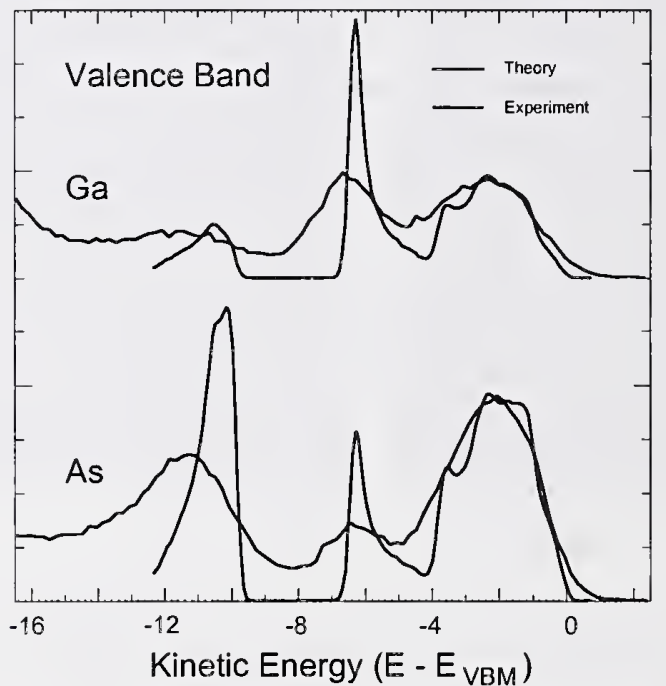

Fig. 1. Partial density of states of the GaAs valence band compared to ab initio theoretical calculations.

In the future, this method should provide useful information on the detailed electronic structure of more complex crystalline materials of technological importance.
Contributors and
T. Kendelewicz and P. Pianetta (Stanford University); M. Jain, L. Kronik, and

J.R. Chelikowsky (University of Minnesota) 


\section{Soft X-ray Surface Characterization}

\section{A. Fischer and S. M. Hsu}

We utilize polarized soft $x$-rays to probe the structure and molecular orientations nanometer thick layers on a variety of surfaces in partnership with industries and academic institutions.

Practical industrial problems are currently being investigated, such as model catalyst systems, polymer surfaces and their interfaces, magnetic hard disk surface chemistry, biomaterials, and high temperature superconductors.
Soft $X$-ray analysis of ultra-high molecular weight polyethylene subjected to wear contacts

Over half a million patients receive artificial joint replacements annually in the US and the dominant materials are ultra-high molecular weight polyethylene (UHMWPE) and CoCr alloy. There is a strong need to extend current durability significantly due to longer life expectancy. Wear and wear debris are thought to limit the current component life. The detailed mechanisms of wear and debris generation are not clearly understood. One of the difficulties is the lack of sensitive tools to examine UHMWPE microstructural and molecular orientation change due to contact sliding. Recently, highly cross-linked UHMWPE has been introduced which drastically reduces wear. Working in conjunction with the NIST Orthopaedic Wear Consortium and other companies, the near-edge X-ray absorption fine structure (NEXAFS) spectroscopy at our unique ultra-soft X-ray materials science end station at the NSLS was used to measure surface molecular orientations of various cross-linked UHMWPE samples (sterilized by gamma and electron irradiation) before and after wear. We also obtained retrieved samples from patients for comparison.

In general, highly cross-linked samples tend to orient or align with the wearing direction much less than the uncross-linked samples under identical treatment. Upon repeated cycles under high stress conditions, the highly cross-linked samples eventually reach the same orientation or alignment as the uncrosslinked samples. The surface molecular alignment is reduced by cross-linking. Another series of samples with different degrees of cross-linking was obtained by irradiating these samples from $30 \mathrm{kGy}$ to $100 \mathrm{kGy}$ radiation. These samples were then subjected to a knee simulator wear test with a defined machine direction. NEXAFS of these samples reveals a strong correlation of resistance to induced orientation with increased radiation dose (increased cross-linking) saturating at $75 \mathrm{kGy}$.

The NEXAFS measurements of molecular orientation of UHMWPE provide key insight into the wear mechanisms of a new generation of biomaterials and enable industrial partners to design better materials based on these measurements.

\section{Magnetic hard disk surface chemistry probed by NEXAFS}

Magnetic data storage density is doubling every eighteen month and the cost is coming down. The continuing advance in this technology depends on the ability of a monolayer of lubricant in conjunction with a thin layer of diamond like carbon protective coating to protect the magnetic layer from wear in the face o increasingly lower flight heights $(5 \mathrm{~nm})$ and higher speeds (40 $\mathrm{m} / \mathrm{s}$ ). Molecular assemblies with mixed molecular species have been explored by the Surface Properties Group in NIST in conjunction with Pennzoil through a CRADA. The ability to probe the surface chemistry at a nanometer scale is critical to lear how to design and assemble various molecules into a usefu technological concept. Current techniques such as AFM an SPMs are not suitable to probe liquid-like complex molecules with molecular weights in the thousands. Soft X-ray near edge fine structure spectroscopy (NEXAFS) is one technique that has sufficient chemical and surface $(10 \mathrm{~nm})$ sensitivity to prob monolayers on an engineering surface such as the supersmoot hard disks. The chemical bond specificity of the NEXAFS technique enables the differentiation of the carbon in Pennzaneß)( multiply cyclopentane polymer) from the carbon in the diamon like carbon overcoat and to isolate their chemical interaction Single component and multi-component films of one nanometer thick were successfully deposited onto various prototype magnetic hard disks and probed by NEXAFS. Pennzane ${ }^{\circledR}$ was found $t$ interact with the carbon overcoat forming a new $\mathrm{C}=\mathrm{C}$ bond. In three-component mixture, each individual molecular species shows no interaction with the carbon overcoat yet the mixture shows a complex interaction pattern involving all three components. This finding provides significant insight on the interaction an assembly of molecular structures at a monolayer level on a engineering surface such as a magnetic hard disk.

These observations agree with the observation of enhanced performance for multi-component molecular assemblies as compared to the individual components at equal thickness. This paves the way for more intelligent design of practical films for various applications.
Ming Shen (U MD/NIST), Dan Xiang (U MD/NIST)

S. Sambasivan (S.U.N.Y./Stony Brook), Aiguo Wang (Howmedica), Dave Schroeder (biomat), Les Gilbertson (Zimmer), Don McNulty (J\&J), Richard Gates (NIST), Selda Gunsel (Pennzoil) 
Synchrotron Beam Line Operation and Development
The Synchrotron Radiation Program at NIST/MSEL includes the development and operation of beam stations at the National Synchrotron Light Source (NSLS) at Brookhaven National Laboratory, and at the Advanced Photon Source (APS) at Argonne National Laboratory. The emphasis is on microstructure characterization, where NIST scientists, and researchers from industry, universities and other government laboratories perform state-of-the-art measurements on advanced materials.

\section{Gabrielle Long}

The UNICAT collaboration at the Advanced Photon Source, which includes NIST, the University of Illinois, Oak Ridge National Laboratory, and UOP Research Center, includes research techniques that require widely varying characteristics of the incident X-ray beam. To meet the technical demands of our diverse experimental portfolio, the UNICAT beam lines on Sector 33 exploit the brilliance of the APS source to deliver Xrays with high flux and with energy resolution better than or equal to the lifetime-broadened core-hole widths for all accessible $\mathrm{K}$ and $\mathrm{L}$ shells. The insertion device beam line, 33ID, became operational November 1, 1999, and as of November 1,2000 , will entertain independent investigators as well as collaborative access team members. The bending magnet beam line, 33-BM, is going into commissioning December 15, 2000 and is expected to become fully operational by the end of 2001 .

Ultra-small-angle X-ray scattering (USAXS) is the first of the NIST-responsible experiments to come on line. USAXS provides data in the usually inaccessible $Q$ range (where $Q=$ $(4 \pi / \lambda) \sin \theta, \lambda$ is the $x$-ray wavelength and $\theta$ is one-half the angle of scatter) down to $1.510^{-4} \AA^{-1}$. In that role, USAXS fills the gap between visible light scattering and pinhole small angle cameras. The UNICAT instrument, which has had nearly a year of full operation, includes several capabilities that make it one of the best in the world. It covers an unprecedented Q range out to $1 \AA^{-1}$; it can operate as an "effective pinhole" instrument, where the scattering data is not line-smeared; anomalous-USAXS can be used to uncover and quantify previously inseparable microstructures; and it supports the remarkable new technique of USAXS-imaging. In the past year, the USAXS instrument enjoyed the highest demand of any instrument on UNICAT, and it is the first instrument to be requested by independent investigators.

In the coming year, we are looking forward to commissioning three new NIST-responsible experiments: X-ray topography, Xray microtomography, and $\mathrm{X}$-ray absorption fine structure. The microtomography is a new capability which is expected to achieve a resolution of $0.3 \mu \mathrm{m}$ by a form of in-line near-field holography. This resolution is nearly an order of magnitude better than the current state of the art.
At the NSLS, we operate two beam lines: U7A and X23A2. Numerous improvements have been made to the ultra-soft Xray (U7A) Materials Science End Station over the past year. Continuous cleaning of the grating optical surfaces became a practical reality with the installation of a pair of special ion pumps and arranging a controlled leak of oxygen gas into the grating chamber. An energy-resolving electron yield detector was installed to enhance and improve the definition of the surface sensitivity of our NEXAFS measurements; this detector is fully operational and is a very significant improvement in sensitivity over the previous design. A new linear drive, that moves 100,000 times faster than our old drive was commissioned and is now in continuous use. In a Phase II SBIR, designs were finalized for the $\mathrm{I}_{0}$ four slit diode with visible light barrier. In addition, the mechanical setup and actuators for this detector were designed. Finally, the design and construction of ultra high efficiency $\mathrm{Si}(\mathrm{Li})$ and high purity Ge energy dispersive detectors with ultra-thin surface dead-layers is underway; its purpose lies in improving our X-ray detection sensitivity at energies below 1 $\mathrm{keV}$. This would be a very significant advance in the quantitative analysis of light elements performed in electron micro probes in many analytical and research laboratories in the United States.

The X23A2 beam line provides a stable, scanning, highly monochromatic X-ray beam in the energy range from 4.9 $\mathrm{keV}$ to over $30 \mathrm{keV}$. The $\mathrm{X}$-ray absorption fine structure (XAFS) spectroscopy done on this line is capable of probing: the short range order in crystalline and amorphous materials; one element at a time, and it provides information on the number, distance and chemical identity of the neighbors of the absorbing atom.
A.J. Allen, D.R. Black, H.E. Burdette, D.A. Fischer, R.D. Spal and J.C. Woicik (Ceramics Division); P.R. Jemian, H. Chen, H. Hong and P. Zschack (University of Illinois); J. Ilavsky (University of Maryland); G.E. Ice, J.Z. Tischler and B.C. Larson (Oak Ridge National Laboratory) and R. W. Broach (UOP Research) 
USAXS Characterization of Materials

\section{Andrew J. Allen and Gabrielle G. Long}

Ultra-small-angle X-ray scattering (USAXS) experiments supply data in the usually inaccessible $Q$ range (where $Q=$ $(4 \pi / \lambda) \sin \theta, \lambda$ is the $x$-ray wavelength and $\theta$ is one-half the angle of scatter) down to $1.5 \times 10^{-4} \AA^{-1}$. In that role, USAXS fills the gap between visible light scattering and pinhole small angle cameras. As one of the few small-angle instruments in the world for which a primary absolute calibration is available, the results from the NIST USAXS facility are quantitative rather than qualitative, which is of great importance to serious materials science research.
The ultra-small-angle X-ray scattering (USAXS) instrument on UNICAT sector 33 at the Advanced Photon Source provides data in the usually inaccessible scattering vector, $\mathrm{Q}$ range (where $Q=(4 \pi / \lambda) \sin \theta, \lambda$ is the $x$-ray wavelength and $\theta$ is one-half the angle of scatter) down to $1.510^{-4} \AA^{-1}$. This unusual instrument covers nearly 4 decades in $Q$, and 12 decades in linear dynamic range in X-ray intensity. In the past year, its function has been extended to the measurement of anisotropic USAXS and USAXS imaging.

To remove the intrinsic slit-smearing of the USAXS instrument, transverse crystal reflections orthogonal to the plane of the collimator and analyzer crystal reflections are introduced. The data in a single scan are associated with one azimuthal direction in the plane of the sample. Thus, the sample is measured repeatedly for different azimuthal orientations, where the resolution of the azimuthal rotation increments can be made almost vanishingly fine. Indeed, the anisotropic resolution of the new instrument can surpass that of conventional pinhole instruments with a $2 \mathrm{D}$ detector.

Studies of textured $\mathrm{Fe}_{2} \mathrm{TiO}_{5}$ have been carried out with reference to numerical model predictions based on the OOF program of NIST's CTCMS. Fig. 1 shows the anisotropic scattering associated with cracks perpendicular to a strong fiber- texture axis (strong maximum along the direction, B) and also a $2 \mathrm{D}$ random distribution of microcracks with planes parallel to the direction, $\mathbf{B}$ (maximum around equator perpendicular to $\mathbf{B})$.

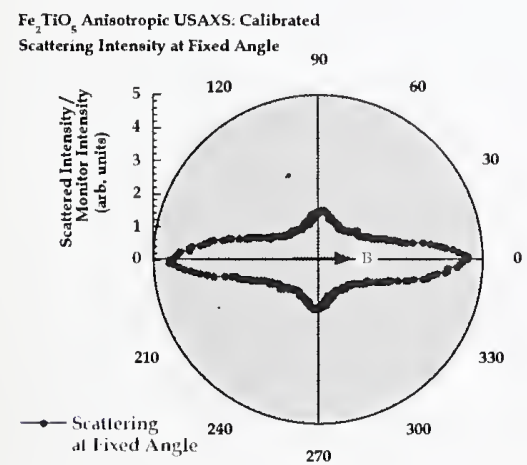

USAXS imaging is a completely new class of X-ray imaging technique that is remarkably sensitive to the microstructures within a scattering volume. In USAXS imaging, the analyzer is rotated to a specific angle and the selected X-rays are used to form an image of the sample. Since the only X-rays that contribute to the image are those produced by small-angle scattering, the image is a direct map of where the USAXS is originating from within the sample. Information on the sizes and shapes of the scattering objects can be obtained by comparing images produced at different scattering angles. Such information can be determined even when the scattering objects are smaller than the spatial resolution of the imaging process! The image contrast doesn't change during sample rotations about the scattering vector (vertical axis in the lab) and combining images from two such rotations has produced stereo USAXS images. In principle, a full 3D tomographic reconstruction should also be feasible. USAXS imaging should prove useful both as an independent imaging technique and as an important adjunct to USAXS generally. It is likely to find application to a broad range of materials problems in metal, ceramic, polymer and biological systems.

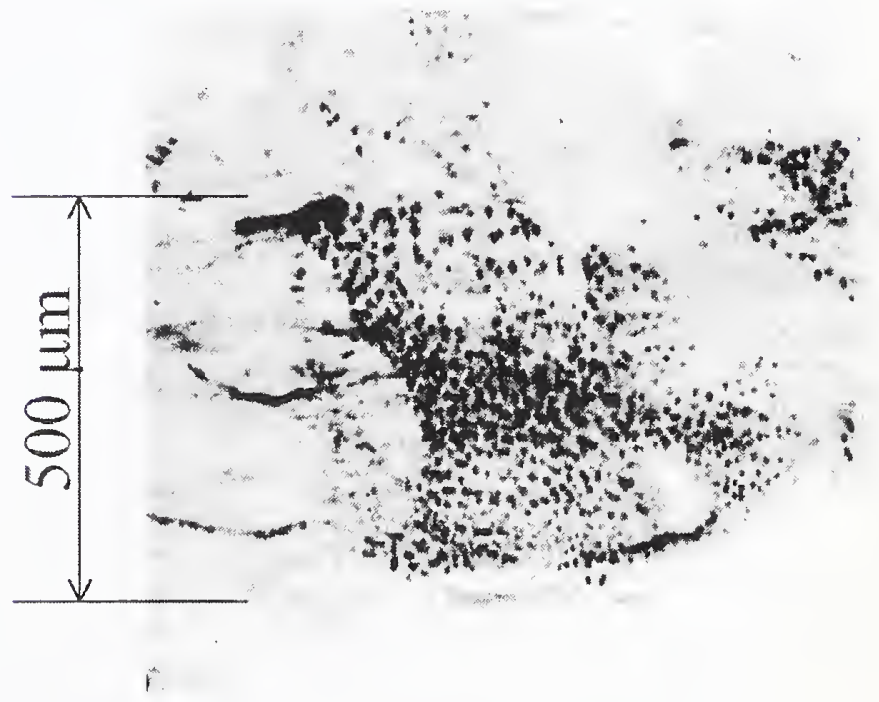

Fig 2: USAXS image of creep cavities in copper.

Fig. 1: USAXS of textured iron titanate. 



\section{Other}

Several important projects in the Ceramics Division are unique and are not constituent parts of "Programs." These projects are highly visible and have significant broad impact in several application areas but generally involve a commitment of personnel and resources less than that considered to be a program. The Ceramics Division relies on these projects for enhanced technology transfer and for more effective delivery of the results of the research programs to the technical community. 
Chemical Standards for Optoelectronic Semiconductors

\author{
Lawrence H. Robins, Albert J. Paul
}

Optoelectronic device makers require accurate data on the chemical composition of III-V semiconductor alloys, such as $A l_{x} G a_{1-x} A s$, to make their manufacturing processes more predictable and robust and to enable more accurate modeling and simulation of device performance. As part of a larger NIST project to develop standard reference materials to meet this need, we are improving the accuracy of room-temperature photoluminescence spectroscopy as an indirect, standards-based composition measurement method.
The objective of this project is to improve the accuracy of room-temperature photoluminescence (PL) spectroscopy as a chemical composition measurement method for III-V compound semiconductor films, focusing on the important $\mathrm{Al}_{\mathrm{x}} \mathrm{Ga}_{1-\mathrm{x}} \mathrm{As}$ and $\mathrm{In}_{\mathrm{x}} \mathrm{Ga}_{1-\mathrm{x}} \mathrm{As}_{\mathrm{y}} \mathrm{P}_{1-\mathrm{y}}$ systems. PL is widely used by the semiconductor materials and device industries as a composition metrology tool because of its speed, simplicity, and low cost. However, to achieve the high accuracy needed for semiconductor device simulation and modeling, the PL composition measurements must be calibrated using standard samples of accurately known composition. Heretofore, such samples have not been available.

The Compound Semiconductor Composition Standards Program at NIST is aimed at the development of high-accuracy composition standards for III-V compound semiconductor films. Thus far, our efforts have focused on the $\mathrm{Al}_{\mathrm{x}} \mathrm{Ga}_{1-\mathrm{x}} \mathrm{As}$ system. Films are grown in two molecular beam epitaxy (MBE) deposition systems and the compositions (parameter $x$ ) are measured both by in-situ monitoring, primarily reflection high-energy electron diffraction (RHEED), and by ex-situ chemical measurements, primarily wavelength dispersive $\mathrm{X}$-ray spectroscopy in an electron probe microanalyzer (WDS/EPMA).

The PL-based composition measurements rely on the linear or nearly linear composition $(x)$ dependence of the energy at the peak of the PL emission spectrum, which is closely tied to the semiconductor band gap. Careful data reduction and analysis techniques, including near-real-time calibration of the wavelength (or photon energy) scale with atomic vapor lamps, correction for the wavelength dependence of the spectrometer response function, and curve-fitting of the emission spectrum lineshape, enable us to determine the peak $\mathrm{PL}$ emission energy, denoted $\mathrm{E}_{\mathrm{PL}}$, with high accuracy and reproducibility. A platinum resistive temperature sensor is used to measure and correct for the effect of room temperature drift on $\mathrm{E}_{\mathrm{PL}}$. With these procedures, the reproducibility of $\mathrm{E}_{\mathrm{PL}}$ is found to be $\pm 0.0003 \mathrm{eV}$ or better (note all uncertainties are reported at the $2 \sigma$ level).

Measurements of $E_{P L}$ were made for a number of samples with compositions $(x)$ previously measured by the in-situ RHEED and ex-situ WDS methods. Calibration curves were generated by fitting a linear function to the $\left(\mathrm{E}_{\mathrm{PL}}, x\right)$ data. The best-fit line determined from the WDS compositions is

$$
x=\left(0.721 \pm 0.006 \mathrm{eV}^{-1}\right)\left[\mathrm{E}_{\mathrm{PL}}-(1.4232 \pm 0.0003) \mathrm{eV}\right]
$$

The deviations between the fitted PL compositions and the measured WDS compositions, as well as the corresponding deviations for PL vs. RHEED, are plotted in Fig 1. The deviations between the fitted line and the measured compositions are seen to be larger for $\left(\mathrm{E}_{\mathrm{PL}}, x_{\mathrm{RHEED}}\right)$ than for $\left(\mathrm{E}_{\mathrm{PL}}, x_{\mathrm{WDS}}\right)$. This result is plausible because the RHEED measurements are performed at the start of each deposition, and do not allow for possible composition drift during growth, while both the PL and WDS/EPMA measurements probe the upper portions of the as-grown films.

We will also examine heavily n-type or p-type doped samples, in which large doping shifts of $\mathrm{E}_{\mathrm{PL}}$ occur. The objective will be to develop an algorithm to determine $x$ from $\mathrm{E}_{\mathrm{PL}}$ and the dopant (or carrier) concentration.

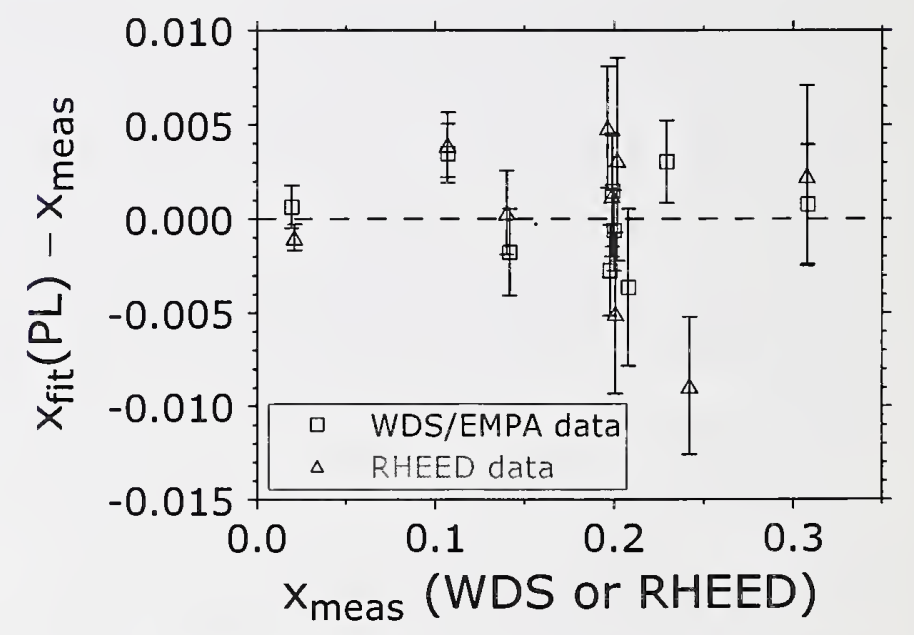

Fig. 1. Deviations of fitted (PL) from measured (WDS, RHEED) compositions of $\mathrm{Al}_{\mathrm{x}} \mathrm{Ga}_{1-\mathrm{x}} \mathrm{As}$ films. Uncertainties of the fitted PL compositions are derived from the uncertainties of the slope and the $\mathrm{E}_{\mathrm{PL}}$ values (as given in the equation). The uncertainties of the WDS compositions are estimated to be $\approx 0.002$; the uncertainties of the RHEED compositions are estimated to be $\leq 0.004$. 


\section{Composition, Structure and Optical Properties of Group III Nitride Films}

\author{
Lawrence H. Robins
}

Photonic device manufacturers need to better understand how phase separation and other types of compositional inhomogeneity affect the properties of the $I n_{x} G a_{1-x} N$ active layers of III-nitride visible light-emitting devices. We are addressing this issue by a systematic study of the composition (x) dependence of the structural and optical properties of relatively thick, bulk-like $I n_{x} G a_{1-x} N$ films grown by MOCVD on GaN/sapphire substrates.
We are measuring critical optical and structural properties of III nitride thin films ( $\mathrm{GaN}, \mathrm{AIN}, \mathrm{InN}$ and their alloys). The III nitrides are leading materials for short-wavelength solid-state optical emitters and detectors, and also show promise for high-power, high-frequency microelectronics. Our current objective is to better understand how compositional inhomogeneity affects the optical properties of indium gallium nitride $\left(\operatorname{In}_{\mathrm{x}} \mathrm{Ga}_{1-\mathrm{x}} \mathrm{N}\right)$ alloys, used to make the active layers of III nitride based visible-wavelength emitters. Some possible types of compositional inhomogeneity are In atom clustering, InN quantum dot formation, spinodal decomposition, and long-range ordering.

We examined $\operatorname{In}_{x} \mathrm{Ga}_{1-\mathrm{x}} \mathrm{N}$ films, grown by metalorganic chemical vapor deposition on $\mathrm{GaN} /$ sapphire substrates, with alloy compositions $(x)$ in the range $0.04<x<0.47$ and film thicknesses greater than $0.4 \mu \mathrm{m}$.

The average $\mathrm{In} /(\mathrm{In}+\mathrm{Ga})$ ratio within each $\operatorname{In}_{\mathrm{x}} \mathrm{Ga}_{1-\mathrm{x}} \mathrm{N}$ film, denoted $x_{\text {avg }}$, was determined by wavelength dispersive $\mathrm{x}$-ray spectroscopy in an electron probe microanalyzer (WDS/EPMA). Correlation of the $0006 \mathrm{x}$-ray diffraction (XRD) peaks in the films with the WDS results shows that the $c$ lattice constant is a linear function of $x_{\text {avg }}$ from $x=0$ to $x=1$ (Vegard's law). Analysis of indium K-edge EXAFS data shows that the $\mathrm{In} /(\mathrm{In}+\mathrm{Ga})$ ratio in the indium second shell, denoted $i_{\mathrm{N}=2}$, is equal to $x_{\text {avg }}$ within the measurement uncertainty. These results indicate that the films have structures close to random alloys.

Optical properties of the films were characterized by optical transmittance and cathodoluminescence (CL) spectroscopies. The electronic band gap energy $\left(\mathrm{E}_{\mathrm{G}}\right)$ of each film was estimated from the position of the absorption edge in the optical transmittance spectrum and, independently, from the highest energy peak in the CL spectrum. These two measures of $\mathrm{E}_{\mathrm{G}}$ correlate closely, as shown in Fig. 1. The dependence of $\mathrm{E}_{\mathrm{G}}$ on $x_{\mathrm{avg}}$ is described well by a quadratic function with a second-order parameter equal to $-4.57 \pm 0.75 \mathrm{eV}(2 \sigma$ uncertainty level, determined from the scatter between the data points and the quadratic fit). The physical mechanism for the large band gap bowing with composition is not yet understood.
The magnitude of the compositional fluctuations in the films, $\delta x$, was estimated by modeling the full width at half maximum (FWHM) of the 0006 XRD peak and of the band-edge CL peak. Let $\delta c$ be the XRD FWHM in lattice constant dimensions. If the variation of $c$ arises primarily from compositional fluctuations, then, from Vegard's law,

$$
\delta x_{\mathrm{XRD}}=\delta c /\left(c_{\mathrm{InN}}-c_{\mathrm{GaN}}\right)
$$

Similarly, let $\delta \mathrm{E}_{\mathrm{CL}}$ be the FWHM of the band-edge CL peak. If the variation of $\mathrm{E}_{\mathrm{CL}}$ arises primarily from band gap fluctuations that are correlated with composition, then

$$
\delta x_{\mathrm{CL}}=\delta \mathrm{E}_{\mathrm{CL}} /\left(\mathrm{dE}_{\mathrm{G}} / \mathrm{d} x\right)
$$

(where $\mathrm{dE}_{\mathrm{G}} / \mathrm{d} x$ is the slope of the quadratic curve shown in Fig. 1). The values of $\delta x$ obtained from the two model calculations are found to be in good agreement with each other and to lie near the line $\delta x=0.2 x_{\mathrm{avg}}$. This agreement supports the validity of both model calculations, because it is unlikely that two incorrect models would agree by chance.

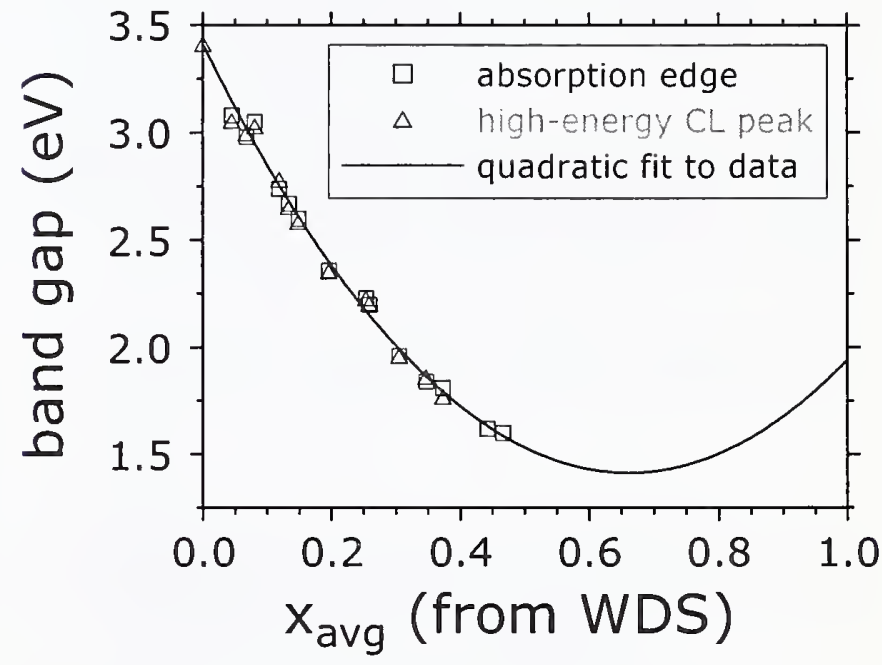

Fig. 1. Composition dependence of electronic band gap of $\mathrm{In}_{\mathrm{x}} \mathrm{Ga}_{1-\mathrm{x}} \mathrm{N}$ films, as measured by optical transmittance and cathodoluminescence (CL) spectroscopies. Measurement uncertainties of $x_{\text {avg }}$ are $\approx 0.001$; measurement uncertainties of the transmittance and CL band gaps are $0.03 \mathrm{eV}$.
Charles E. Bouldin, Igor Levin, Mark D. Vaudin, and Joseph C. Woicik, Ceramics

Division

John T. Armstrong, Chemical Science and Technology Laboratory

C.A. Parker, J.C. Roberts, and S.M. Bedair, Dept. of Electrical and Computer

Engineering, North Carolina State University 


\section{Phase Relationships in High Temperature Superconductors}

\author{
Winnie Wong-Ng, Lawrence P. Cook
}

Phase diagrams are central to the successful processing of high $T_{c}$ superconductor materials as conductors for power transmission. Of current interest for rapid advancement of second-generation coated conductor technology are the $\mathrm{RBa}{ }_{2} \mathrm{Cu}_{3} \mathrm{O}_{6+x}-t y p e$ superconductors. Issues addressed by current studies include the mixing of smaller and larger $R$ ions to optimize flux-pinning and melting properties, determination of phase relations in the $\mathrm{Ba}-\mathrm{Nd}-\mathrm{Cu}$-O system under conditions similar to industrial processing, and the phase evolution of $\mathrm{YBa}_{2} \mathrm{Cu}_{3} \mathrm{O}_{6+x}$ from barium-fluoride containing precursors.
Two parallel technologies are currently being pursued for the development of practical bulk conductors using cuprate hightemperature superconductors. The powder-in-tube (PIT) process, in which silver tubing is filled with superconductor powder and repeatedly rolled and heated, is the preferred method of fabrication for $\mathrm{Bi}-\mathrm{Pb}-\mathrm{Sr}-\mathrm{Ca}-\mathrm{Cu}-\mathrm{O}$ (BSCCO)-based superconductors. We have recently determined the primary phase fields for the principal superconducting phases in the multi-component BSCCO system. The rolling assisted by biaxially textured substrate/ion beam assisted deposition (RABiTS/IBAD) coated-conductor technology, in which the superconductor phase is used as a coating on a thin metallic ribbon substrate, is of interest primarily for fabrication of $\mathrm{RBa}_{2} \mathrm{Cu}_{3} \mathrm{O}_{7}$-type ( $\mathrm{R}=$ lanthanide and yttrium) superconductors. Current phase equilibria research is focused on understanding the dependence of the $\mathrm{BaO}-\mathrm{R}_{2} \mathrm{O}_{3}$ $\mathrm{CuO}_{\mathrm{x}}$ subsolidus relations on both oxygen partial pressure and choice of lanthanides. One of the most promising methods for producing high-quality superconductor is the ex situ method using e-beam co-evaporated Ba-F-Y-Cu precursor films on RABiTS, followed by a post-annealing in the presence of $\mathrm{H}_{2} \mathrm{O}$ vapor. The details of phase evolution of the superconducting phase during this process, however, are not understood. It is important to determine the role of phase equilibria, specifically, whether an intermediate fluorinecontaining low-temperature liquid forms, and, whether its formation plays a role in governing the formation of the $\mathrm{Y} 123$ phase. The investigation of the system $\mathrm{BaF}_{2}-\mathrm{BaO}-$ $\mathrm{Y}_{2} \mathrm{O}_{3}-\mathrm{CuO}_{\mathrm{x}}-\mathrm{H}_{2} \mathrm{O}$ is a second major thrust of our phase equilibria project.

To match the processing conditions of the RABiT/IBAD coated-conductors, we have established an experimental procedure for minimizing the presence of $\mathrm{CO}_{2}$ by using carbonate-free $\mathrm{BaO}$. The phase diagrams of the $\mathrm{Ba}-\mathrm{Nd}-\mathrm{Cu}-\mathrm{O}$ system under purified air $\left(900^{\circ} \mathrm{C}-930^{\circ} \mathrm{C}\right)$ and under $0.1 \%$ $\mathrm{O}_{2}\left(810^{\circ} \mathrm{C}\right)$ were completed. Detailed study of the subsolidus diagrams also included the characterization of the solid solution series $\mathrm{Ba}_{2-x} \mathrm{Nd}_{1+x} \mathrm{Cu}_{3} \mathrm{O}_{6+z}$. A comparison of the $\mathrm{Ba}-\mathrm{Nd}-\mathrm{Cu}-\mathrm{O}$ phase diagrams prepared under different conditions was also made.
By mixing $\mathrm{Yb}$ with the Nd123 solid solution, both melting and flux-pinning properties can be tailored and optimized. The solid solution range of $(\mathrm{Nd}, \mathrm{Yb})_{1+x} \mathrm{Ba}_{2-x} \mathrm{Cu}_{3} \mathrm{O}_{2}$ under pure air, $2 \% \mathrm{O}_{2}$ and $0.1 \% \mathrm{O}_{2}$ was shown to decrease as the oxygen partial pressure decreased. Characterization of the single phase region was conducted using neutron and X-ray diffraction. Melting temperatures were determined using DTA. Onset Tc values were obtained using AC magnetic susceptibility and SQUID measurements.

Our Mettler thermobalance system has been modified to allow different partial pressures and flow rates of water to react with samples in the $\mathrm{Ba}-\mathrm{Y}-\mathrm{Cu}-\mathrm{F}-\mathrm{O}$ system and subsystems. DTA/TGA studies of the eutectic melt composition in the $\mathrm{BaO}-\mathrm{Y}_{2} \mathrm{O}_{3}-\mathrm{CuO}$ system were conducted at various oxygen pressure under three processing conditions: (1) in the presence of water vapor, (2) with the addition of $\mathrm{BaF}_{2}$, and (3) with the presence of both $\mathrm{H}_{2} \mathrm{O}$ and $\mathrm{BaF}_{2}$. The partial pressure and flow rate of water were found to be critical in these experiments for the formation of the Y213 phase.

Results of our research have been reported at three international conferences in FY2000, and will be discussed during two invited lectures. The importance of this work is also acknowledged by the Department of Energy which has provided continuous partial support. By providing the phase equilibria data as the basis for optimal processing, high $T_{c}$ technology will be advanced through reductions in cost and improvements in performance. It is anticipated that high $T_{c}$ products in areas such as power transmission, motors, magnets, and energy storage will have a significant impact on the marketplace. High $\mathrm{T}_{\mathrm{c}}$-based power technologies are widely regarded as environmentally friendly as well as potentially cost effective due to their increased efficiency.
Contributors and Collaborators
I. Levin (Ceramics Division), Q. Huang, B. Toby (NIST Center for Neutron

Research), L. Swartzendruber (Metallurgy Division)

A. Kearsley (Mathematical and Computational Sciences Division)

T. Holesinger (LANL), R. Feenstra (ORNL), G. Riley (ASC), J. Kaduk (BP-

Amoco), R. Meng (U. Houston), D. Shaw (NYU), J. Suh, R. Coutts,

J. Dillingham (U. Maryland) 


\section{Ceramics Division Publications}

Allen, A.J., Fuller, E.R., Faber, K.T., Zimmerman, M.H., Wallace, J., "Anisotropic Small-Angle Scattering Studies of Ceramics," in'Advanced Ceramics for the $21^{\text {st }}$ Century: Proc. TMS 1999 Julia R. Weertman Symp.,' ed. Y-W. Chung, D.C. Dunard,P.K. Liaw, G.B. Olson, TMS, Warrendale, PA, 15 (1999).

Allen, A.J., Long, G.G., Jemian, P.R., Zschack, P., Ilavsky, J., "Ultrasmall-Angle X-ray Scattering Studies at a Third Generation Synchrotron Source," J. Appl. Cryst., in preparation.

Allen, A.J., Ilavsky, J., Long, G.G., Wallace, J., Berndt, C.C., Herman, H., "Microstructural Characterization of YttriaStabilized Zirconia Plasma-Sprayed Deposits Using Multiple Small-Angle Neutron Scattering," Acta. Mater., in press.

Allen, A.J., Livingston, R.A., "The Relationship Between Differences in Silica Fume Additives and the Fine Scale Microstructural Evolution in ement-Based Materials," Adv. Cem.-Based Mater., 8, 118 (1998).

Bar-On, I., Quinn, G. D., Salem, J. , and Jenkins, M. J., Fracture Toughness Standard Test Method C 1421-9 for advanced Ceramics," presented at the $32^{\text {nd }}$ National Symposium on Fatigue and Fracture, STM, June 16,2000, to be publ. ASTM STP 1406, 2001.

Allen, A.J., Thomas, J.J., Jennings, H.M., "Small-Angle Scattering Measurements of the C-S-H Gel Phase in Cements," J. Non-Cryst. Mater., in preparation.

Begley, E.F., Sturrock, C.P., MatML: XML for Materials Property Data, Advanced Materials and Processes, 158, (5) 72, 2000.

Black, D. , Polvani, R., and Quinn, G. D., "Using X-ray Topography to Study Fracture of Single-Crystal Ceramics," presented at the $4^{\text {th }}$ International Conference on the Fractography of Glasses and Ceramics, Alfred University, July 2000, to be publ., Ceramic Transactions, eds, J. Varner, G. Quinn, V. Frechette, American Ceramic Society, Westerville, $\mathrm{OH}, 2001$.

Blendell, J.,Vaudin, M., Fuller, E. J., Jr., "Determination of Texture Function from Individual Grain Orientation Measurements," J. Amer. Ceram. Soc., 82, (11) 3217 (1999).

Boukari, H., Allen, A.J., Long, G.G., Ilavsky, J., Wallace, J., Berndt, C.C., Herman, H., "Small-Angle Scattering Studies of the Role of Feedstock Particle Size on the Microstructural Behavior of Plasma-Sprayed YSZ
Deposits During Annealing," J. Am. Ceram. Soc., in preparation.

Burton, B.P. and Cockayne, E., "Why $\mathrm{Pb}\left(\mathrm{B}, \mathrm{B}^{\prime}\right) \mathrm{O}_{3}$ perovskites disorder at lower temperatures than $\mathrm{Ba}\left(\mathrm{B}, \mathrm{B}^{\prime}\right) \mathrm{O}_{3}$ perovskites," Phys. Rev. B 60 R12542 (1999).

Burton, B.P., "Why $\mathrm{Pb}\left(\mathrm{B}, \mathrm{B}^{\prime}\right) \mathrm{O}_{3}$ Perovskites Disorder More Easily than $\mathrm{Ba}\left(\mathrm{B}, \mathrm{B}^{\prime}\right) \mathrm{O}_{3}$ Perovskites, and the Thermodynamics of 1:1-type Short Range Order in PMN," J. Phys. Chem. Solids 61, 327 (2000).

Burton, B.P., "Long-range versus Short-range Interactions and the Configurational Energies of $\mathrm{Ba}\left(\mathrm{B}, \mathrm{B}^{\prime}\right) \mathrm{O} 3$ Perovskites," Modelling and Simulation in Materials Science and Engineering, 8, 211 (2000).

Bulter, E. P. and Fuller, Jr., E. R., "Composite Materials (Ceramic-Matrix)," in Kirk-Othmer Concise Encyclopedia of Chemical Technology, Fourth Edition, (John Wiley \& Sons, Inc., New York, NY, 1999), pp. 515-519.

Burton, B.P., "Order-Disorder and Phase Separation," for publication as a chapter in the Encyclopedia of Materials: Science and Technology, (Elsivier), L.E. Bennett Ed., WERB July 2000.

Chan, J. Y. Levin I., Vanderah T.A., Geyer R.G., and Roth, R.S. "Subsolidus Phase Relations and Dielectric Properties in the $\mathrm{SrO}-\mathrm{Al}_{2} \mathrm{O}_{3}-\mathrm{Nb}_{2} \mathrm{O}_{5}$ System," Int'l. J. Inorg. Mats. 2(1), 107-114 (2000).

Chen, C. I., Hsu, S. M., Kelley, F., "A Predictive Chemical Kinetics Model for Lubricant Performance in Diesel Engines," submitted to Tribology Trans.

Chuang, T.-J. and Fuller, Jr., E. R., "Analysis of Residual Stress State in Thermal Barrier Coatings." in Fracture Mechanics of Ceramics, Vol. 13, Eds. by R.C. Bradt, K. W. White, D. Munz, M. Sakai, and V. Ya. Shevchenko, (Plenum Press, New York, NY, 2001).

Cockayne, E. and Rabe, K.M., "Pressure Dependence of Instabilities in Perovskite $\mathrm{PbZrO}_{3}$," J. Phys. Chem. Solids 61, 305 (2000).

Cockayne, E. and Burton, B.P. "Phonons and Static Dielectric Constant in $\mathrm{CaTiO}_{3}$ from First Principles," Phys. Rev. B 62, 3735 (2000).

Cockayne, E., "Generation of Quasicrystals via a Single Cluster,” Mater. Sci. Engin. A (in press). 
Cockayne, E., "Comparative Dielectric Response in $\mathrm{CaTiO}_{3}$ and $\mathrm{CaAl}_{1 / 2} \mathrm{Nb}_{1 / 2} \mathrm{O}_{3}$ from First Principles," to be submitted to J. Appl. Phys.

Cook, L.P., Wong-Ng, W., "Vapor pressure of the $\mathrm{SrPbO}_{3}$ perovskite phase", Pervoskite Oxides for Electronic Energy Conversion and Energy Efficiency Applications, Ceramic Transactions 104, Eds. W. Wong-Ng, T. Holesinger, G.N. Riley and R. Guo, Published by ACerS, Westerville, OH 43086, pp. 61-69 (2000).

Cook, L.P., and Wong-Ng, W., "Phase equilibria of Ag with (Bi,Pb)-Sr-Ca-Cu-O (BSCCO) oxides and with $\mathrm{Pb}-2223$ under various oxygen pressures," Pervoskite Oxides for Electronic Energy Conversion and Energy Efficiency Applications, Ceramic Transactions 104, Eds. W. WongNg, T. Holesinger, G.N. Riley and R. Guo, Published by ACerS, Westerville, OH 43086, pp. 87-95 (2000).

Deckman, D., Chen, C. I., Hsu, S. M., "Effect of Selected Chemical Compounds on the Lubrication of Silicon Carbide," Tribology Trans, 42, 3, (1999).

Dieng, L.M., Tyson, T.A., Croft, M., Dogan, F., Kim, C.-Y., Woicik, J.C., and Grow, J., "Observation of changes in the atomic and electronic structure of single-crystal YBCO accompanying bromination: Understanding the recovery of superconductivity", submitted for publication, Appl. ++ Phys. Lett.

Dillingham, J., Wong-Ng, W., Levin, I., "Phase Equilibria of the $\mathrm{SrO}-\mathrm{Yb}_{2} \mathrm{O}_{3}-\mathrm{CuO}_{\mathrm{x}}$ System," submitted to Int. J. Inorg. Mater.

Dong, X., Yin, L., Jahanmir, S., and Ives, L. K., "Abrasive Machining of Glass-Ceramics with a Dental Handpiece," Machining Science and Technology, 4 (2000) 209-233.

Gates, R. S., Ying, T. N., Wang, Y. S., Yin, F., Chen, C. I., Hsu, S. M., "Mechanisms of Alcohols in Assisting the Diamond Grinding of Ceramics," Tribology Trans., 42, 3, (1999).

Gates, R. S., Hsu, S. M., "Chemo-mechanical Machining of Ceramics," Ceramic Trans., 102, 67-76 (1999).

Gabelnick A.M., Capitano A.T., Kane S.M., Gland J.L., Fischer D.A., "Propylene Oxidation Mechanisms and Intermediates Using In-Situ Soft X-ray Fluorescence Methods on the Pt(111) Surface", J. of Phy. Chem., 122(1), 143-149 (2000).

Gabelnick A.M., Burnett D.J., Gland J.L., Fischer D.A., "Molecular Mechanisms of Propyne Oxidation on the Pt(111) Surface: In Situ Soft X-ray Studies in Pressures of Oxygen" J. of Phys. Chem. B, in press.

Gamble L., Long J.R., Stayton P.S., Fischer D.A., Sambasivan S., Castner D.G., "Orientation of Surface Bound Proteins Studied by Near Edge X-ray Absorption Fine Structure,"
Sixth World Biomaterials Congress Transactions, 23(2000).

Genzer J., Sivaniah E., Kramer E.J., Wang J., Korner H., Char K., Ober C., DeKoven B., Bubeck R., Fischer D.A, Sambasivan S., "Temperature dependence of molecular orientation on the surfaces of semi-fluorinated polymers thin films", Langmuir, 16(4), 1993-1997 (2000).

Genzer J., Sivaniah E., Kramer E.J., Wang J., Korner H., Char K., Ober C., DeKoven B., Bubeck R., Chaudhury M., Sambasivan S., Fischer D.A, "The orientation of semifluorinated alkanes attached to polymers at the surface of polymer films", Macromolecules, 33(5), 1882-1887 (2000).

Genzer J., Sivaniah E., Kramer E.J., Wang J., Xiang M., Char K., Ober C.K., Bubeck R.A., Fischer D.A., Graupe M., Colorado R., Jr., Shmakova O.E., Lee T.R., "Molecular orientation of single and 2-armed monodendron semifluorinated chains on "soft" and "hard" surfaces studied using NEXAFS", Macromolecules, 33(16), 60686077 (2000).

Gupta, J.A., Watkins, S.P., Crozier, E.D., Woicik, J.C., Harrison, D.A., Jiang, D.T., Pickering, I.J., and Karlin, B.A., "Layer perfection in ultrathin InAs quantum wells in GaAs(001)", Phys. Rev. B 61, 2073 (2000).

Hackley, V.A., Guide to the Nomenclature of Particle Dispersion Technology for Ceramic Systems, NIST Special Publication 945, National Institute of Standards and Technology, Gaithersburg, MD (20899).

Haugan, T., Wong-Ng, W., Cook, L.P., Swartzendruber, L., Brown, H.J., Shaw, D.T., "Flux-Pinning of $\mathrm{Bi}_{2} \mathrm{Sr}_{2} \mathrm{CaCu}_{2} \mathrm{O}_{8+\mathrm{x}}$ and $\mathrm{Sr}_{2} \mathrm{CaAl}_{2} \mathrm{O}_{6}$ Defects," Pervoskite Oxides for Electronic Energy Conversion and Energy Efficiency Applications, Ceramic Transactions 104, Eds. W. Wong-Ng, T. Holesinger, G.N. Riley and R. Guo, Published by ACerS, Westerville, OH 43086, pp. 163175 (2000).

Haugan, T., Wong-Ng, W., Cook, L.P., Brow, H.J., Swartzendruber, L., Shaw, T.J., "Flux pinning of $\mathrm{Bi}_{2} \mathrm{Sr}_{2} \mathrm{CaCu}_{2} \mathrm{O}_{8+\mathrm{x}} / \mathrm{Ag}$ superconductors utilizing ( $\mathrm{Sr}, \mathrm{Ca})_{14} \mathrm{Cu}_{24} \mathrm{O}_{41}$, nanophase $\mathrm{Al}_{2} \mathrm{O}_{3}$ and $\mathrm{Au}$ particles," Physica C 335 (1-4) 129-133 (2000).

Hayakawa T., Wang J., Xiang M., Li X., Ueda M., Ober C.K., Genzer J., Sivaniah E., Kramer E.J., Fischer D.A., "The Effect of Changing End Groups on Surface Properties: Synthesis and Characterization of Poly(Styrene-bSemifluorinated Isoprene) Block Copolymers with $-\mathrm{CF}_{2} \mathrm{H}$ End Groups", Macromolecules, in press.

Hryniewicz, P., Szeri, A. Z., and Jahanmir, S., "Application of Lubrication Theory to Fluid Flow in Grinding. Part I: Flow Between Smooth Surfaces," Journal of Tribology, in press. 
Hryniewicz, P., Szeri, A. Z., and Jahanmir, S., "Application of Lubrication Theory to Fluid Flow in Grinding. Part II: Influence of Wheel and Workpiece Roughness," Journal of Tribology, in press.

Hryniewicz, P., Szeri, A. Z., and Jahanmir, S., "Coolant Flow in Surface Grinding with Non-Porous Wheels," International Journal of Mechanical Sciences, 42(2000) 2347-2367.

Hsu, S. M., Chen, C. I., "A new way to look at simulation by combining bench testing and a mathematical model," accepted for publication in ASTM STP 1404, 2000.

Hsu, S. M., Shen, M. C., "Wear Maps," invited chapter in the CRC Handbook of Modern Tribology, Editor in chief, Bharat Bhushan, to be published in 2001, 120 pages submitted.

Hsu, S. M., Gates, R. S., "Boundary Lubrication and boundary lubricating films," invited chapter in the CRC Handbook of Modern Tribology, editor in chief, Bharat Bhushan, to be published in 2001, 100 pages submitted.

Hsueh, C.-H. and Fuller, Jr., E. R., "Residual Stresses in Thermal Barrier Coatings: Effects of Interface Asperity Curvature/Height and Oxide Thickness," Materials Science and Engineering: A, 283 [1-2], 46-55 (2000).

Hsueh, C.-H. and Fuller, Jr., E. R., "Residual Stresses in Thermal Barrier Coatings: Analytical Modeling of Oxide Thickness Effects on Residual Stresses in Thermal Barrier Coatings," Scripta Materialia, 42 [8], 781-787 (2000).

Ilavsky, J., Long, G.G., Allen, A.J., Berndt, C.C., "Evolution of the Void Structure in Plasma-Sprayed YSZ Deposits During Annealing," Mater. Sci. Eng. A, 272, 215 (1999).

Jahanmir, S., Ramulu, M., and Koshy, P., (Ed.), Machining of Ceramics and Composites, Marcel Dekker, New York, NY (1999).

Jahanmir, S., Xu, H. K., and Ives, L. K., "Mechanisms of Material Removal in Abrasive Machining of Ceramics," $\mathrm{S}$. Jahanmir, M. Ramulu, and P. Koshy (Ed.), Machining of Ceramics and Composites, Marcel Dekker, New York, NY (1999) 11-84.

Jenkins, M., Quinn, G. D., Salem, J. A., Bar-on, I., "Development, Verification and Implementation of a National Full-Consensus Fracture Toughness Test Method Standard for Advanced Ceramics," to be publ. in Fracture Resistance Testing of Monolithic and Composite Brittle Materials, ASTM STP 1409, J. A. Salem, M. G. Jenkins, and G. D. Quinn, eds., ASTM, West Conshohocken, PA 2001.

Jensen, R.P., Luecke, W.E., Padture, N.P., Wiederhorn, S.M., "High-Temperature Properties of Liquid-Phase Sintered $\alpha$-SiC", Mater. Sci. Eng. A, A282, 109-114 (2000).
Jillavenkatesa, A., Dapkunas, S.J., Lum, L.H., "NIST Recommended Practice Guide: Particle Size Characterization," NIST SP 960-1, National Institute of Standards and Technology, Gaithersburg, MD (20899).

Kaiser, D.L., Vaudin, M.D., Rotter, L.D., Bonevich, J.E., Levin, I., Armstrong, J.T., Roytburd, A.L., Schlom, D.G., "Effect of Film Composition on the Orientation of $(\mathrm{Ba}, \mathrm{Sr}) \mathrm{TiO}_{3}$ Grains in $(\mathrm{Ba}, \mathrm{Sr})_{y} \mathrm{TiO}_{2+y}$ Thin Films," J. Mater. Res. 14, 4657 (1999).

Kakisawa, H., Fuller, Jr., E. R., and Kagawa, Y., "Effect of Abrasive Wear on Interfacial Sliding Shear Resistance in $\mathrm{SiC}$ Fiber-Reinforced $\mathrm{Al}_{2} \mathrm{O}_{3}$ Matrix Composites," Acta Materialia, in press.

Kanematsu, W., Sando, M., Ives, L., Marinenko, R., and Quinn, G., "Determination of Machining Crack Geometry by Dye Penetration Technique," accepted by Journal of the American Ceramic Society.

Kanematsu, W., Sando, M., Ives, L. K., Marienenko, R. and Quinn, G. D., "Dye Impregnation Method for Revealing Machining Crack Geometry," accepted to be published by J. Am. Ceram. Soc.

Kelly, J. F. and Pei, P., "Certification of Ceramic Particle Size Distribution Reference Materials at NIST," American Ceramic Bulletin, in press.

Kessler, H., Fuller, Jr., E. R., and Balke, H., "Modeling of Stable and Unstable Polarization Switching," proceedings of SPIE's 7th Annual International Symposium on Smart Structures and Materials, edited by Christopher Lynch, 59 March 2000, Newport Beach, California, (2000).

Koshy, P., Ives, L. K., and Jahanmir, S., "Simulation of Diamond Ground Surfaces," International Journal of Machine Tools and Manufacture, 39(1999) 1451-1470.

Krause, Jr., R .F., Weiderhorn, S.M., and Kuebler, J.J., "Misalignment Induced Bending in Pin-Loaded Tensile Creep Specimens," J. Am. Ceram. Soc. 84145 (2001)

Lee, T.-L., Pillai, M., Labanda, G., Woicik, J.C., Lyman, P.F., Barnett, S.A., and Bedzyk, M.J., "Atomic-resolution study of the lattice distortion of a buried InGaAs monolayer in GaAs(001)", Phys. Rev. B 60, 13612 (1999).

Levin I. Bendersky L.A., Cline J.P., Roth R.S., and Vanderah, T.A., "Octahedral Tilting and Cation Ordering in Perovskite-Like $\mathrm{Ca}_{4} \mathrm{Nb}_{2} \mathrm{O}_{9}=3 \mathrm{Ca}\left(\mathrm{Ca}_{1 / 3} \mathrm{Nb}_{2 / 3}\right) \mathrm{O}_{3}$ Polymorphs," J. Solid State Chem. 150, 43-61 (2000).

Levin, I., Leapman, R.D., Kaiser, D.L., Van Buskirk, P.C., Bilodeau, S., Carl, R., "Accommodation of Excess Ti in a $(\mathrm{Ba}, \mathrm{Sr}) \mathrm{TiO}_{3}$ Thin Film With $53.5 \%$ Ti Deposited on $\mathrm{Pt} / \mathrm{SiO}_{2} / \mathrm{Si}$ by MOCVD," Appl. Phys. Lett. 75, 1299 (1999). 
Levin, I., Leapman, R.D., Kaiser, D.L., "Microstructure and chemistry of non-stoichiometric $(\mathrm{Ba}, \mathrm{Sr}) \mathrm{TiO}_{3}$ thin films deposited by MOCVD,” J. Mater. Res. 15, 1433 (2000).

Levin, I., Robins, L.H., Vaudin, M.D., Tuchman, J.A., Lakin, E., Sherman, M.J., Ramer, J., "Spontaneous compositional modulation in the $\mathrm{AlGaN}$ layers of a thick $\mathrm{AlGaN} / \mathrm{GaN}$ multilayer structure," J. Appl. Phys., in press.

Lipaascu, D., Nuffer, J., Wallace, J. and Roedel, J. Role of Crack Formation in the Electric Fatigue Behavior of Ferroelectric Ceramics, of SPIE's 7th Annual International Symposium on Smart Structures and Materials, edited by Christopher Lynch, 5-9 March 2000, Newport Beach, California, (2000), in press.

Livingston, R.A., Neumann, D.A., Allen, A.J., FitzGerald, S., Berliner, R., "Application of Neutron Scattering to Portland Cement," Neutron News, in press.

Long, G.G., Allen, A.J., Ilavsky, J., Jemian, P.R., Zschack, P., "The Ultrasmall-Angle X-ray Scattering Instrument on UNICAT at the APS," in "Synchrotron Radiation Instrumentation: $11^{\text {th }}$ U.S. National Conf.,' ed. P. Pianetta et al., AIP, 183 (2000).

Marienko, R.B., Armstrong, J., Kaiser, D.L., Ritter, J.J., Schenck, P.K., Bouldin, C., Blendell, J.E., Levin, I., "Manufacture and Electron Microprobe Characterization of Barium-Strontium-Titanate (BST) Films," Proceedings of the 2000 International Conference on Characterization and Metrology of ULSI, 2000.

McGuiggan, P. M., Hsu, S. M., Fong, W., Bogy, D., and Bhatia, C. S., "Friction Measurements of Ultra-Thin Carbon Overcoats in Air," accepted ASME J. of Tribology.

McGuiggan, P. M., Zhang, J., Hsu, S. M., "Comparison of Friction Measurements using the Atomic force Microscope and the Surface Forces Apparatus: The Issue of Scale."

Meyer, K., Rohl-Kuhn, B., Minor, D. and Klobes, P., "Special Features Regarding the Certification of Highly Particulated and/or Porous Reference Materials," Symposium Reference Materials for Technologies in the New Millennium, European Federal of National Associations of Measurement, Testing and Analystical Laboratories, Berlin Germany, May 22-23, 2000.

Minor, D. B., "A Re-Examination of a Specific Surface Area Research Material," Symposium-Reference Materials for Technologies in the New Millennium, European Federal of National Associations of Measurement, Testing and Analystical Laboratories," Berlin, Germany, May 22-23, 2000.

Moodenbaugh, A.R., Nielsen B., Sambasivan S., Fischer D.A., "Hole State Density of $\mathrm{La}_{1-\mathrm{x}} \mathrm{Sr}_{\mathrm{x}} \mathrm{CoO}_{3-\delta}(0 \leq \mathrm{x} \leq 0.5)$ Across the Insulator/Metal Phase Boundary", T. Friessnegg, S.
Aggarwal, and R. Ramesh Phys. Rev., B61, 5666-5671 (2000).

Munro, R.G. and Freiman, S.W., "Correlation of Fracture Toughness and Strength," J. Am. Ceram. Soc. 82, 2246 (1999).

Munro, R.G., "Material Properties of Titanium Diboride," J. Res. Natl. Inst. Stand. Technol. 105, 709 (2000).

Nagarajan, V. S., Hockey, B. Jahanmir, S., and Thompson, V. P., "Contact Wear Mechanisms of a Dental Composite with a High Filler Content," J. Mat. Science, 35(2000) 487-496.

Nakao, H., Ohwada, K., Takesue, N., Fujii, Y., Isobe, M., Ueda, Y., Zimmermann, M.v., Hill, J.P., Gibbs, D., Woicik, J.C., Koyama, I., and Murakami, Y., "X-ray anomalous scattering study of charge-ordered state in $\mathrm{NaV}_{2} \mathrm{O}_{5}$ ", to be published, Phys. Rev. Lett.

Nelson, E.J., Woicik, J.C., Hong, M., Kwo, J., Mannaerts, J.P., "Extended x-ray absorption fine-structure measurement of bond-length strain in epitaxial $\mathrm{Gd}_{2} \mathrm{O}_{3}$ on $\mathrm{GaAs}(001)$ ", Appl. Phys. Lett. 76, 2526 (2000).

Paik, U. and Hackley, V.A., "Influence of Solids Concentration on the Isoelectric Point of Aqueous Barium Titanate", J. Am. Ceram. Soc 83, 2381 (2000).

Parker, C.A., Roberts, J.C., Bedair, S.M., Reed, M.J., Liu, S.X., El-Masry, N.A., Robins, L.H., "Optical Band Gap Dependence on Composition and Thickness of $\left.\mathrm{In}_{\mathrm{x}} \mathrm{Ga}_{1-\mathrm{x}} \mathrm{N}()<\mathrm{x}<0.25\right)$ Grown on GaN", Appl. Phys. Lett. 75, 2566 (1999).

Pei P., Bhardwaj M.C., Anderson J., Minor D., and Thornton, T., "Measuring Green Body Density", Pages 43-46, Ceramic Industry, July 2000.

Perettie, D. J., DeKoven, B. M, Morgan, T. A., Fischer, D. A., Hsu, S. M., Talke, F. E., Kang, H. J., Bhatia, C. S., "The Use of Advanced Lubricant Additives to Enhance the Performance of the Head/Disk Interface: The Effects of Surface Energy and Orientation," Lubrication Engineers, 55, 22-27

Potrepka D.M., Budnick J.I., Moodenbaugh A.R., Fischer D.A., Hines W.A., "Combined ${ }^{63,65} \mathrm{Cu}$ NQR and NMR study of $\mathrm{Y}_{1-\mathrm{x}} \mathrm{Ca}_{\mathrm{x}} \mathrm{Ba}_{2} \mathrm{Cu}_{3} \mathrm{O}_{\mathrm{y}}$ ", Physica $\mathrm{C}$, in press.

Quinn G.D., Ives L.K, Jahanmir S., and Koshy P., "Fractographic Analysis of Machining Cracks in Silicon Nitride Rods and Bars," presented at the 4th International Conference on the Fractography of Glasses and Ceramics, Alfred University, July 2000, to be publ., Ceramic Transactions, eds, J. Varner, G. Quinn, V. Frechette, American Ceramic Society, Westerville, OH, 2001. 
Quinn G.D. and Swab J., "Comparisons of Calculated and Measured Flaw Sizes," presented at the 4th International Conference on the Fractography of Glasses and Ceramics, Alfred University, July 2000, to be publ., Ceramic Transactions, eds, J. Varner, G. Quinn, V. Frechette, American Ceramic Society, Westerville, OH, 2001.

Quinn, G. D., Xu, K., and Gettings, R., Standard Reference Material 2100: Fracture Toughness of Ceramics," Ceram. Eng. and Sci. Proc., 4 [20] Issue 3, 513-523 (1999).

Quinn, G. and Swab, J., "Elastic Modulus by Resonance of Rectangular Prisms: Corrections for Chamfers," J. Am. Ceram. Soc., 83 [2] $317-320$ (2000). Also Publ. as U. S. Army ARL TN 165, July 2000.

Quinn, G. D., "Indentation Hardness Testing of Ceramics," pp $244-251$ in Materials Testing and Evaluation, Vol 8, Mechanical Testing, ASM, Materials Park, OH, 2000.

Quinn, G. D., "VAMAS After Twelve," Bul. Amer. Ceram. Soc., Vol. 78, [7], pp. 78-83 (1999).

Quinn, G. D., "The Fracture Toughness Round Robins in VAMAS: What We Have Learned," in Fracture Resistance Testing of Monolithic and Composite Brittle Materials, ASTM STP 1409, J. A. Salem, M. G. Jenkins, and G. D. Quinn, eds., ASTM, West Conshohocken, PA, 2001.

Quinn, G. D., Xu, K., Gettings. R. J., Salem, J. A., and Swab, J. J., "Does Anyone Know the Real Fracture Toughness? SRM 2100: the World's First Ceramic Fracture Toughness Reference Material," Fracture Resistance Testing of Monolithic and Composite Brittle Materials, ASTM STP 1409, J. A. Salem, M. G. Jenkins, and G. D. Quinn, eds., ASTM, West Conshohocken, PA, 2001.

Quinn, G., "Fracture Toughness Testing of Ceramics," Chapter 4 9-7d of Encyclopedia of Materials: Science and Technology," Elsevier, to be publ. 2001.

Quinn, G. D., Xu, K., Gettings, R., Salem, J. A., and Swab, J., "Standard Reference Material 2100: Ceramic Fracture Toughness," presented at the $32^{\text {nd }}$ National Symposium on Fatigue and Fracture, ASTM, June 16,2000, to be publ. ASTM STP 1406, 2001.

Quinn, G., "Special Mechanical Testing Methods for Ceramics," Chapter 4-9-7n in Encyclopedia of Materials Science and Technology," Elsevier, to be publ. 2001

Radovic, M., Barsoum, M.J., El-Raghy, T., Seidensticker, J., Wiederhorn, S.M. "Tensile Properties of $\mathrm{Ti}_{3} \mathrm{SiC}_{2}$ in the 25-1300C Temperature Range", Acta Materiala, 48 453459 (2000).

Ravikiran, A. and Jahanmir, S., "Effect of Interfacial Layers on Wear Behavior of a Dental Ceramic," Journal of the American Ceramic Society, 83 (2000) 831-1833.
Ravikiran, A. and Jahanmir, S., Effect of Contact Pressure and Load on Wear of Alumina," Wear, in press.

Salem, J., Ghosn, L., Jenkins, M., and Quinn, G. , "Stress Intensity Factor Coefficients for Chevron Notched Flexure Specimens and a Comparison of Fracture Toughness Methods," Ceram. Eng. and Sci. Proc., 20, Issue 3, 503-512 (1999).

Stalick, J., Ilavsky, J. and Wallace, J., Thermal-Spray Yttriastabilized Zirconia Phase Changes, Proceedings of the International Thermal Spray Conference 2000,

Robins, L.H., Armstrong, J.T., Marienko, R.B., Vaudin, M.D., Bouldin, C.P., Woicik, J.C., Paul, A.J., Thurber, W.R., Miyano, K.E., Parker, C.A., Roberts, J.C., Bedair, S.M., Piner, E.L., Reed, M.J., El-Masry, N.A., Donovan, S.M., Pearton, S.J., "Optical and structural studies of strainrelaxed $\mathrm{In}_{\mathrm{x}} \mathrm{Ga}_{1-\mathrm{x}} \mathrm{N}$ films on $\mathrm{GaN} / \mathrm{sapphire}$ with $0.04<x<0.47$," Phys. Rev. B., in press.

Robins, L.H., Lowney, J.R., Wickenden, D.K., "Cathodoluminescence, photoluminescence and optical absorbance spectroscopy of aluminum gallium nitride $\left(\mathrm{Al}_{x} \mathrm{Ga}_{1-\mathrm{x}} \mathrm{N}\right)$ films," J. Mater. Res. 13, 2480 (1998).

Robins, L.H., Wickenden, D.K., "Spatially resolved luminescence studies of defects and stress in aluminum gallium nitride films," Appl. Phys. Lett. 71, 3841 (1997).

Robins, L.H., Armstrong, J.T., Marinenko, R.B., Vaudin, M.D., Bouldin, C.E., Woicik, J.C., Paul, A.J., and Thurber, W.R., "Optical and structural studies of compositional inhomogeneity in strain-relaxed indium gallium nitride films", submitted for publication, Proceedings of ISCS-27, 27th International Symposium on Compound Semiconductors.

Robins, L.H., Armstrong, J.T., Vaudin, M.D., Bouldin, C.E., Woicik, J.C., Paul, A.J., Thurber, W.R., Miyano, K.E., Parker, C.A., Roberts, J.C., Bedair, S.M., Piner, E.L., Reed, M.J., El-Masry, N.A., Donovan, S.M., Pearton, S.J., "Optical and structural studies of strain-relaxed $\operatorname{In}_{\mathrm{X}} \mathrm{Ga}_{1-\mathrm{X}} \mathrm{N}$ films on $\mathrm{GaN} /$ saphire with $0.04<\mathrm{x}<0.47$ ", submitted for publication, Phys. Rev. B.

Rodriguez J.A., Jirask T., Dvorak J., Sambasivan S., Fischer D.A., "Reaction of $\mathrm{NO}_{2}$ with $\mathrm{Zn}$ and $\mathrm{ZnO}$ : Photoemission, XANES and Density Functional Studies of the Formation of $\mathrm{NO}_{3}$ ", J. of Phys. Chem., 104, 319-328(2000).

Rodriguez J.A., Jirask T., Sambasivan S., Fischer D.A., Maiti A., "Chemistry of $\mathrm{NO}_{2}$ on $\mathrm{CeO}_{2}$ and $\mathrm{MgO}$ : Experimental and Theorical Studies on the Formation of $\mathrm{NO}_{3}$ ", J. of Phys. Chem.,112, 9929-39 (2000). 
Salem, J., Ghosn, L., Jenkins, M., and Quinn, G. , "Stress Intensity Factor Coefficients for Chevron Notched Flexure Specimens and a Comparison of Fracture Toughness Methods," Ceram. Eng. and Sci. Proc., 20, Issue 3, 503-512 (1999).

Sambasivan S., Fischer D.A., Shen M., Tesk J., Hsu S.M., "Effects of Annealing on UHMWPE Molecular Orientation", Sixth World Biomaterials Congress Transactions, 178(2000).

Sambasivan S., Fischer D.A., Kuperman A., DeKoven B.M., Direct Observation of Propylene Transformation Chemistry on and in the Pores of Silver Exchanged Faujasite Catalyst", Advanced Materials, in press.

Seabaugh, M.M., Vaudin, M.D., Messing, G.L., "Texture Development and Microstructure Evolution in LiquidPhase Sintered $\alpha-\mathrm{Al}_{2} \mathrm{O}_{3}$ Ceramics Prepared by Templated Grain Growth,", J. Amer. Ceram. Soc, in press.

Shen, M. C., Hsu, S. M., "Modeling Wear of Ceramics based on Wear Maps," in The advancing frontier of engineering tribology, edited by Qian Wang, Jim Netzel, and Farshid Sadeghi, published in STLE Special Symposium Proceeding honoring Prof. H. S. Cheng of Northwestern University, Oct. 1999, pp.140-149.

Shen, M. C., Hsu, S. M., Tesk, J. A., Christou, A., "Wear Analysis of UHMWPE using a Load Sum Method," Biomaterials Transactions, May, 2000.

Smith, D.T., "Use of Instrumented Indentation to Determine Mechanical Properties of Thin Films, Coatings and Functionally Graded Materials," to appear in Proceedings of Functionally Graded Materials in the 21st Century: A Workshop on Trends and Forecasts, Ed: K. Ichikawa, Kluwer Academic (2000).

Stalick, J., Ilavsky, J. and Wallace, J., Thermal-Spray Yttriastabilized Zirconia Phase Changes, pp 1185-1189 in "Thermal Spray Surface Engineering via Applied Research," Proceedings of the $1^{\text {st }}$ Thermal Spray Conference, 8-11 May 2000, Montreal Quebec, Canada, C.C. Berndt (ed), ASM International, Materials Park, OH, USA (2000).

Tesk, J., Johnson, C. E., Skrtic, D., Tung, M. S., Hsu, S. M., "Amorphous Alloys Containing Cobalt for Orthopaedic Applications," ASTM STP 1365, Proceedings of ASTM Special Symposium 'Cobalt-Base Alloys for Biomedical Applications', Desigi, J. A., Kennedy, R. L., and Pilliar, R. Eds., pp. 32-46, American Society for Testing and materials, West Conshohocken, PA 1999.

Thomas, J.J., Jennings, H.M., Allen, A.J., "The Surface Area of Cement Paste as Measured by Neutron Scattering - Evidence for Two C-S-H Morphologies," Cem. Concr. Res., 28, 897 (1998).
Thomas, J.J., Jennings, H.M., Allen, A.J., "Determination of the Neutron Scattering Contrast of Hydrated Portland Cement Paste Using $\mathrm{H}_{2} \mathrm{O} / \mathrm{D}_{2} \mathrm{O}$ Exchange," Adv. Cem.Based Mater., 7, 119 (1998).

Thomas, J.J., Jennings, H.M., Allen, A.J., "The Surface Area of Hardened Cement Paste as Measured by Various Techniques," Concr. Sci. and Eng., 1, 45 (1999).

Vanderah T.A., Febo W., Chan J.Y., Roth R.S., Loezos M., Rotter L.D., Geyer R.G., and Minor D.B., "Phase Equilibria and Dielectric Behavior in the $\mathrm{CaO}-\mathrm{Al}_{2} \mathrm{O}_{3}$ $\mathrm{Nb}_{2} \mathrm{O}_{5}$ System," J. Solid State Chem. 154, xxx (2000).

Vaudin, M.D., Rupich, M.W., Jowett, M., Riley, G.N., Bingert, J.F., "A Method for Crystallographic Texture Investigations Using Standard X-ray Equipment”, J. Mater. Res., 13, 2910 (1998).

Vaudin, M.D., “Accurate Texture Measurements on Thin Films Using a Powder X-ray Diffractometer", Proceedings of the Twelfth International Conference on Textures of Materials, NRC Research Press, Ottawa, 186 (1999).

Vaudin, M.D., Fox, G.R., "Measuring bimodal crystallographic texture in ferroelectric $\mathrm{PbZr}_{\mathrm{x}} \mathrm{Ti}_{1-\mathrm{x}} \mathrm{O}_{3}$ thin films," Ferroelectric Thin Films VIII, MRS Proceedings, 2000, in press.

Vedula, V. R., Glass, S. J., Saylor, D. M., Rohrer, G. S., Carter, W. C., Langer, S. A., and Fuller, Jr., E. R., "Residual Stress Predictions in Polycrystalline Alumina," J. Am. Ceram. Soc., in press.

Wang, P.S. “Assessment of Moisture Measurement Techniques for Ceramic Processing," NISTIR 6543, August 2000.

Wang, Z., Jiang, Q., Blendell, J.E., White, G.S., “Atomic Force Microscopy Observations of Domains in Fine Grain PZT Ceramics," submitted to Ferroelectrics.

Wasche, R., Naito, M., and Jahanmir, S., "Characterization Methods for Ceramic Powders and Green Bodies," VAMAS Bulletin, (2000).

Wasche, R., Naito, M. and Hackley, V. A., "Experimental Study on Zeta Potential and Streaming Potential of Advanced Ceramic Powders," Powder Technology, in press.

Widom, M., Al-Lebyani I., Wang Y., and Cockayne E., "Energetics of transition metal ordering in Al-Cu-Co," Mater. Sci. Engin. A (in press).

Wittberg. T.N. and Wang P.S., "XPS Study of the Dehydration of Clay and Kaolin Powders," Surface and Interface Analysis, Vol. 27, 936-940 (1999). 
Woicik, J.C., Nelson, E.J., Kendelewicz, T., and Pianetta, P., "Site-specific valence-electronic structure by x-ray standing waves," submitted for publication, Proceedings of the 20th National Congress of the Mexican Vacuum Society and the III Workshop on Optoelectronic Materials and their Applications.

Woicik, J.C., Nelson, E.J., Kendelewicz, T. Pianetta, P., Jain, M., Kronik, L., and Chelikowsky, J.R., "Partial density of occupied valence states by x-ray standing waves and highresolution photoelectron spectroscopy," submitted for publication, Phys. Rev. B, Rapid Communications.

Woicik, J.C., Nelson, E.J., Pianetta, P., "Direct measurement of valence-charge asymmetry by x-ray standing waves," Phys. Rev. Lett. 84, 773 (2000).

Wong-Ng, W., Cook, L.P., Greenwood, W., Kearsley, A., "Effect of Ag on the Primary Phase Field of High $\mathrm{T}_{\mathrm{c}}$ (Bi,Pb) 2223 Superconductor," J. Mater. Res., 15 (2) 296305 (2000).

Wong-Ng, W., Cook, L.P., "Melting Equilibria of the 2223 ([Bi,Pb]-Sr-Ca-Cu) High Tc Superconductor in Oxygen," Pervoskite Oxides for Electronic Energy Conversion and Energy Efficiency Applications, Ceramic Transactions 104, Eds. W. Wong-Ng, T. Holesinger, G.N. Riley and R. Guo, Published by ACerS, Westerville, OH 43086, pp. 97-106 (2000).

Wong-Ng, W., Cook, L.P., Greenwood, W., Kearsey, A., Lawrence, C., "Primary Phase Field of the $\mathrm{Pb}$-doped 2223 High Tc Superconductor in the (Bi,Pb)-Sr-Ca-Cu-O System," J. Res. Natl. Inst. Stand. Technol., 104 277-289 (1999).

Wong-Ng, W., Dillingham, J., and Cook, L.P., "Phase Equilibria of the $\mathrm{SrO}-\mathrm{Ho}_{2} \mathrm{O}_{3}-\mathrm{CuO}_{x}$ System", J. Solid State Chem., 149 333-337 (2000).

Wong-Ng, W., Swartzendruber, L., Kaduk, J.A., Bennett, L.H., "Magnetic and Structural Properties of the 'Brown Phase' Solid Solution $\mathrm{Ba}\left(\mathrm{Nd}_{2-\mathrm{x}} \mathrm{La}_{\mathrm{x}}\right) \mathrm{CuO}_{5}$," submitted to J. Mag. and Mag. Mater.

Wong-Ng, W., Kaduk, J.A., Dillingham, J.,"Crystallographic studies and $x$-ray reference patterns of $\mathrm{Ba}_{5} \mathrm{R}_{8} \mathrm{Zn}_{4} \mathrm{O}_{21}$ by Rietveld Refinements," submitted to Powd. Diffr.

Wong-Ng, W., Kaduk, J.A., Huang, Q., Roth, R.S., "Crystal Structure of Monoclinic Perovskite $\mathrm{Sr}_{3.94} \mathrm{Ca}_{1.31} \mathrm{Bi}_{2.7} \mathrm{O}_{12,}$," Powd. Diffr., in press.

Woo, H. Tyson, T.A., Croft, M., Cheong, S.-W., Woicik, J.C., and Brandt, B.L., "Correlationis between the magnetic and structural properties of $\mathrm{Ca}$ doped $\mathrm{BiMnO}_{3}$ ", submitted for publication, Phys. Rev. B.

Wooley, P. H., Z. Song, L. Mayton, S. Nasser, U. Cho, M. C. Shen, S. M. Hsu, "Variations in UHMWPE particle morphology result in different cellular and Cytokine
Responses in the Murine Air Pouch Model," Trans. of Biomaterials Society, May, 2000.

Xiang M., Li X., Char K., Ober C.K., Genzer J., Sivaniah E., Kramer E.J., Fischer D.A., "Surface Stablity in LiquidCrystalline Block Copolymers with Semifluorinated Monodendron Side Groups," Macromolecules, 33(16), 6106-6119 (2000).

Yin, L., Ives, L. K., and Jahanmir, S., "Machining Behavior of Glass-Infiltrated Alumina," Machining Science and Technology, in press.

Yoon, K.J., Wiederhorn, S.M., Luecke, W.E., "Comparison of Tensile and Compressive Creep Behavior in Silicon Nitride," J. Am. Ceram. Soc. 83[8] 2017-2022 (2000).

Zhang, X. H., Gates, R. S., Anders, S., Hsu, S. M., “An Accelerated Wear Test Method to Evaluate Lubricant thin film on Magnetic Hard Disks," submitted to Tribology Letters.

Zimmermann, A., Fuller, Jr., E. R., and Carter, W. C., "Damage Evolution During Microcrack Formation in Brittle Solids," Acta Materialia, in press.

Zimmermann, A, Fuller, Jr., E. R., and Rödel, J., "Residual Stress Distributions in Ceramics," J. Am. Ceram. Soc., Vol. 82, No. 11, 3155-3160 (1999). 


\section{Ceramics Division}

\section{Chief}

Stephen W. Freiman

Phone: 301-975-6119

E-mail: stephen.freiman@nist.gov

\section{Deputy Chief}

S. J. Dapkunas

Phone: 301-975-6119

E-mail: stanley.dapkunas@nist.gov

\section{Group Leaders}

Ceramic Manufacturing

Said Jahanmir

Phone: 301-975-3671

E-mail: said.jahanmir@nist.gov

Phase Equilibria

Terrell Vanderah

Phone: 301-975-5785

E-mail: terrell.vanderah@nist.gov

Film Characterization and Properties Debra Kaiser

Phone: 301-975-6759

E-mail: debra.kaiser@nist.gov

Materials Microstructural Characterization

Gabrielle Long

Phone: 301-975-5975

E-mail: gabrielle.longi@nist.gov

Surface Properties

Stephen Hsu

Phone: 301-975-6120

E-mail: stephen.hsu@nist.gov

Data Technologies

S. J. Dapkunas

Phone: 301-975-6119

E-mail: stanley.dapkunas@ nist.gov 


\section{Research Staff}

Allen, Andrew

andrew.allen@nist.gov

Small angle $x$-ray scattering

Ceramic microstructure analysis

Begley, Edwin

edwin.begley@nist.gov

Materials informatics

Database management methods

Engineering database structures

Belsky, Alec

alec.belsky@nist.gov

Crystallographic database

Black, David

david.black@nist.gov

Defect microstructures

Polycrystalline diffraction imaging

$\mathrm{X}$-ray imaging

Blendell, John

john.blendell@nist.gov

Ceramic processing and clean-room processing

Sintering and diffusion controlled processes

Bonnell, David

david.bonnell@nist.gov

Computer automation

Molecular-beam mass spectrometry

Thermodynamic modeling and measurement

Bouldin, Charles

charles.bouldin@nist.gov

X-ray absorption spectroscopy

Diffraction anomalous fine structure

GeSi heterojunction bipolar transistors

Burdette, Harold

harold.burdette@nist.gov

$\mathrm{X}$-ray optics

$\mathrm{X}$-ray diffraction imaging

Crystal growth

Instrumentation

Burton, Benjamin

Benjamin.burton@nist.gov

Calculated phase diagrams

Dielectric ceramics
Cellarosi, Mario

mario.cellarosi@nist.gov

Glass standards

Forensic data

Chan, Julia

Phase equilibria studies of dielectric oxides for wireless applications

Chuang, Tze-Jer

tze-jer.chuang@nist.gov

Creep/creep rupture

Fracture mechanics

Finite-element modeling

Lifetime predictions

Cline, James

james.cline@nist.gov

Standard reference materials

High-temperature $x$-ray diffraction

Microstructural effects in $x$-ray diffraction

Rietveld refinement of $\mathrm{x}$-ray diffraction data

Cockayne, Eric

eric.cockayne@nist.gov

First-principles computational studies of dielectric oxides

Cook, Lawrence

lawrence.cook@nist.gov

High-temperature chemistry

Phase equilibria

Dapkunas, Stanley

stanley.dapkunase@nist.gov

Databases

Ceramic coatings

Feldman, Albert

albert.Feldman@nist.gov

Thermal properties and modeling

Thin-film optical properties

Fischer, Daniel

daniel.fischer@nist.gov

$\mathrm{X}$-ray absorption fine structure

$\mathrm{X}$-ray scattering

Surface science 
Freiman, Stephen

stephen.freiman@nist.gov

Electronic ceramics

Mechanical properties

Fuller, Edwin

edwin.fuller@nist.gov

Influence of microstructure on fracture

Toughening mechanisms

Microstructural modeling and simulation

Gates, Richard

richard.gates@nist.gov

Tribo-chemistry

Surface chemical properties of ceramics

Gonzalez, Eduardo

Thermal measurement

Nano-scale sintering

Texture analysis

Hackley, Vincent

vince.hackley@nist.gov

Electrokinetic and electroacoustic measurement

Slurry rheology

Surface chemistry of powders

Harne, Mary

mary.harne@nist.gov

Phase equilibria data

Computerized data

Harris, Joyce

joyce.harris@nist.gov

Data acquisition

Digitization and data entry

Hastie, John

john.hastie@nist.gov

High-temperature chemistry

Phase equilibria thermochemistry

Molecular-beam mass spectrometry

Vapor deposition process control and modeling

Haugan, Timothy

Applications of phase equilibria data to

processing of high-temperature superconductors

Hockey, Bernard

bernard.hockey@nist.gov

Electron microscopy

High-temperature creep
Hsu, Stephen

stephen.hsu@nist.gov

Ceramic wear mechanisms

Engineered ceramic surfaces

Magnetic storage disk lubrication

Ives, Lewis

lewis.ives@nist.gov

Wear of materials

Transmission electron microscopy

Machining of ceramics

Jahanmir, Said

said.jahanmir@nist.gov

Ceramic manufacturing

Mechanisms of material removal

Mechanics of contacts

Effects of machining on mechanical properties

Kaiser, Debra

debra.kaiser@nist.gov

Ferroelectric oxide thin films

Physical properties and structures of high-

temperature superconductors

Karen, Vicky

vicky.karen@nist.gov

Crystallographic databases

Structural analysis

Kelly, James

james.kelly@nist.gov

Quantitative scanning electron microscopy

Image analysis

Microstructure analysis

Powder standards

Krause, Ralph

ralph.krause@nist.gov

Creep in flexure and tension

Fracture mechanics

Chemical thermodynamics

Long, Gabrielle

gabrielle.long@nist.gov

Small-angle $x$-ray and neutron scattering

Ceramic microstructure evolution as a function of processing

X-ray optics

Luecke, William

william.luecke@nist.gov

Creep/creep rupture

Mechanical test development

Ceramic membranes 
Lum, Lin-Sien

lin-sien.lum@nist.gov

Powder characterization

Instrumental analysis

McGuiggan, Patricia

patricia.mcguiggan@nist.gov

Microtribology

Surface force measurement

Minor, Dennis

dennis.minor@nist.gov

Analytical scanning electron microscopy of ceramics and particulates

Powder test sample preparation

Powder characterization

Munro, Ronald

ronald.munro@nist.gov

Materials properties of advanced ceramics

Data evaluation and validation

Analysis of data relations

Paul, Albert

albert.paul@nist.gov

Laser physics

Residual stress measurement

Pei, Patrick

patrick.pei@nist.gov

Spectroscopic and thermal characterization

Powders and green body characterization

Quinn, George

george.quinn@nist.gov

Mechanical property test standards

Standard reference materials

Robins, Lawrence

lawrence.robins@nist.gov

Defect identification and distribution

Cathodoluminescence imaging and spectroscopy

Photoluminescence spectroscopy

Raman spectroscopy

Roosen, Andrew

andrew.roosen@nist.gov

Microstructural modeling

Computer simulation

Rotter, Lawrence

Measurement of electro-optic coefficients

Photorefractive effect

Optical spectroscopy of thin films
Schenck, Peter

peter.schenck@nist.gov

Emission and laser speciroscopy

Thin-film deposition

Computer graphics and image analysis

Laboratory automation

Smith, Douglas

douglas.smith@nist.gov

Surface forces

Adhesion and friction

Instrumented Indentation

Spal, Richard

richard.spal@nist.gov

$\mathrm{X}$-ray optics

Diffraction physics

$\mathrm{X}$-ray scattering

Steiner, Bruce

High-resolution diffraction imaging

Defects in monolithic crystals and multiplayers

Non-linear optical processes

Vanderah, Terrell

terrell.vanderah@nist.gov

Solid-state chemistry

Phase equilibria of microwave dielectrics

Vaudin, Mark

mark.vaudin@nist.gov

Electron microscopy

Microscopy and diffraction studies of interfaces

Computer modeling of grain-boundary phenomena

Dielectric films

Wallace, Jay

jay.wallace@nist.gov

Mechanical test development

Ceramic coatings

Thermal analysis

Wang, Pu Sen

pu-wang@nist.gov

Solid-state nuclear magnetic resonance

Spectroscopic characterization

White, Grady

grady.white@nist.gov

Mechanical properties

Nondestructive evaluation

Stress measurements 
Woicik, Joseph

joseph.woicik@nist.gov

UV photoemission

$\mathrm{X}$-ray standing waves

Surface and interface science

Wong-Ng, Winnie

winnie.wong-ng@nist.gov

$\mathrm{X}$-ray crystallography and reference patterns

Phase equilibria/crystal chemistry of high-Tc

superconductors

\section{GUEST SCIENTISTS AND GRADUATE STUDENTS}

Armstrong, Nicholas

University of Technology, Sydney Australia

Attota, Ravikiran

National University of Singapore

Bai, Mingwur

Tohoku University

Boukari, Hacene

University of Maryland

Cedeno, Christina

American Ceramic Society

Dillingham, Jermey

University of Maryland

Early, James

Consultant

Fang, Hsu-Wei

University of Maryland

Farabaugh, Edward

American Ceramic Society

Fu, Zugen

State Univ. of N.Y. at Stony Brook

Haller, Wolfgang

Consultant

Hayward, Evans

American Ceramic Society

Hryniewicz, Piotr

University of Delaware

Ilavsky, Jan

University of Maryland
Jemian, Peter

University of Illinois at Urbana/Champaign

Jillavenkatesa, Ajitkumar

Alfred University

Kalin, Mitjan

University of Ljubljana

Kieffer, John

University of Illinois

Kulkarni, Anand

State University of New York

Kweon, Hyun-kyu

Tohoku University

Larsen-Basse, Jorn

National Science Foundation

Levin, Igor

University of Maryland

Li, $\mathrm{Na}$

Beijing U. of Aeronautics \& Astronautics

Lindsey, Tammy

Appalachian State University

Ling, Yin

Tianjin University

McMurdie, Howard

Joint Center for Powder Diffraction Studies

Mighell, Alan

Consultant

Nuffer, Juergen

University of Tech. Darmstadt

Ondik, Helen

Consultant

Ozoon, Takuya

Tokyo Institute of Technology

Park, Youngsoo

University of Illinois

Piermarini, Gasper

University of Maryland

Quinn, Janet

American Dental Association 
Ritter, Joseph

Consultant

Roberts, Ellis

Consultant

Roth, Robert

Viper Group

Sambasivan, Sharadha

Brookhaven National Laboratory

Shen, Ming

University of Illinois

Swab, Jeffrey

Army Research Laboratory

Swanson, Nils

American Ceramic Society

Turchinskaya, Marina

Consultant

Winburn, Ryan

University of North Dakota

Wolfenstine, Jeffrey

U S. Army Research Laboratory

Yeager, Glenn

Trak Ceramics, Inc.

Yin, Zhanfeng

University of Maryland 


\section{Organizational Charts}

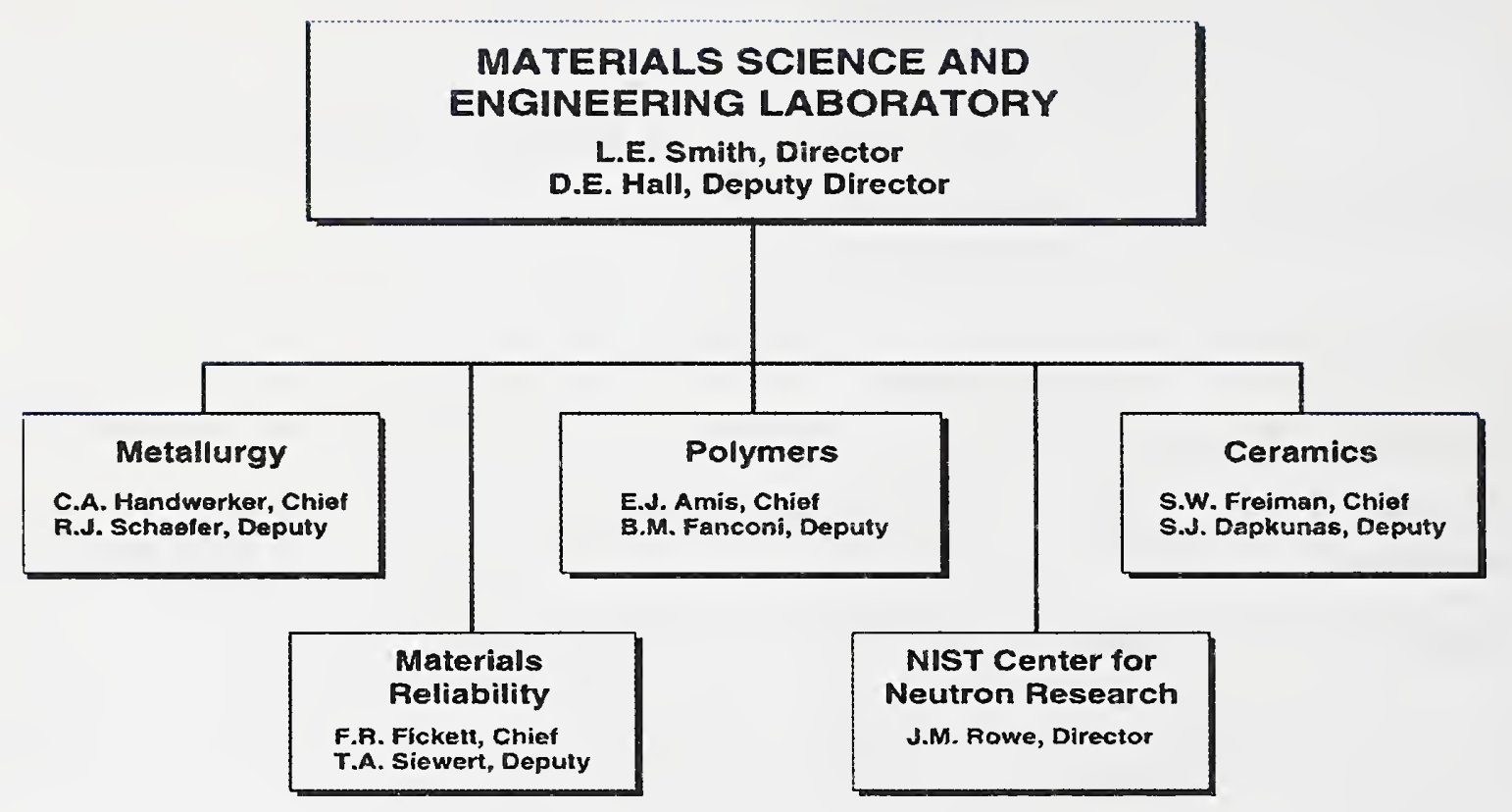

\section{National Institute of Standards and Technology}

\section{Organizational Chart}

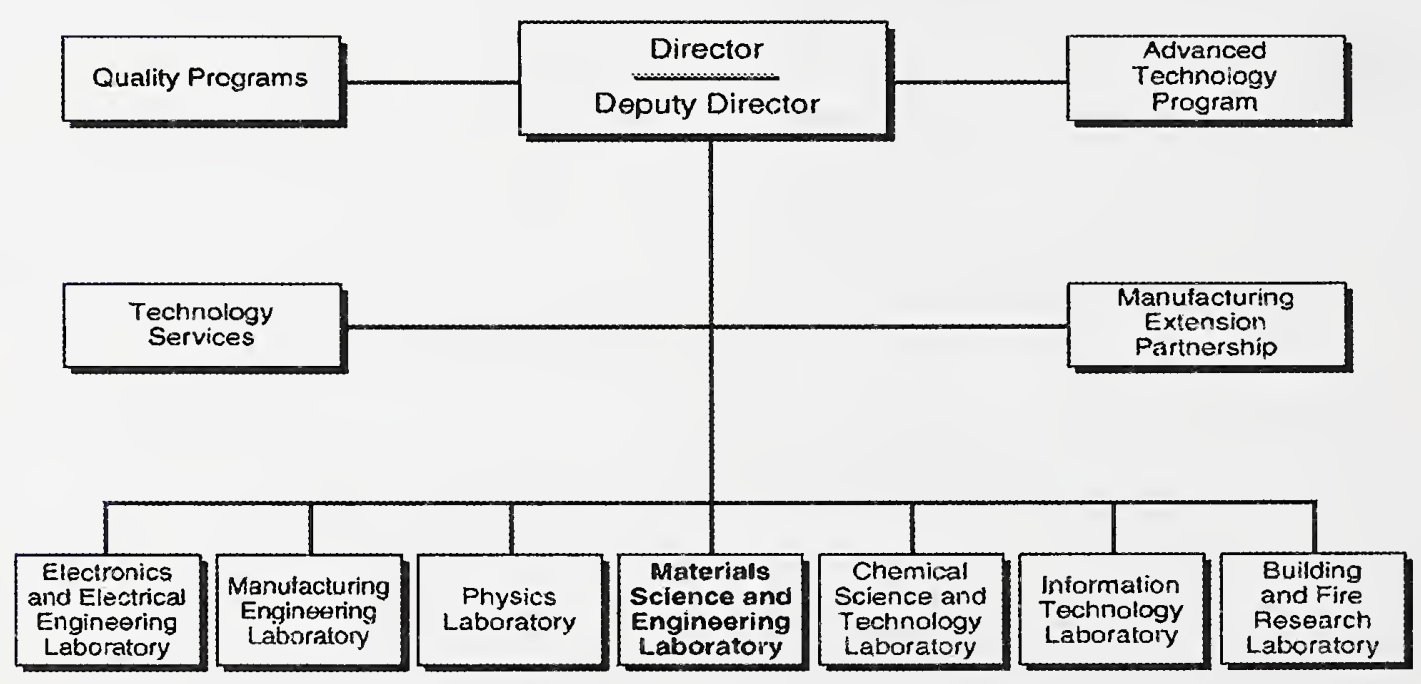





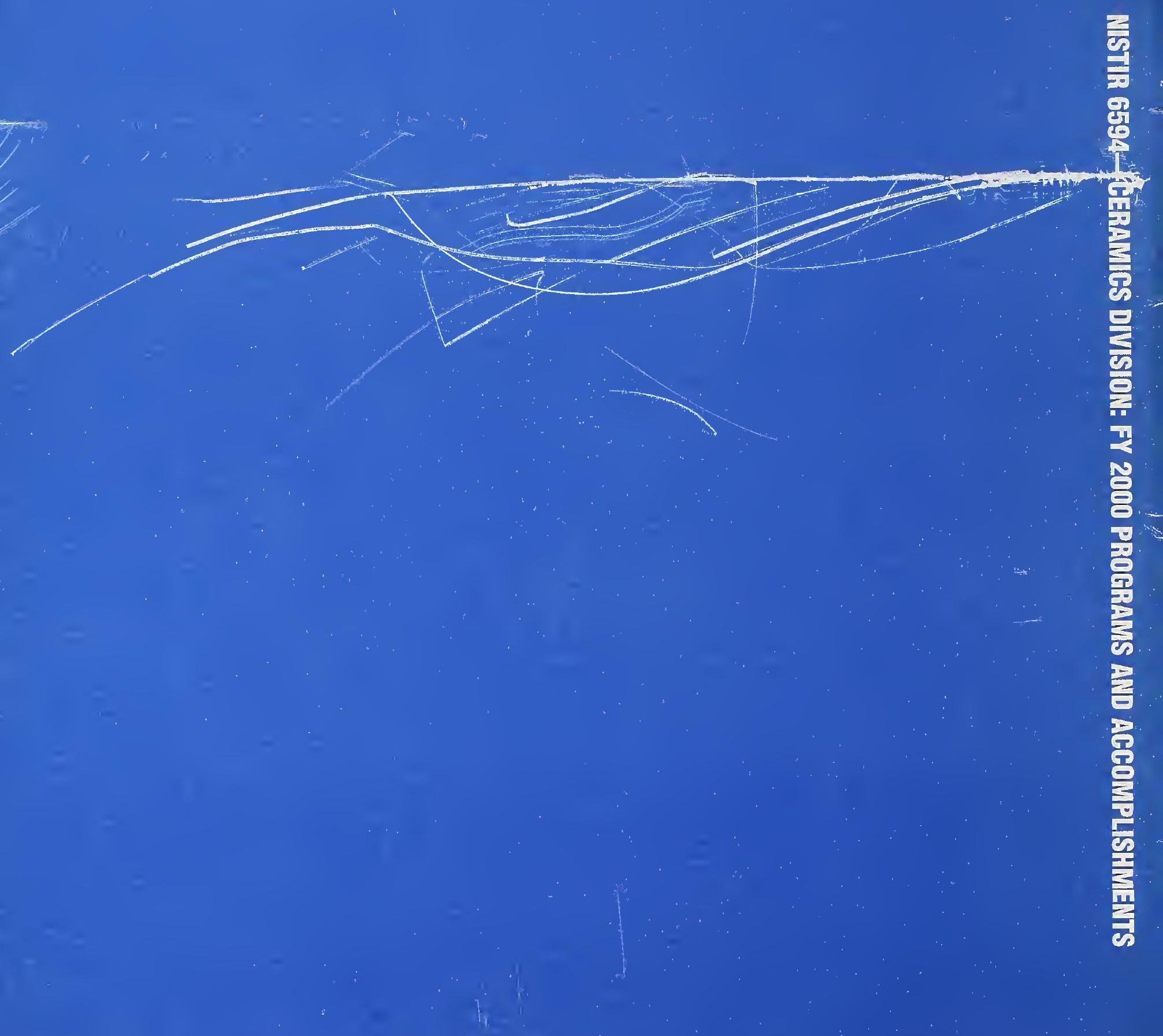

